\title{
Large Eddy Simulation of Rayleigh-Taylor Instability Using the Arbitrary Lagrangian-Eulerian Method
}

Rebecca Mattson Darlington

December 1, 1999

Lawrence

Livermore

National

Laboratory 



\section{DISCLAIMER}

This document was prepared as an account of work sponsored by an agency of the United States Government. Neither the United States Government nor the University of California nor any of their employees, makes any warranty, express or implied, or assumes any legal liability or responsibility for the accuracy, completeness, or usefulness of any information, apparatus, product, or process disclosed, or represents that its use would not infringe privately owned rights. Reference herein to any specific commercial product, process, or service by trade name, trademark, manufacturer, or otherwise, does not necessarily constitute or imply its endorsement, recommendation, or favoring by the United States Government or the University of California. The views and opinions of authors expressed herein do not necessarily state or reflect those of the United States Government or the University of California, and shall not be used for advertising or product endorsement purposes.

Work performed under the auspices of the U. S. Department of Energy by the University of California Lawrence Livermore National Laboratory under Contract W-7405-Eng-48.

This report has been reproduced directly from the best available copy.

Available to DOE and DOE contractors from the Office of Scientific and Technical Information

P.O. Box 62, Oak Ridge, TN 37831

Prices available from (423) 576-8401

http://apollo.osti.gov/bridge/

Available to the public from the National Technical Information Service

U.S. Department of Commerce 5285 Port Royal Rd., Springfield, VA 22161 http://www.ntis.gov/

OR

Lawrence Livermore National Laboratory

Technical Information Department's Digital Library

http://www.llnl.gov/tid/Library.html 



\section{Large Eddy Simulation of Rayleigh-Taylor Instability Using the Arbitrary Lagrangian-Eulerian Method}

Rebecca Mattson Darlington

PhD Dissertation

Manuscript date: December 1, 1999

LAWRENCE LIVERMORE NATIONAL LABORATORY University of California $\cdot$ Livermore, California 94551 





\title{
Large Eddy Simulation of Rayleigh- Taylor Instability using the Arbitrary Lagrangian-Eulerian Method
}

\author{
by \\ REBECCA MATTSON DARLINGTON \\ B.S. (University of New Hampshire) 1993 \\ M.S. (University of California, Davis) 1996
}

DISSERTATION

Submitted in partial satisfaction of the requirements for the degree of

DOCTOR OF PHILOSOPHY

in

ENGINEERING-APPLIED SCIENCE

in the

OFFICE OF GRADUATE STUDIES

of the

UNIVERSITY OF CALIFORNIA

DAVIS

Approved:

Committee in Charge

1999 
Rebecca Mattson Darlington

December 1999

Engincering-Applied Science

\title{
Large Eddy Simulation of Rayleigh- Taylor Instability using the Arbitrary Lagrangian-Eulerian Method
}

\begin{abstract}
$\underline{\text { Abstract }}$
This research addresses the application of a large eddy simulation (LES) to Arbitrary Lagrangian Eulerian (ALE) simulations of Rayleigh-Taylor instability. First, ALE simulations of simplificd Raylcigh-Taylor instability are studied. The advantages of ALE over Eulerian simulations are shown. Next, the behavior of the LES is examined in a more complicated ALE simulation of Rayleigh-Taylor instability. The effects of eddy viscosity and stochastic backscatter are examined. The LES is also coupled with ALE to increase grid resolution in areas where it is needed. Finally, the methods studied above are applied to two sets of experimental simulations. In these simulations, ALE allows the mesh to follow expanding experimental targets, while LES can be used to mimic the effect of unresolved instability modes.
\end{abstract}




\section{Acknowledgments}

I wish to thank the many people who made this dissertation possible. First of all, I would like to thank my two thesis advisors: Dr. Tom McAbee for the thesis project idea and guidance in how to accomplish it, and Dr. Garry Rodrigue for assistance with the computational details of the project and writing. Without the continual advice of both my advisors, I would never have accomplished this work.

Many former UC Davis students also offered advice and encouragement indispensable to my success. Paul Amala, Colin Aro, Evi Dube, and Rose McCallen all helped me through the technical details of my research, as well as the process of getting through school.

Guy Dimonte and Marilyn Schneider provided information on the Linear Electric Motor experiments. Kim Budil and Steve Weber provided information on laser driven instability experiments on NOVA.

Finally I would like to thank all those who created the ARES code, in particular Brian Pudliner and Greg Greenman. They helped me understand the details of the code I needed to accomplish this research. 
Abstract $\ldots \ldots \ldots \ldots \ldots \ldots \ldots \ldots \ldots \ldots \ldots \ldots \ldots \ldots \ldots \ldots \ldots \ldots \ldots \ldots$ ii

Acknowledgments ..................................iii

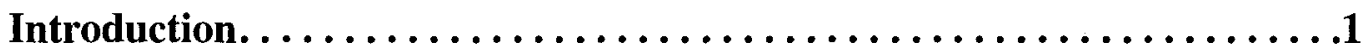

ALE Simulation of Rayleigh-Taylor Instability. . . . . . . . . . . . .

1.0 Motivation for ALE $\ldots \ldots \ldots \ldots \ldots \ldots \ldots \ldots$

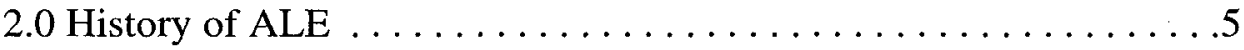

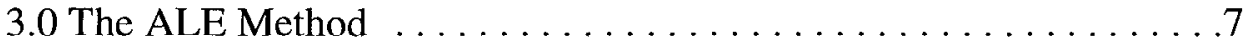

3.1 The Lagrangian phase $\ldots \ldots \ldots \ldots \ldots \ldots \ldots$

3.2 The Eulerian phase $\ldots \ldots \ldots \ldots \ldots \ldots$

4.0 ALE Variations . . . . . . . . . . . . . . . . . . . . 10

5.0 Single mode Rayleigh-Taylor instability test problem . . . . . . . .11

5.1 Comparing ALE to Eulerian mesh motion . . . . . . . . 14

5.2 The effect of the Lagrangian step on Rayleigh-Taylor growth 18

5.3 The effect of grid relaxation on Rayleigh-Taylor growth . . .19

5.4 The effect of remap on Rayleigh-Taylor growth . . . . . .26

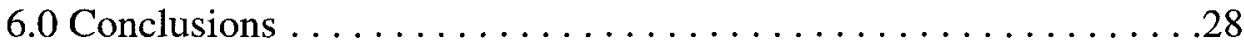

Large Eddy Simulation and ALE .....................

7.0 Motivation for $\operatorname{LES} \ldots \ldots \ldots \ldots \ldots \ldots \ldots \ldots \ldots \ldots \ldots \ldots \ldots \ldots$

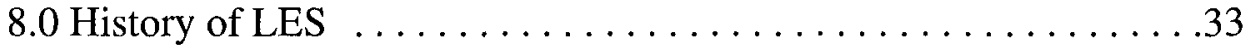

9.0 Equations of motion $\ldots \ldots \ldots \ldots \ldots \ldots \ldots \ldots \ldots \ldots \ldots \ldots \ldots$

10.0 Cloutman model $\ldots \ldots \ldots \ldots \ldots \ldots \ldots \ldots \ldots \ldots \ldots \ldots . \ldots . \ldots \ldots$

10.1 New equations of motion $\ldots \ldots \ldots \ldots \ldots \ldots \ldots \ldots$

10.2 Stochastic backscatter . . . . . . . . . . . . . 47

11.0 Rayleigh-Taylor instability test problem . . . . . . . . . . .49

12.0 Cloutman model effects on the test problem $\ldots \ldots \ldots \ldots \ldots . \ldots 5$

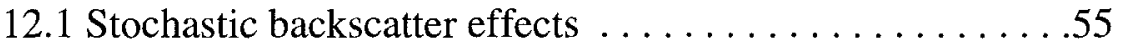

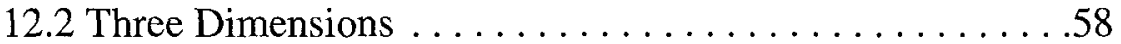

13.0 ALE mesh motion and LES . . . . . . . . . . . . 60

13.1 Subgrid scale kinetic energy as a grid relaxation weight . .65

14.0 ALE vs. Eulerian LES . . . . . . . . . . . . . . . . 68

14.1 Convergence study ................70

15.0 Conclusions $\ldots \ldots \ldots \ldots \ldots \ldots \ldots \ldots \ldots \ldots \ldots \ldots \ldots \ldots \ldots \ldots \ldots$

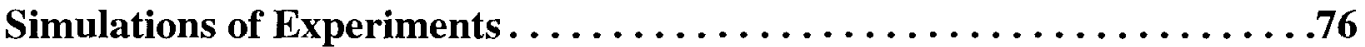

16.0 Simulation of experiments with the Linear Electric Motor . . . . .76

16.1 Simulation details $\ldots \ldots \ldots \ldots \ldots \ldots \ldots 77$ 
16.2 Simulation results $\ldots \ldots \ldots \ldots \ldots \ldots \ldots \ldots . \ldots . \ldots 2$

16.3 The effect of LES in 2 and 3 dimensions . . . . . . . 85

16.4 Atwood number dependence . . . . . . . . . . . . .90

16.5 Simulation observations $\ldots \ldots \ldots \ldots \ldots \ldots \ldots .93$

17.0 Simulation of laser driven Rayleigh-Taylor instability experiments 93

17.1 Beat modes ........................... .95

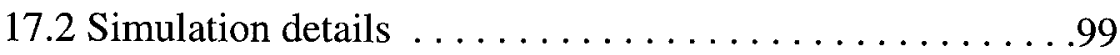

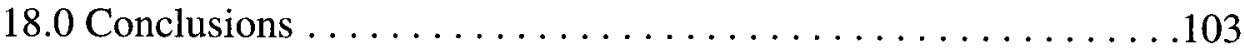

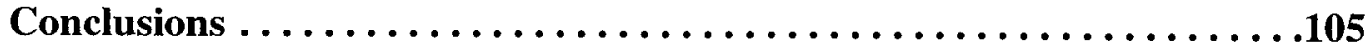

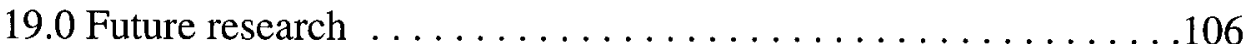

Appendix A: Rayleigh-Taylor Instability $\ldots \ldots \ldots \ldots \ldots \ldots \ldots \ldots \ldots . \ldots 107$

1.0 Linear stability analysis $\ldots \ldots \ldots \ldots \ldots \ldots \ldots \ldots . \ldots \ldots$

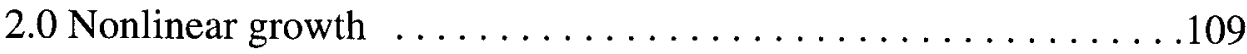

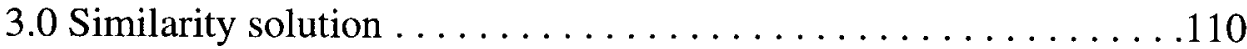

Appendix B: Solving the Equations of Motion ................111

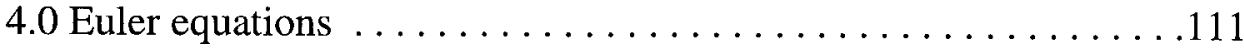

4.1 Conservation of mass . . . . . . . . . . . . . . . . 112

4.2 Conservation of momentum ...............112

4.3 Conservation of internal energy $\ldots \ldots \ldots \ldots \ldots \ldots .114$

4.4 Predictor step .............................

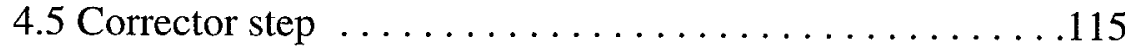

5.0 Equations of motion for the Cloutman model . . . . . . . . . . 116

5.1 Solving the equations of motion ..................119

5.2 Stochastic backscatter . . . . . . . . . . . . . . . . 124

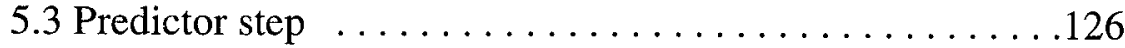

5.4 Corrector step . . . . . . . . . . . . . . . . . 127

Appendix C: Grid Relaxation $\ldots \ldots \ldots \ldots \ldots \ldots \ldots \ldots \ldots \ldots \ldots . .130$

6.0 Motivation for grid relaxation . . . . . . . . . . . . . . . 130

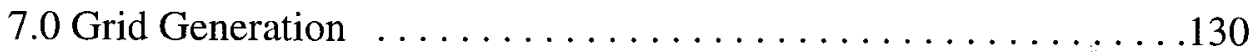

References ....................................136 


\section{Introduction}

The purpose of this thesis is to combine the methods of Arbitrary Lagrangian-Eulerian (ALE) simulation with large eddy simulation (LES), and to explore the advantages of the combination. Rayleigh-Taylor instability simulation has been studied extensively. Simulations have been compared with mathematical theories of Rayleigh-Taylor growth and experimental observations. The subject of the research ranges from astrophysics to inertial confinement fusion (ICF). So far, studies have been carried out mainly with Eulerian methods. This dissertation finds that in Rayleigh-Taylor simulations of experi- . ments such as ICF, ALE and LES are both important. ALE allows the simulation to follow the changing shape of the target, while LES approximates missing features.

Many Eulerian simulations of Rayleigh-Taylor instability have been published by Youngs ([46][72][73][74][75]) and Glimm et al. ([23][27][28][29][45]). Youngs employed an interface reconstruction scheme to track the movement of the fluid interface across an Eulerian mesh. The interface was reconstructed from the volume fractions of various materials in neighboring zones. He simulated experiments conducted by Read [54] and Linden et al. [46]. He studied in particular the role of Rayleigh-Taylor instability in small scale mixing, and included a turbulence model (another name for LES) in his 2 and 3 dimensional compressible simulations.

The method employed by Glimm et al. is a front tracking across an Eulerian mesh. The front between the two fluids moves in a Lagrangian manner past the fixed grid. The front serves as an extra degree of freedom, in that tracking it involves solving elliptic PDEs at each time step. They developed statistical models for the processes of bubble growth and merger. They also showed that the bubble growth rate $\alpha$ is a renormalization 
fixed point, a universal constant.

ALE was used to simulate Rayleigh-Taylor instability by Alon, Freed, Hecht, Shvarts, and others ([1][21][31][52]). They employed an interface tracking scheme at the interface, and developed a two-phase flow model of mixing. This is a typc of turbulence model, for simulations in which neighboring fluids in a grid zone have separate velocities. The two phase flow model approximates the drag, acceleration, mass, and length scale changes caused by the interaction of the two fluids. Unlike the LES in this study, filtering and closure approximations were not used. Simulations were conducted at various Atwood numbers, and 3 dimensional simulations of ICF pellets were conducted as well.

This research addresses three questions: first, how does ALE mesh motion effect the simulation of Rayleigh-Taylor instability? Secondly, how does LES work with ALE? Mesh motion alters the definition of resolved and unresolved scales, and makes filtering depend on time and space. Finally, how can ALE mesh motion and LES be used together to improve simulations of experiments?

To answer the questions, the simplest case was examined first. The growth of a resolved, single mode initial perturbation was studied with ALE mesh motion. The effect of varying the mesh motion was explored. When compared with higher resolution simulations, ALE simulations more accurately captured the instability growth and energy balance than Eulerian simulations.

Next, the behavior of the LES was studied in a more complicated ALE simulation with a perturbation composed of several resolved modes. A more complicated interface evolved due to mode coupling. The performance of the LES was similar with ALE and 
Eulerian mesh motion. However, the ALE mesh motion could be coupled with the LES to increase resolution where needed.

Simple simulations show the behavior of LES in an ALE simulation, while more complicated simulations of experiments show where ALE and LES are most useful. Stochastic backscatter was combined with LES to represent unresolved modes in the initial perturbation. The ALE method was important in following the changing shape of an experimental target. These simulations illustrate the importance of both ALE mesh motion and LES in simulation of Rayleigh-Taylor instability experiments. 


\section{ALE Simulation of Rayleigh-Taylor Instability}

This chapter investigates the use of the Arbitrary Lagrangian-Eulerian (ALE) method in simulations of Rayleigh-Taylor instability. The purpose is to determine the best way to use ALE to improve simulations.

\subsection{Motivation for ALE}

In computational simulations, a Lagrangian grid moves with the material being simulated. An Eulerian grid remains stationary while the material moves past it. An ALE method combines Lagrangian and Eulerian simulation techniques.

Lagrangian simulations describe material interfaces and domain boundaries very well, since the grid follows the changing shape of the material. There is no need to deal with mixed zones, which contain more than one material. Grid points cluster in areas of large gradients, such as shocks. Fewer grid points are needed elsewhere, so the method can be computationally faster than the Eulerian method. But the grid cannot follow all material motion. A simulation cannot continue if mesh lines cross and create singular points. Also, derivatives become increasingly inaccurate as the zones become less uniform.

An Eulerian mesh can describe any flow, no matter how twisted. Since the Eulerian mesh doesn't follow the flow, a uniform orthogonal mesh at the beginning of the problem will still be uniform and orthogonal later on. This makes approximating derivatives much simpler. But Eulerian grids contain mixed zones, which require extra time and storage. Extra grid points are needed to simulate structures that move across the grid. Otherwise information can be lost between grid lines. This makes Eulerian problems 
computationally slower. Eulerian simulations also suffer higher numerical dissipation.

ALE has several advantages over pure Lagrangian or pure Eulerian simulations. It can adapt to local flow conditions, moving in a Lagrangian fashion in areas where fluid motion is less, and remaining fixed in areas where fluid motion is greater. In between, the mesh follows the flow part way, but not enough to tangle. The disadvantage of ALE is greater advection error relative to Lagrangian simulations, but smaller advection error relative to Eulerian simulations. Also, greater computational effort is needed over Lagrangian or Eulerian simulations.

Eulerian mesh

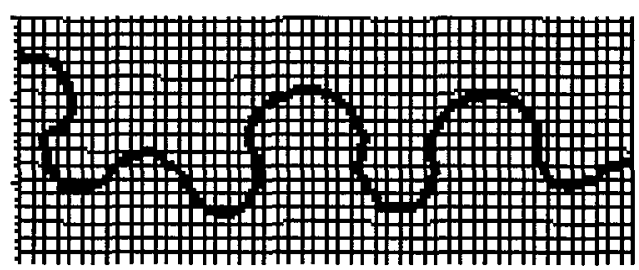

ALE mesh

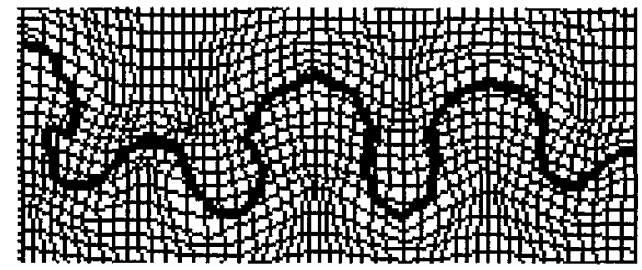

Lagrangian mesh

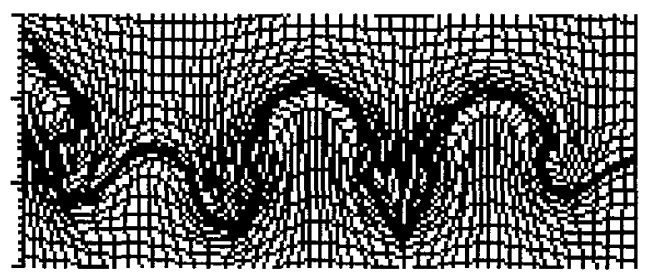

Figure 1: Mesh motion

\subsection{History of ALE}

In the 1960 's methods of simulating material motion with a moving mesh were first 
developed. Moving the mesh along with the material being modeled is useful for some flows. Wilkins [69] created an early Lagrangian code to describe elastic-plastic flows. Lagrangian codes are still used to simulate materials that do not deform enough to cause mesh lines to cross, but this is rarely true of fluids.

Several combinations of Eulerian and Lagrangian meshes were attempted. Frank and Lazarus [20] tried a mesh that was Eulerian in the vertical direction and Lagrangian in the horizontal direction. Noh [51] tried a mesh that was Eulerian in some portions and Lagrangian in others. Some Lagrangian codes were stopped in order to untangle the mesh and then resumed.

Systematic ways to combine the Eulerian and Lagrangian concepts of mesh motion were developed to alleviate the need for stopping a simulation in progress. When Lagrangian simulations were stopped in mid flow to untangle the mesh, inaccuracies occurred in adjusting the material to fit the new mesh. A method of advecting material past grid lines was clearly needed, to redistribute the physical quantities on the new mesh without altering the physics of the flow. Accurate second order advection schemes were developed by Roe [55], and Van Leer [67].

Automatic ways to adjust the grid in mid flow eliminated the need for stopping the simulation to undo grid tangles. The mesh is moved by employing a transformation between a uniform orthogonal mesh and the nonuniform, nonorthogonal mesh arising from the simulation. An algebraic transformation can be used, or an elliptic partial differential equation [17].

In 1972 the ALE method was described by Hirt, Amsden, and Cook [33]. Many ALE codes have followed, using finite element and finite volume methods to approxi- 
mate the equations of motion.

An ALE simulation adds an extra velocity to the equations of motion in the form of

mesh velocity. The Lagrangian time derivative $\frac{d}{d t}$ is related to the Eulerian time derivative $\frac{\partial}{\partial t}$ by

$$
\frac{d}{d t}=\frac{\partial}{\partial t}+u \cdot \nabla
$$

where $u$ is the fluid velocity. If the mesh velocity is $v$, the ALE time derivative is related to the Lagrangian time derivative by the following relationship:

$$
\frac{d}{d t}=\frac{\partial}{\partial t}+(u-v) \cdot \nabla
$$

This creates a set of ALE equations of motion similar to the Eulerian equations of motion.

However, in the method used in this study the grid velocity is not prespecified. Instead the grid must be tested at each time step, to determine if it is in danger of tangling. Nodes are tested individually, so some may move while their neighbors remain fixed. This makes it impossible to solve the ALE time derivatives in EQ 2. Instead, the motion of the mesh is calculated at each time step, separately from the solution of the equations of motion.

\subsection{The ALE Method}

The ALE method in this study consists of two phases: The Lagrangian phase and the Eulerian phase. In the Lagrangian phase, the Lagrangian equations of motion EQ 3 - EQ 
5 are solved with the mesh following the fluid. Then, in the Eulerian phase, the grid is relaxed to a new position and the material is advected between zones in response to their new locations.

\subsection{The Lagrangian phase}

In the Lagrangian phase of the ALE method, the Lagrangian equations of motion are solved using a second order accurate predictor-corrector scheme. The inviscid equations of motion in Lagrangian coordinates are:

$$
\begin{gathered}
\frac{1}{\rho} \frac{d \rho}{d t}=-\nabla \bullet \boldsymbol{u} \\
\rho \frac{d \boldsymbol{u}}{d t}=-\nabla p+\rho g \\
\rho \frac{d e}{d t}=-p \nabla \bullet \boldsymbol{u}
\end{gathered}
$$

where $\frac{d}{d t}=\frac{\partial}{\partial t}+u \bullet \nabla$ is the Lagrangian derivative, $\rho$ is the density, $\boldsymbol{u}$ is the velocity vector, $g$ is the gravitational acceleration, $e$ is the internal energy, and $p$ is the pressure, given in this case by the ideal gas law $p=(\gamma-1) \rho e$ where $\gamma=5 / 3$. Details of the solution to the equations of motion are found in Appendix B, Section 4.0 on page 111.

\subsection{The Eulerian phase}

In the Lagrangian step, the mesh moved with the fluid, but now parts of it may need to be moved back, to reduce the distortion of the grid. This preserves accuracy and allows the program to continue to follow a complicated flow in the next time step.

First, a new grid is chosen, using information from the old grid, to represent the flow 
more accurately in the next Lagrangian step. This is the grid relaxation step. Then the physical variables must be redefined in response to the new position of grid points in the fluid. This is the remap step, which employs various advection approximations. These two processes are referred to as the Eulerian step.

Although the fluid does not advance in time during the Eulerian step, approximations are made when the physical variables are defined on the new grid. Therefore, mass momentum and energy may change during the Eulerian step.

\subsubsection{Grid Relaxation}

A new grid is found by an equipotential relaxation method[63]. A description of grid relaxation can be found in Appendix $\mathrm{C}$.

\subsubsection{Remap}

Once the new grid is chosen, mass, momentum, and energy need to be redistributed into the new zones. Using $\phi$ to represent any zone centered quantity and $V$ to represent the zonal volume, a one dimensional remap is shown in Fig. 2 .

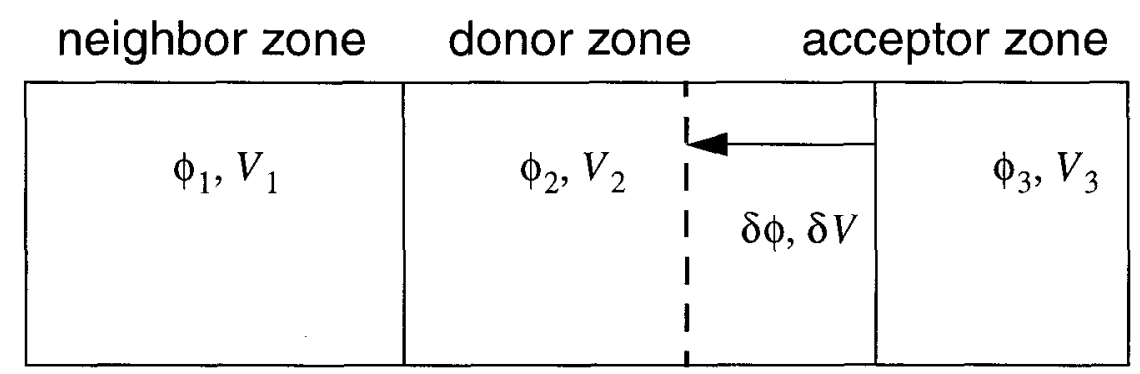

Figure 2: Remap

The new quantities (denoted by primes) are calculated as follows: 


$$
V_{3}^{\prime} \cdot \phi_{3}^{\prime}=V_{3} \cdot \rho_{3}+\delta V \cdot \delta \varphi \text { and } V_{2}^{\prime} \cdot \phi_{2}^{\prime}=V_{2} \cdot \rho_{2}-\delta V \cdot \delta \varphi
$$

The value of $\delta \phi$ is found by a second order approximation of the derivative in

$$
\delta \phi=\phi_{2}+\left.\frac{\partial \phi}{\partial x}\right|_{x_{0}}\left(x-x_{0}\right)
$$

where $x_{0}$ is the physical center of $\delta V$. The value of $\delta \phi$ is subject to monotonicity constraints. That is, the process of moving material from one grid zone to another must not create any new maxima or minima in the physical properties of the flow. Monotonicity constraints reduce the order of the approximation to first locally where needed.

Two second order advection schemes or slope limiters [42] are used in this study: the "Superbee" limiter of Roe [55] and the van Leer [67] slope limiter. We also consider a scheme in which advection is a first order approximation everywhere.

\subsection{ALE Variations}

In order to determine how ALE can best be used to improve Rayleigh-Taylor instability simulations, different ALE parameters were modified. As can be seen from previous sections, there are many possible modifications to ALE mesh motion. Table 1 lists the parameters that will be discussed in later sections. 
Table 1: ALE variations

\begin{tabular}{|c|c|c|}
\hline ALE Variation & Default & Range \\
\hline \multicolumn{3}{|l|}{ Lagrangian Step Variations } \\
\hline \# Lagrange steps between remap steps & 1 & $1-\infty$ \\
\hline \multicolumn{3}{|l|}{ Grid Relaxation Variations } \\
\hline angle test for grid relaxation & $60^{\circ}$ & $0-90^{\circ}$ \\
\hline area test for grid relaxation & 0.05 & $0.0-0.25$ \\
\hline \# iterations in relaxation & 1 & $1-\infty$ \\
\hline $\begin{array}{l}\text { multiplier for maximum distance } \\
\text { relaxer can move }\end{array}$ & 4.0 & $1-\infty$ \\
\hline material relaxation weight & 1.0 & $1-\infty$ \\
\hline $\begin{array}{l}\text { \# times relaxation weights are averaged } \\
\text { with neighboring weights }\end{array}$ & 1 & $1-\infty$ \\
\hline $\begin{array}{l}\text { \# times replace local weights with largest } \\
\text { of nearest neighbors }\end{array}$ & 1 & $1-\infty$ \\
\hline \multicolumn{3}{|l|}{ Advection Variations } \\
\hline Advection Method: & 1 & 1,2, or 3 \\
\hline \multicolumn{3}{|l|}{ 1. $x$ then $y$ this time step, $y$ then $x$ next time step } \\
\hline \multicolumn{3}{|l|}{ 2. half $x$, full $y$, finish $x$} \\
\hline \multicolumn{3}{|l|}{ 3. zone by zone } \\
\hline Advection Scheme: & 2 & 1,2, or 3 \\
\hline \multicolumn{3}{|l|}{ 1. Roe Superbee advectiun } \\
\hline \multicolumn{3}{|l|}{$\begin{array}{l}\text { 2. Roe Superbee for momentum and Van Leer for scalar } \\
\text { advection }\end{array}$} \\
\hline 3. first order advection & & \\
\hline
\end{tabular}

\subsection{Single mode Rayleigh-Taylor instability test problem}

The following test problem was used to determine how ALE mesh motion effects the growth of a single mode Rayleigh-Taylor instability. An ideal gas of density 2.0 was placed above an ideal gas of density 1.0 in a square domain $0 \leq x, y \leq 10$. A uniform downward gravitational field of magnitude 1.0 was assumed. Boundaries were reflecting. The fluid interface had the following initial shape: 


$$
y=5.0-A \sin \left(\frac{2 \pi x}{\lambda}+\frac{\pi}{2}\right)
$$

where the wavelength $\lambda$ was taken to be 2.0 (see Fig. 9 ). Initially the pressure gradient balanced gravity in the $y$ direction.

$$
\frac{\partial p}{\partial y}=(\gamma-1) \rho \frac{\partial e}{\partial y}=-\rho g
$$

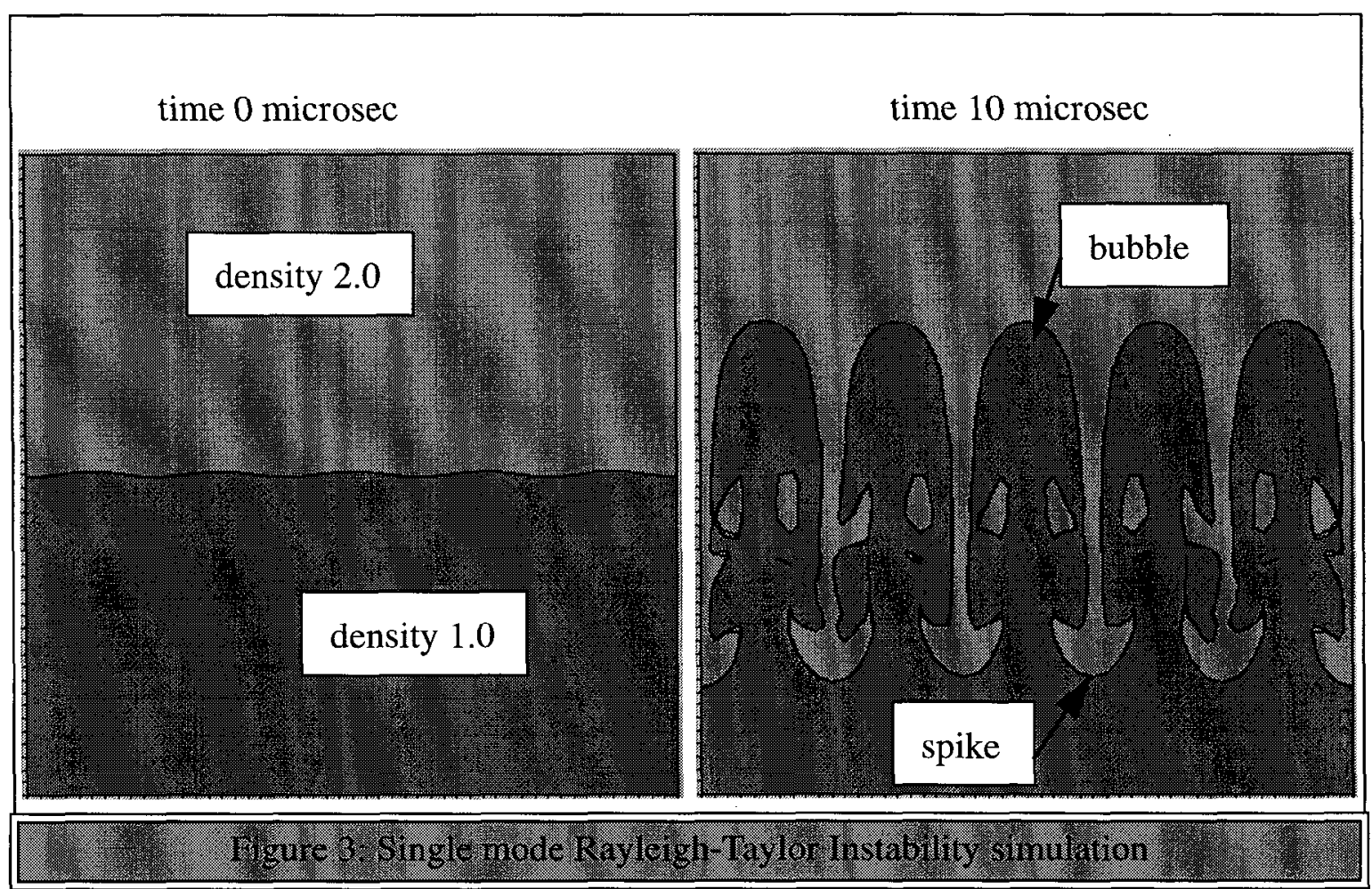

The default values are shown in Table 1.

The Rayleigh-Taylor bubble and spike must be defined and tracked over time to determine their growth rates. An interface reconstruction method was used [64]. The slope of the fluid interface was determined from the amount of each material in each zone and its neighboring zones. The intercept of the material interface with the edge of the zone was not calculated however.

In order to determine the location of the bubble and spike fronts in the presence of 
ALE mesh motion over time, a vertical scale was set up initially. The scale was the same height as the domain, and was divided into as many bins as there are grid zones in the vertical direction. The vertical position of each zone placed it in a particular bin on the vertical scale (see Fig. 4 ). The mass of each material in each zone was added to the total for that bin, so mass fractions could be defined along the vertical scale. This way, the location of the bubble and spike fronts were located according to a vertical scale which did not move as the mesh moved.

The mass fraction of both materials was considered in each bin, starting from the top. The actual location of the interface was defined as the center of the bin where the upper material fell below $95 \%$, and the spike front was defined as the center of the bin where the upper material fell below 5\%. Consequently, for low resolution simulations, plots of the interface position over time had a stair step appearance. Smooth plots of the instability growth were created by fourth order polynomial interpolation.

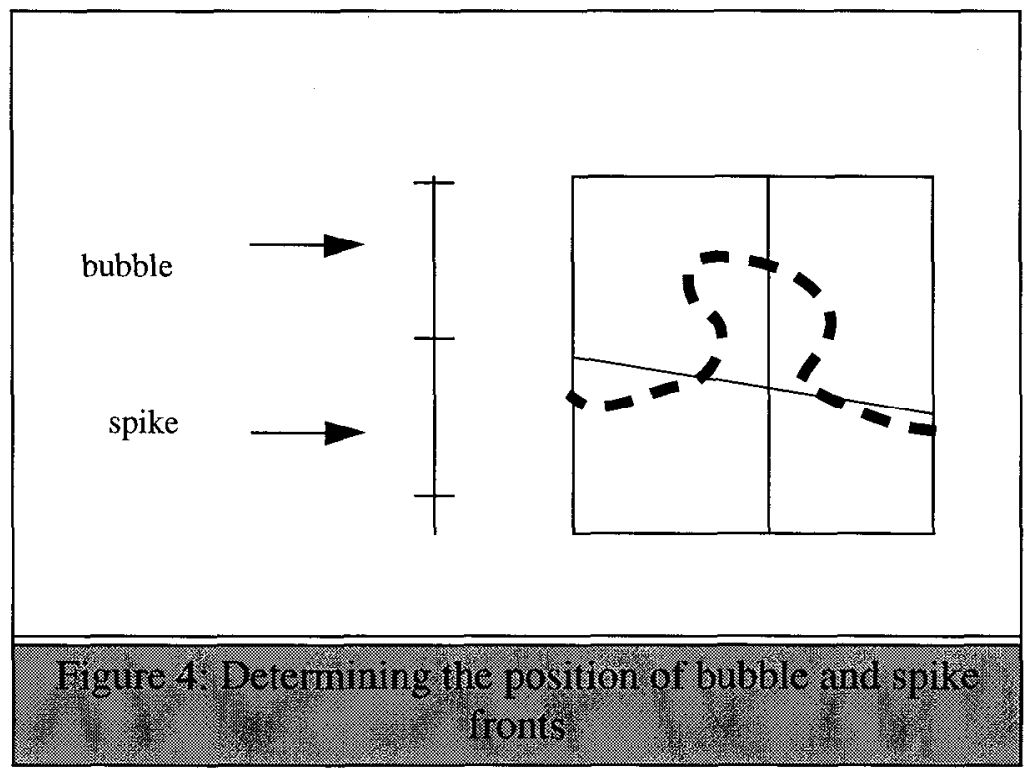

The mesh resolution had a great effect on the Rayleigh-Taylor growth rate. It took at 
least 4 zones per wavelength to resolve a mode. However, the growth rate approached a uniform value above a resolution of $50 \times 50$ zones (see Fig. 5 ).

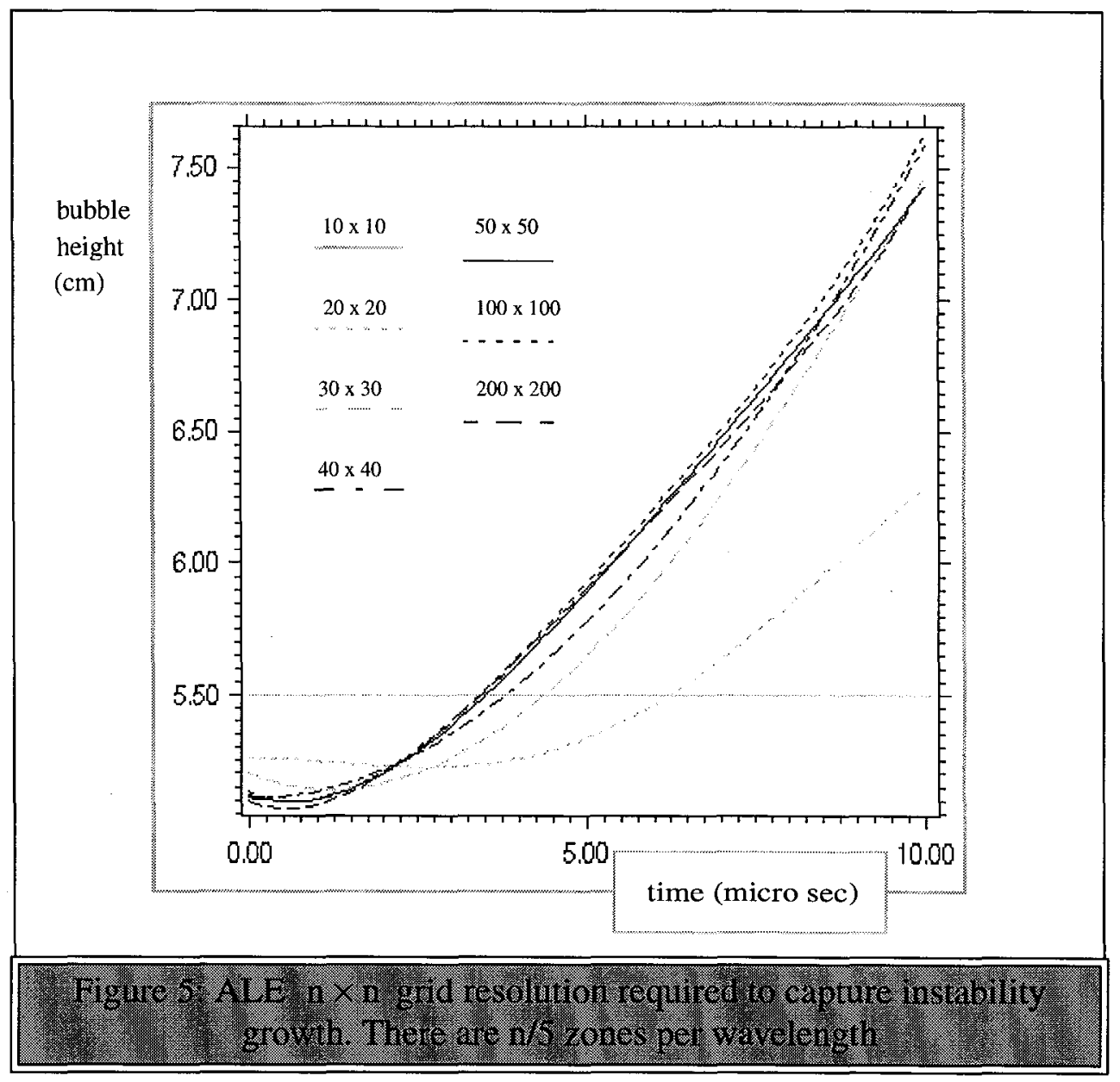

\subsection{Comparing ALE to Eulerian mesh motion}

The ALE method in this study can be modified to create a two step Eulerian simulation. The Lagrangian step remains unchanged, but in the remap step the mesh is returned to its original position. In all calculations, the "reference" solution was obtained by computing the Rayleigh-Taylor problem using this two-step Eulcrian method on a $200 \times 200$ grid.

Rayleigh-Taylor growth in ALE simulations was not dramatically different from Eulerian simulations. However, early in the simulation ALE mesh motion allowed 
slightly faster instability growth than Eulerian, which made it more accurate in comparison to the higher resolution Eulerian simulation.

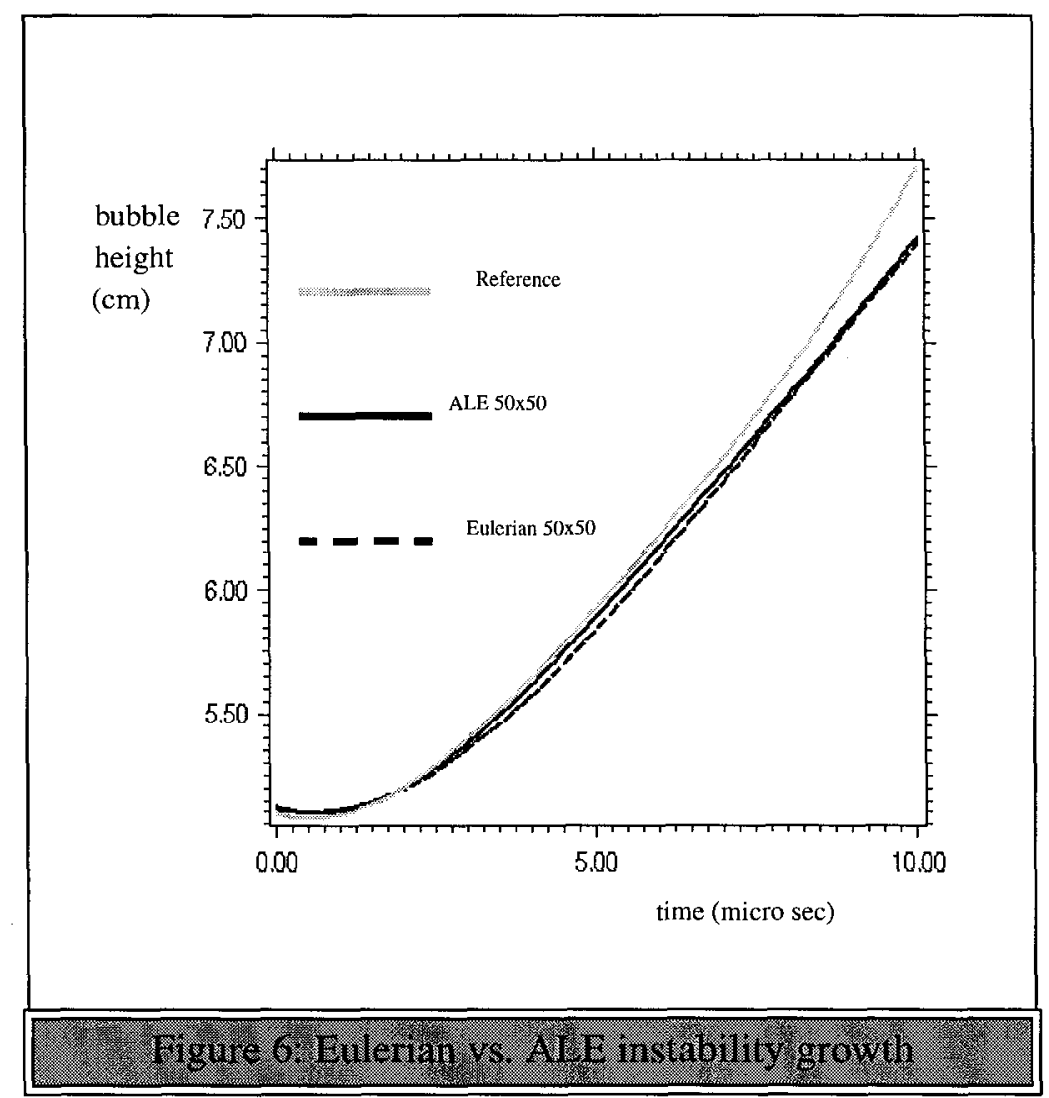

\subsubsection{Energy Balance}

When the mesh moves, some energy is lost due to advection approximations. Both ALE and Eulerian simulations lose energy due to advection, since both use a Lagrangian and an Eulerian step. Over the course of the simulation, internal and potential energy are converted to kinetic energy. The matcrials arc initially in cquilibrium, with the heavier fluid balanced by the light fluid against the acceleration. As instabilities grow, the heavier fluid falls past the light fluid, reducing potential and increasing kinetic energy. ALE simulations captured the conversion of potential energy to kinetic better than Eulerian simulations at low resolution. 
ALE simulations also captured the conversion of internal energy to kinetic better than Eulerian simulations at low resolution. Initially, the problem was balanced so that the pressure gradient was constant throughout the domain, and balanced gravity. This means the internal energy of the less dense material was higher than the internal energy of the more dense material. Both materials became hotter toward the bottom of the domain. The resulting changes in internal energy are better captured by ALE. The increase in kinetic energy was also greater for ALE mesh motion than for Eulerian. Therefore, ALE 
improved the ability of a simulation to capture unresolved kinetic energy.

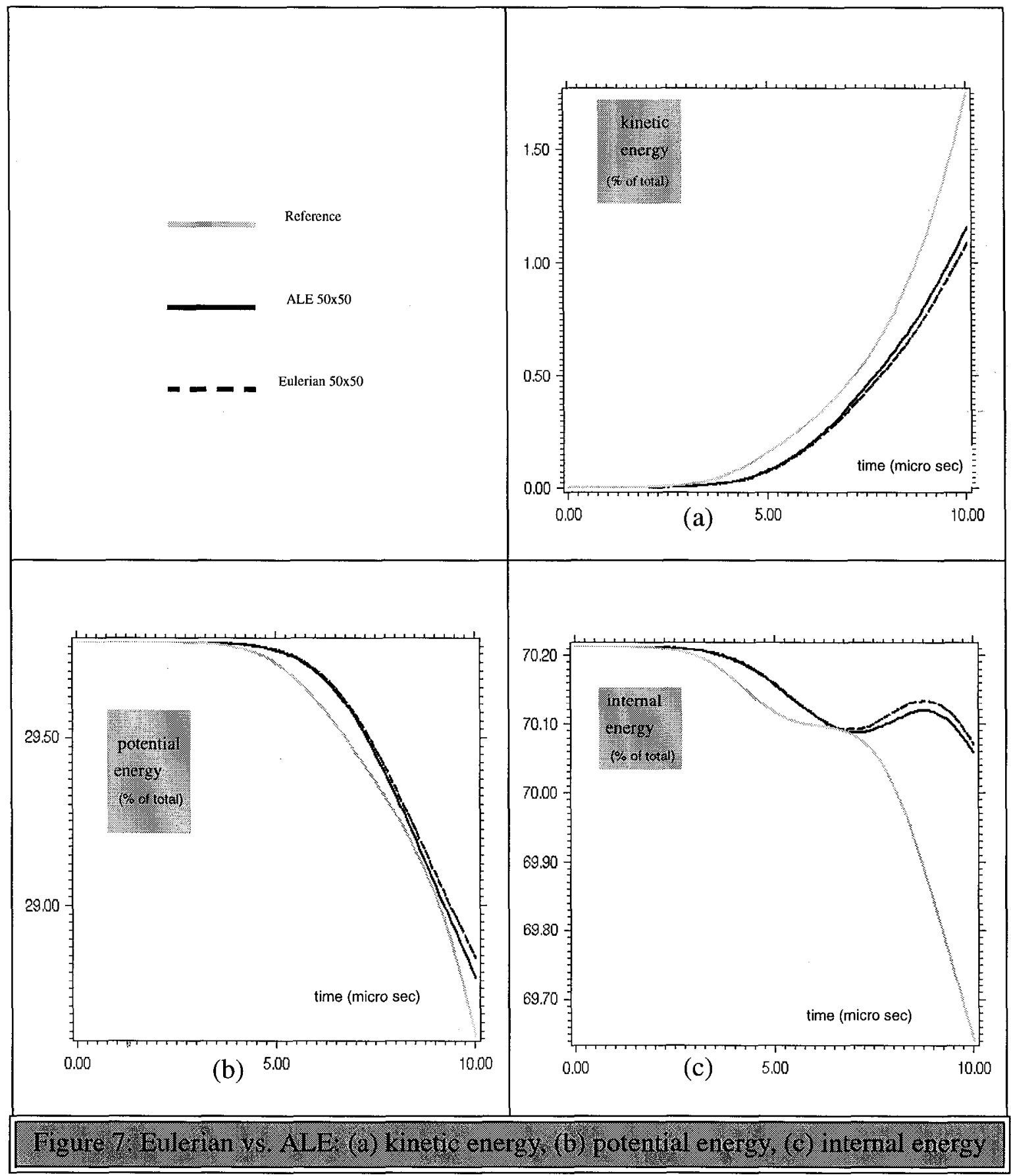

ALE mesh motion captured instability growth and the partitioning of energy into internal, kinetic and potential energy better than Eulerian mesh motion. Many parameters can be changed to alter the way ALE works. The parameters effecting ALE were 
modified one at a time to determine their effect, if any, on the growth of the instability.

For the most part, this effect was minimal.

\subsection{The effect of the Lagrangian step on Rayleigh-Taylor growth}

In the Lagrangian step, the equations of motion are solved on the mesh which moves to follow the fluid. This step can occur one or more times between remap steps, in which the grid is moved past the fluid. Allowing Lagrange subcycling can increase bubblc growth, since the mesh follows the fluid more closely with fewer remaps. In the case

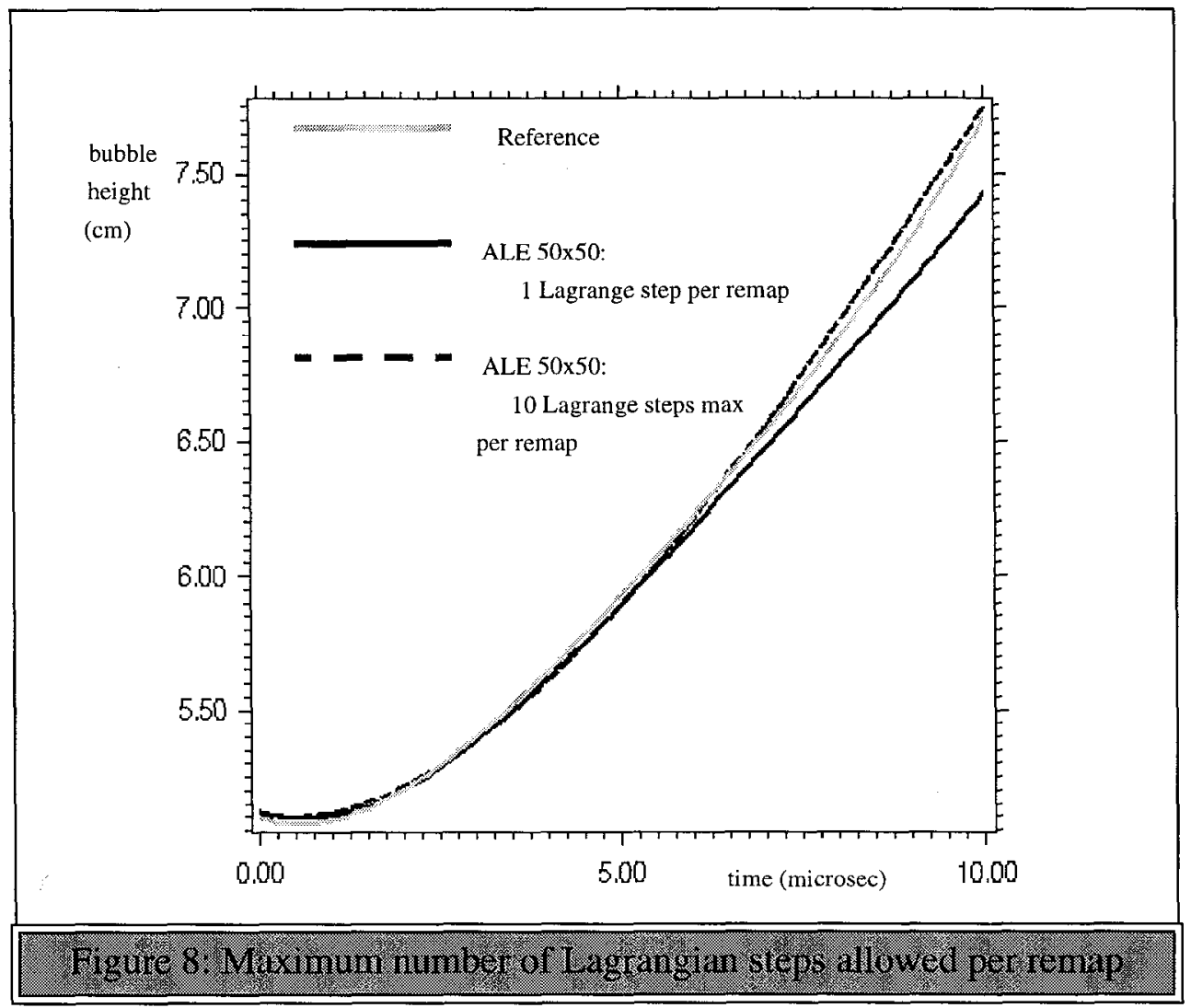

where the maximum number of subcycles was 10 , the actual number of Lagrange steps per remap was only 10 in the early part of the simulation. The mesh followed the fluid in a more Lagrangian fashion until it started to become tangled, and the number of Lagrange steps was reduced. The result was more accurate bubble growth. 


\subsection{The effect of grid relaxation on Rayleigh-Taylor growth}

Solving the equations of motion in a Lagrangian frame of reference allows an ALE mesh to move in response to fluid motion. This improves simulations of complicated flow patterns such as Rayleigh-Taylor instability. However, mesh distortion must be limited since spatial derivatives become inaccurate on a distorted mesh. If the mesh were orthogonal, spatial derivatives would be tied to cartesian spatial derivatives. The derivative of a zone centered quantity would logically be located at the center of the zone face separating two zones. For a non-orthogonal mesh, the spatial derivative of a zone-centered quantity is assumed to be located at a node, and the derivative of a node centered quantity is assumed to be located at a zone center. If a node does not fall in the center of the surrounding zones, inaccuracy is introduced. Therefore, the extent of mesh motion must be limited.

The first step in grid relaxation is to determine which nodes need to move and which do not. Each node is tested individually against two criteria. An angle test compares the smallest angle of the four surrounding a node. The sine of this angle is compared to a threshold angle. In 3 dimensions, a similar test compares the solid angles surrounding a node. 
Reducing the threshold angle $\mathrm{q}$ allowed the instability to grow further, since the zones

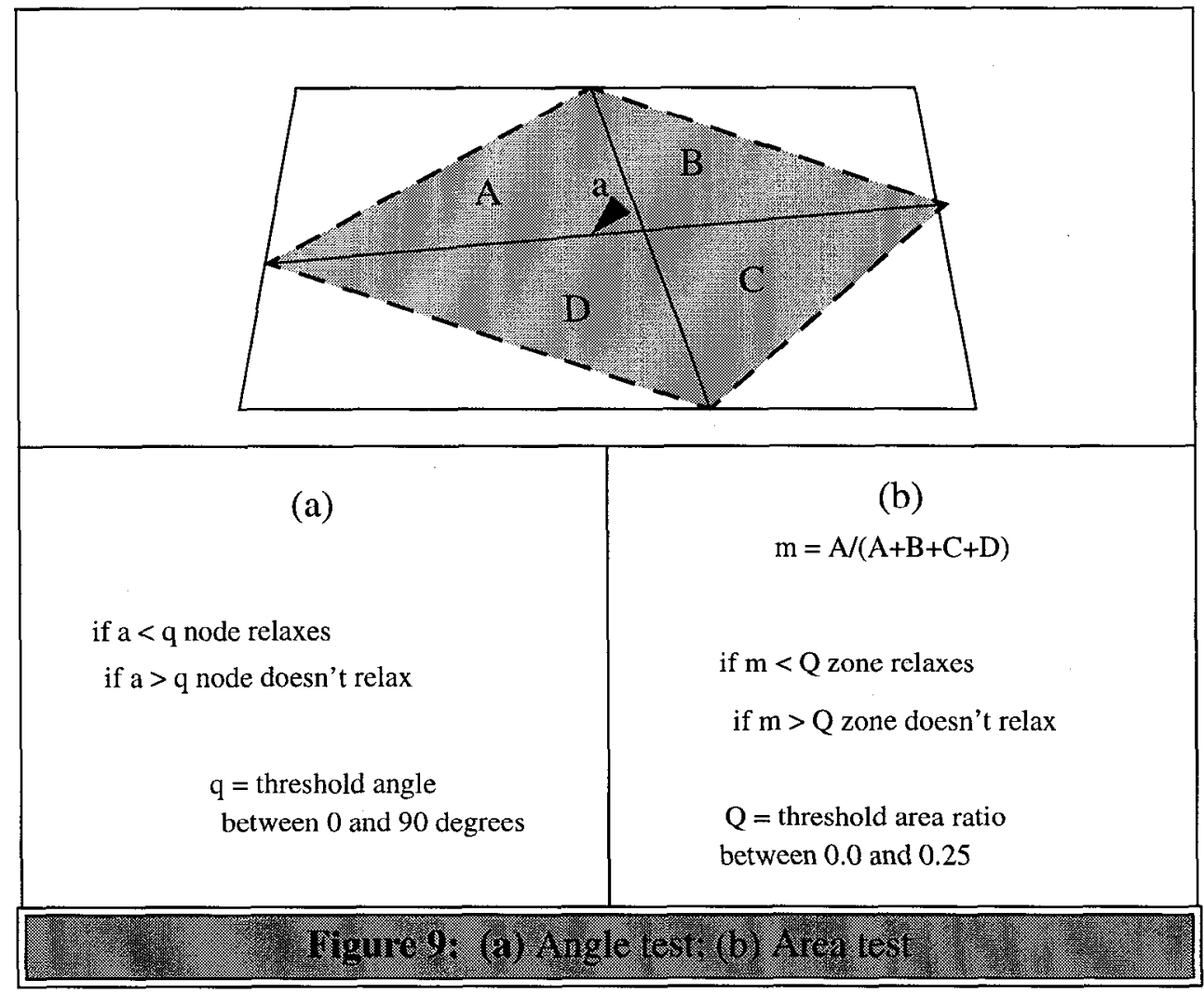


fit the shape of the interface better when small angles were allowed.

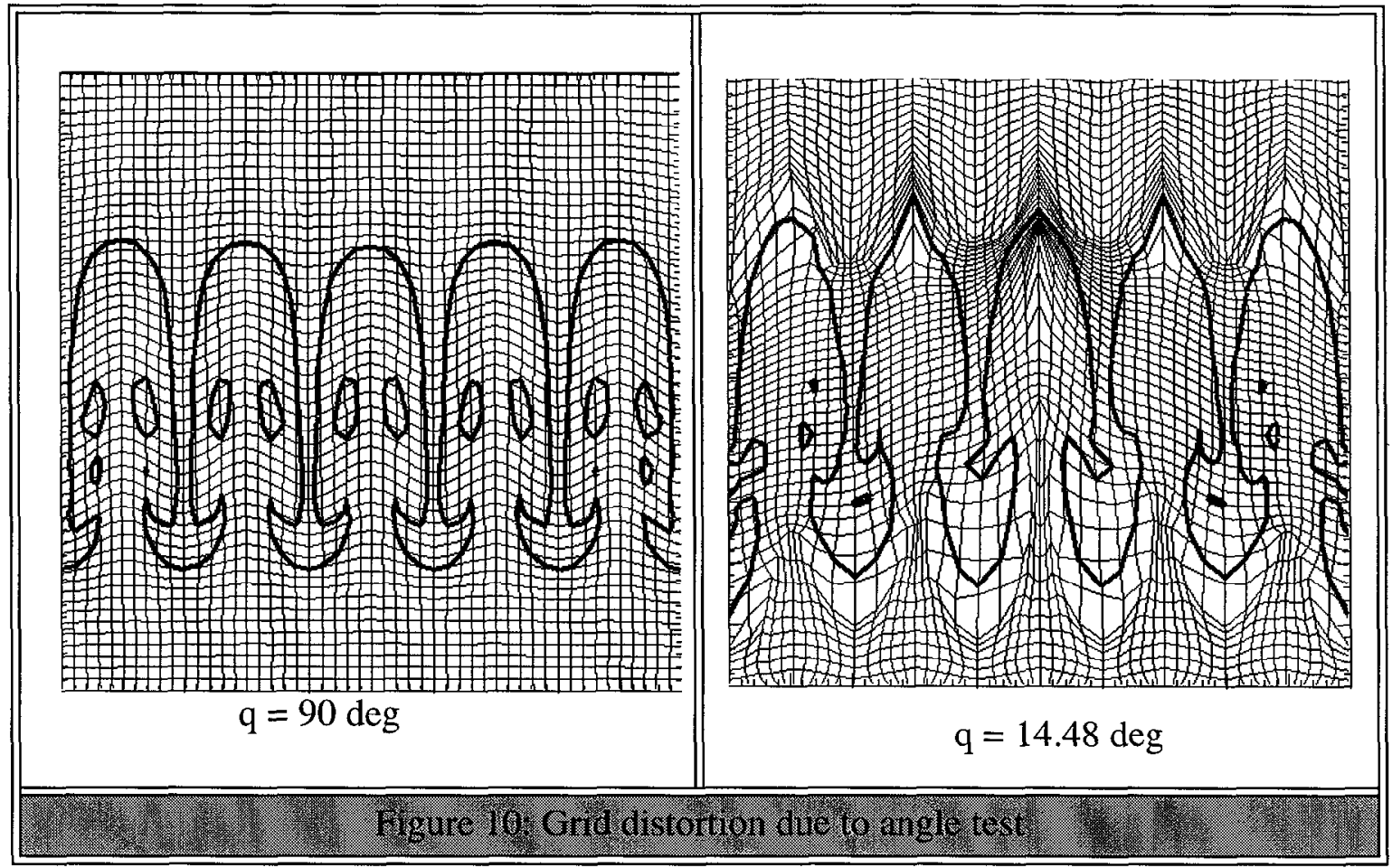

Early in the simulation this made the instability growth more accurate, as can be seen in Fig. 12. But as time went by, the mesh distorted along with the interface, and the instability growth exceeded the more accurate high resolution growth. Reducing the threshold angle made the simulation more Lagrangian, and so the mesh became tangled.

The area test had a similar effect. The area test compares the area of the smallest of the four triangles surrounding a node to the sum of the areas of all four triangles. A similar test in 3 dimensions compares the volumes of the tetrahedra surrounding a node. When the threshold area Q was reduced, an instability could grow faster since the grid distorted further. The area test had far less effect than the angle test however, since it did not allow grid zones to distort to match the shape of the interface as well. 


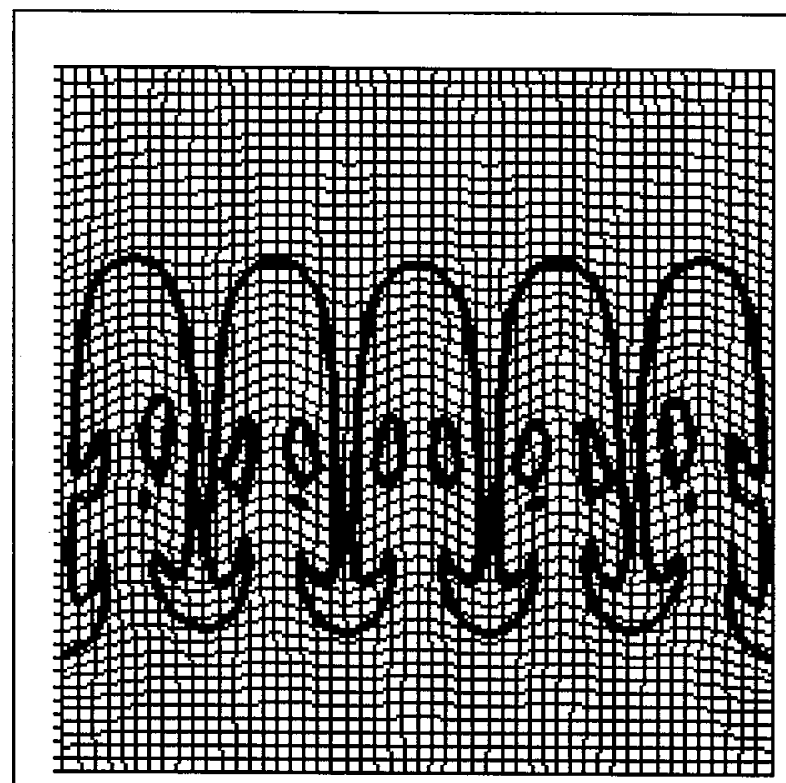

$Q=0.25$

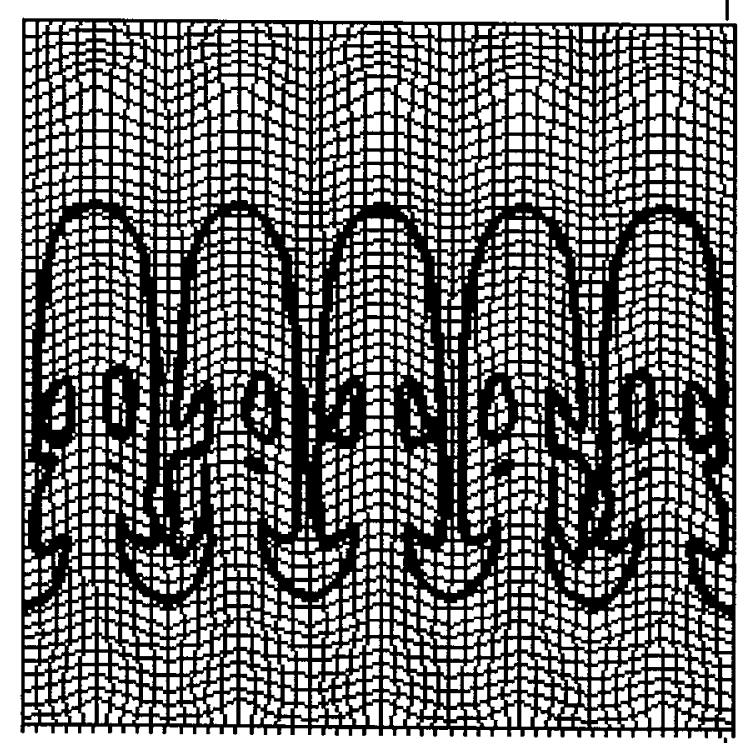

$\mathrm{Q}=0.005$

\section{Figure 11: Grid distoition due to area tesi}

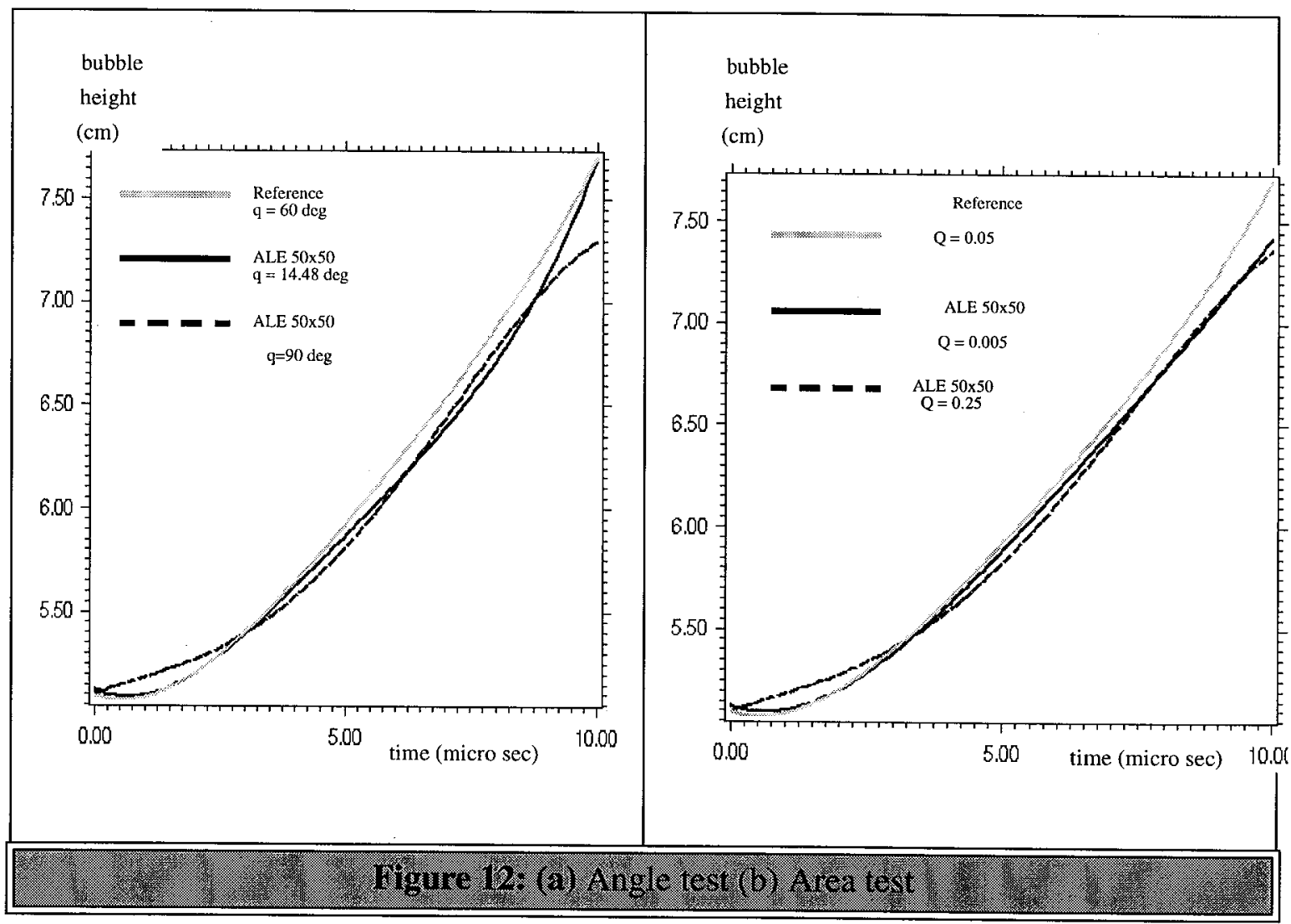


Once the grid nodes are separated into those that will remap and those that will not, the motion of the moving nodes can be effected by several parameters. The solution to the grid relaxation equations (EQ 88 and EQ 89) is taken iteratively. Each iteration brings the mesh closer to the exact solution of the grid relaxation equations. Increasing the number of iterations in the solution caused a slight increase in the instability growth.

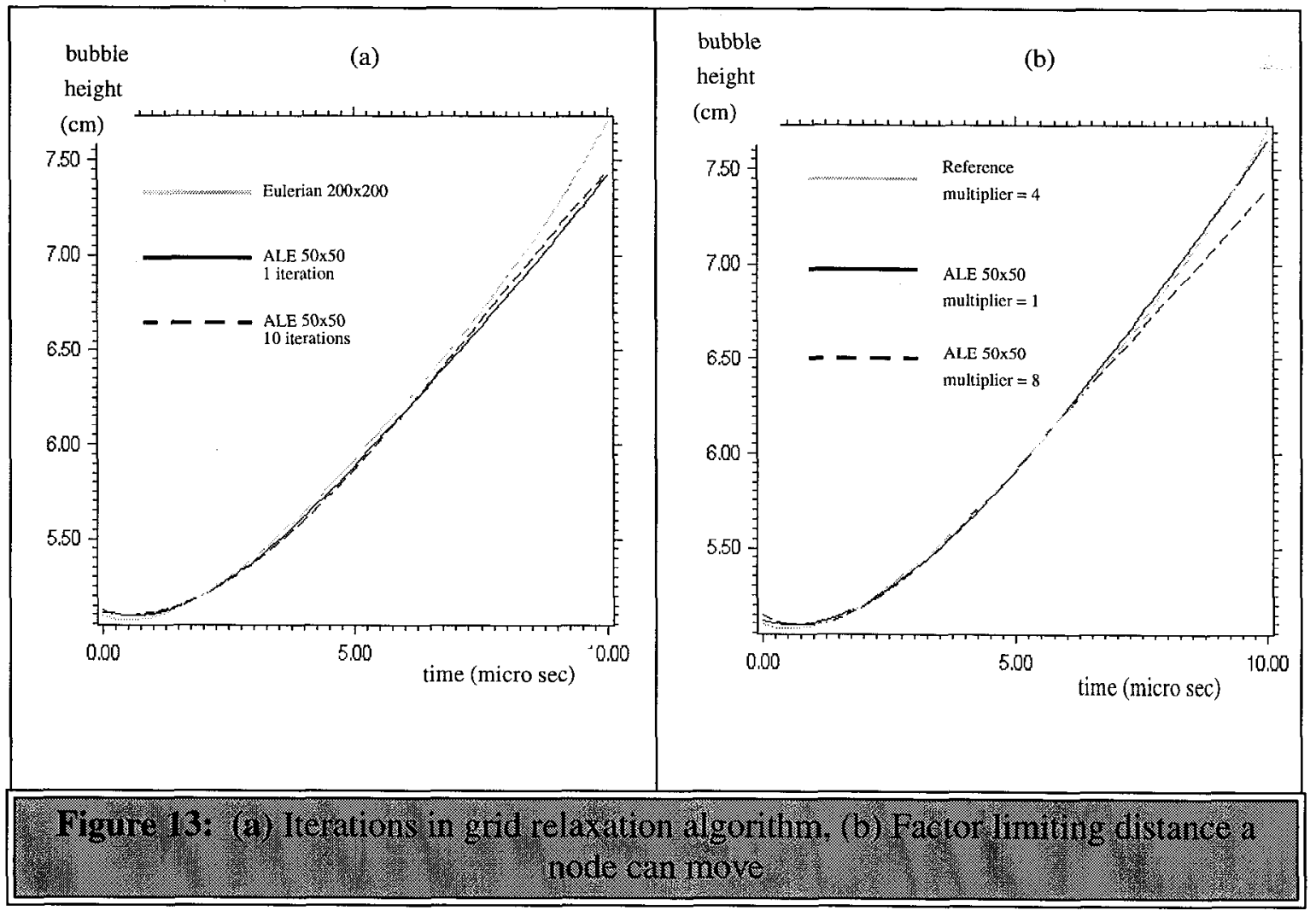

Restrictions can be placed on the distance a grid node can move in one time step by setting a constant factor. During the Lagrangian step, each node moves a distance $l_{1}$ to follow the fluid. The grid relaxation algorithm sets a distance $l_{2}$ that the node should move, perhaps in another direction. The grid relaxation multiplier $r$ is used to limit the distance (but not the direction) a node actually moves. If $l_{2}>r l_{1}$ then $l_{2}=r l_{1}$. The 
parameter $r$ is set for each material region. The typical value of $r$ is 4, but reducing it to 1 increased Rayleigh-Taylor growth. The zones moved a shorter distance when they were remapped, which kept them closer to their positions after the Lagrangian step. Increasing the factor to 8 had less effect.

Regional weights can be used to keep zones in particular parts of the simulation. These weights are applied as factors in the weight functions $W_{i}$ in EQ 88 and EQ 89. In the Rayleigh-Taylor test problem used here, a higher relative weight in one material made nodes stay in that material.

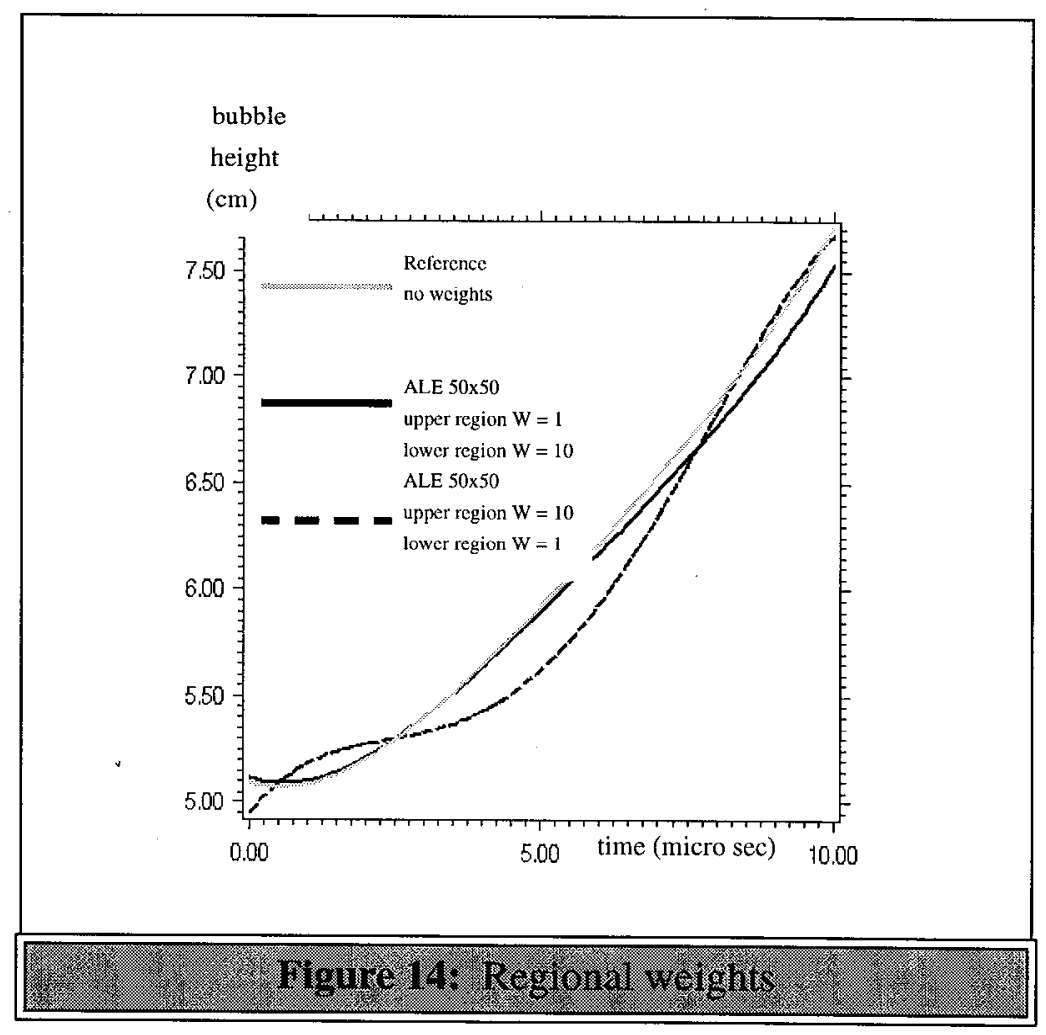

The mesh for the two cases in Fig. 14 can be seen in Fig. 15. More nodes remained 
in the region with the highest weight, but grid distortion occurred, making the simulation less accurate.

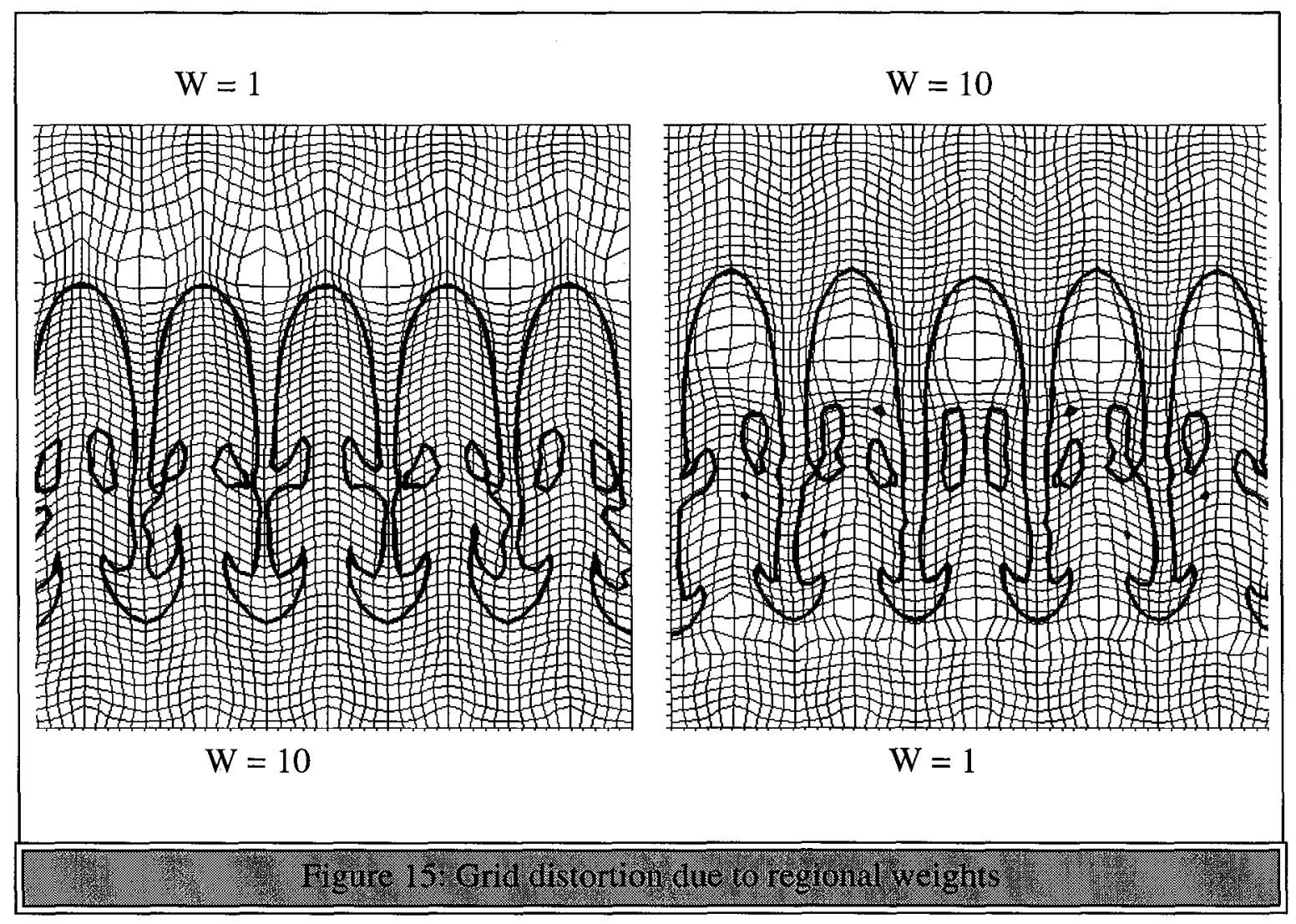

Regional weighting distorted the mesh more than ALE usually allows. This effect can be diminished by averaging the weights of nearest neighbors. Alternatively, the weights of neighboring zones can be replaced with the largest individual weight. 


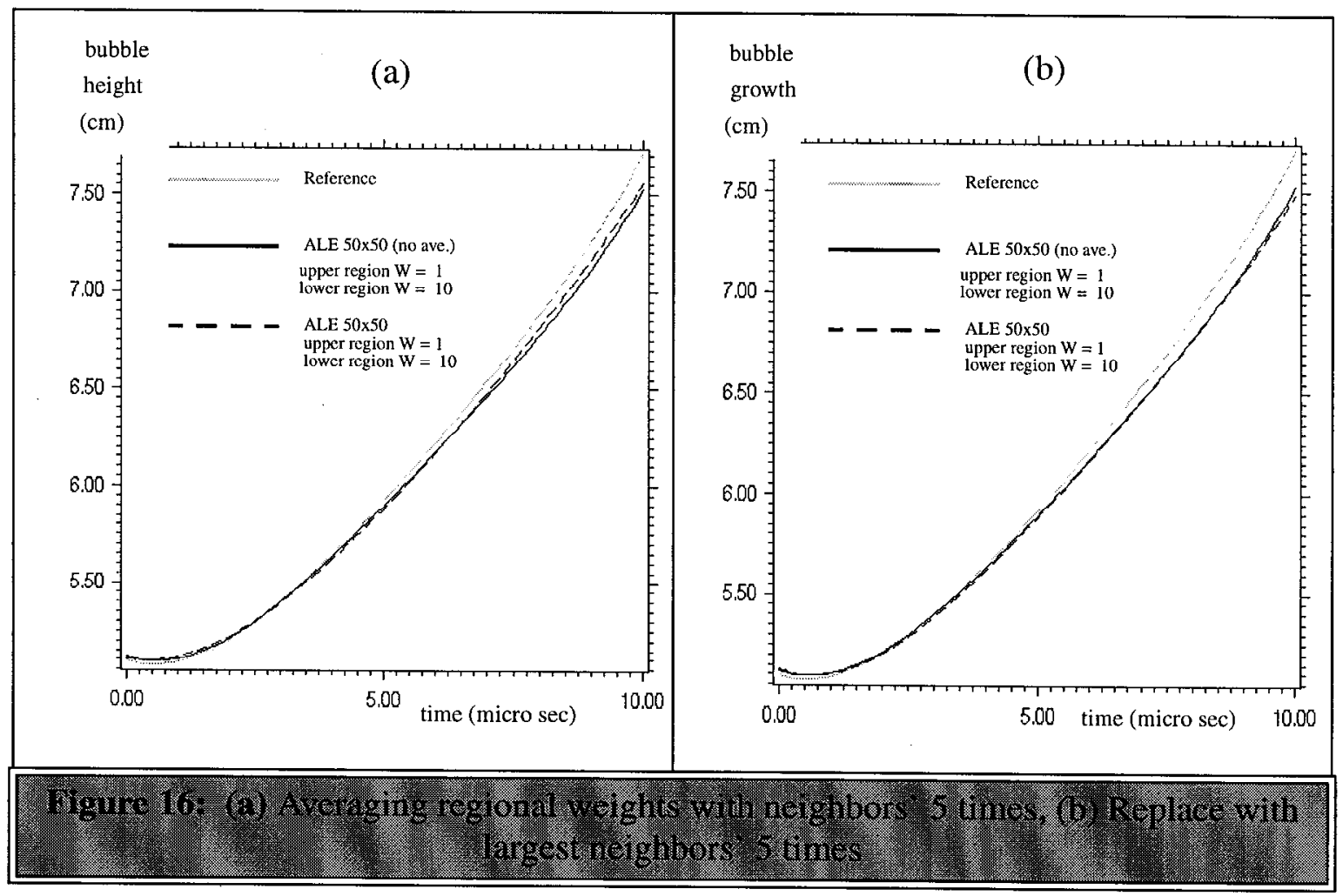

\subsection{The effect of remap on Rayleigh-Taylor growth}

Various advection approximations can be used to move material between zones in response to mesh motion. The Van Leer and Roe Superbee advection schemes are both second order, except when monotonicity constraints force them to be first order. These 
are compared to a strictly first order method. The default advection scheme combined

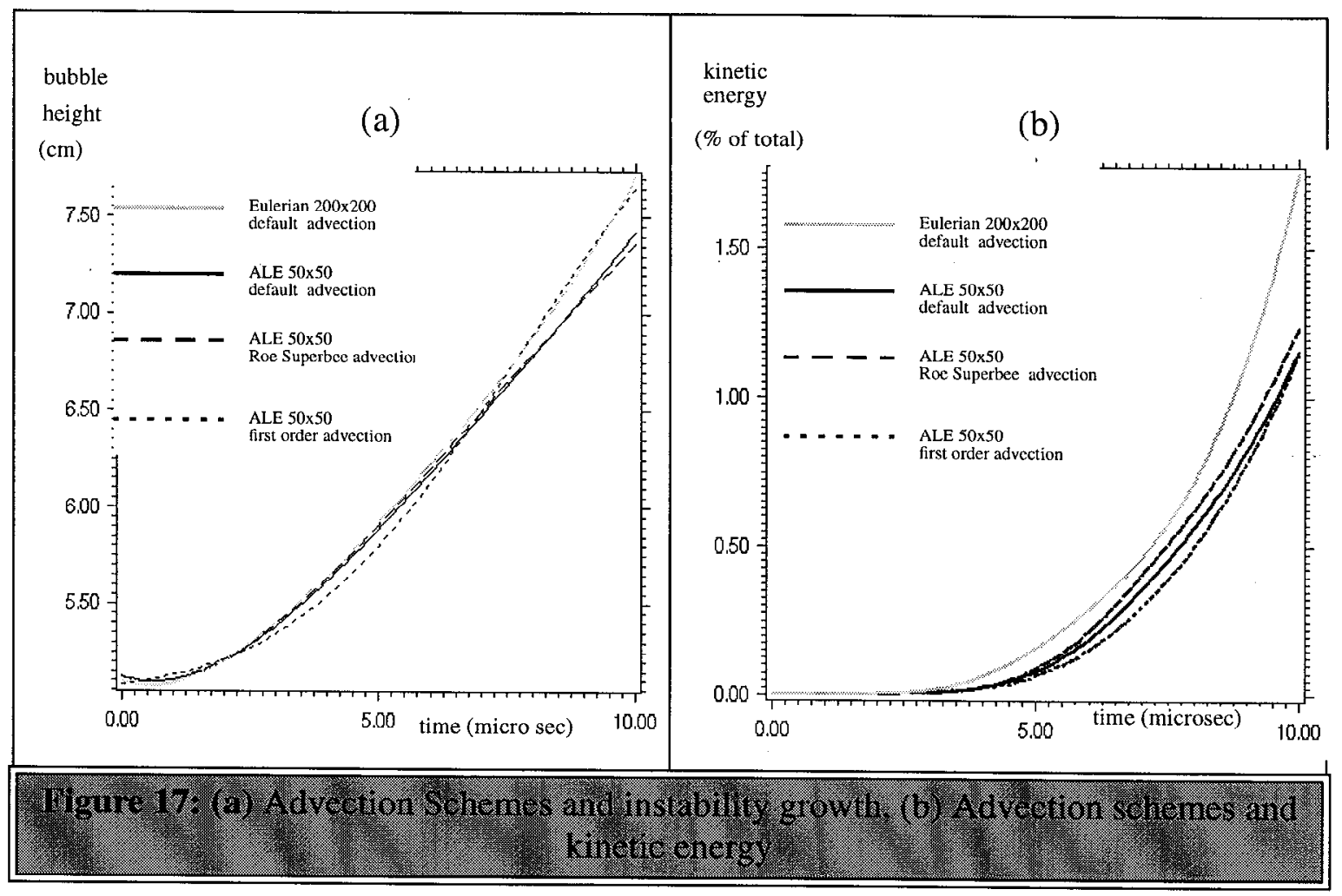

Roe Superbee advection for momentum and Van Leer advection for scalar quantities.

The bubble growth was greater early in the simulation and less later in the simulation for the second order schemes than for the first order scheme. However, the first order scheme converted less internal and potential energy to kinetic energy, making it less accurate in the balance of energy. Roe advection was in gencral more accurate than the default combination.

The order in which advection occurs in the $\mathrm{x}$ and $\mathrm{y}$ directions can also be modified [62]. There are three possible schemes:

1. In one time step advection occurs throughout the domain in the $\mathrm{x}$ direction, then the $\mathrm{y}$ direction. In the next time step, advection occurs in the y direction, then the $\mathrm{x}$ direction. 
2. Advection is performed in the $x$ direction halfway. Then advection occurs in the $y$ direction, after which the advection in the $\mathrm{x}$ direction is finished.

3. Each zone is advected separately. Advection in the $\mathrm{x}$ and $\mathrm{y}$ directions are calculated simultaneously, and corner coupling is lost.

As can be seen in Fig. 18, these schemes made very little difference in total energy loss for the test problem.

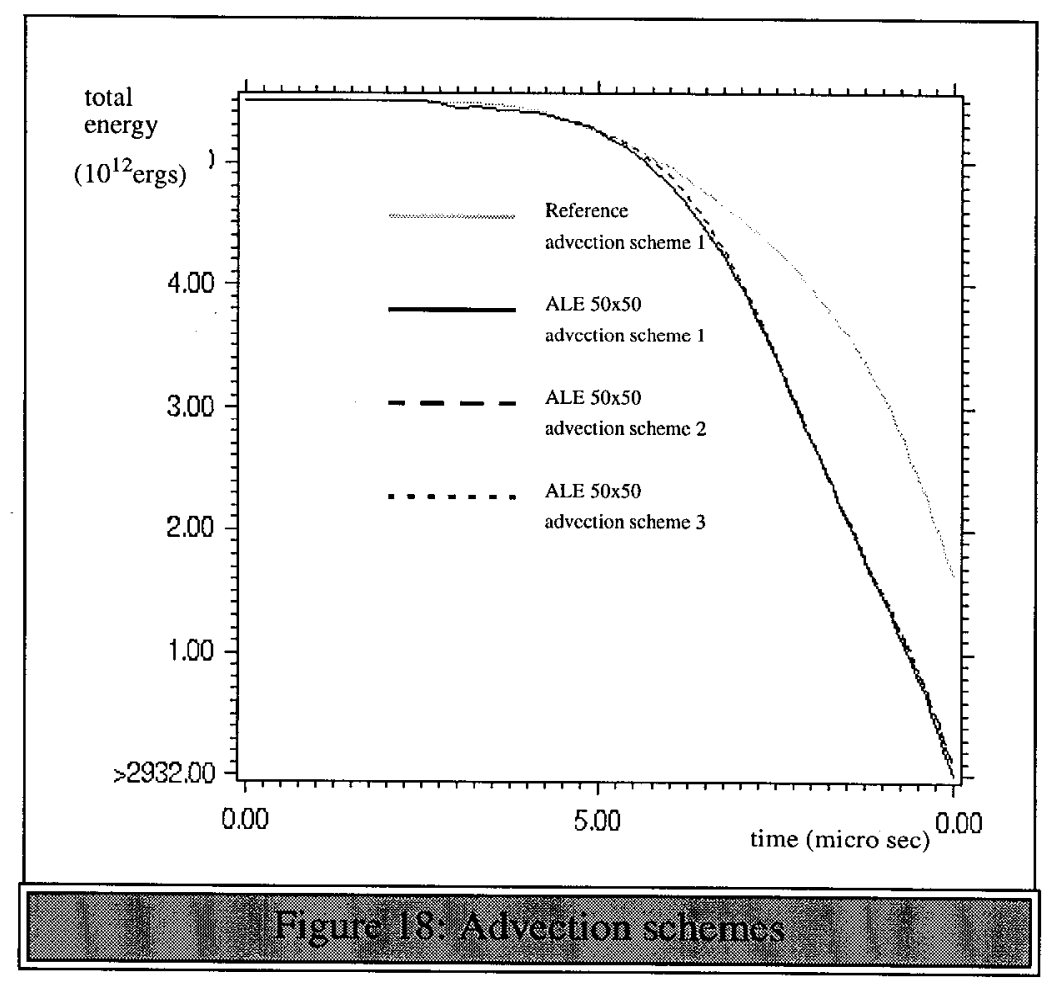

\subsection{Conclusions}

This chapter shows the advantages of the ALE method in Rayleigh-Taylor simulation. Compared to a two step Eulerian method, the ALE technique made a low resolution simulation closer to a higher resolution Eulerian simulation. For the entire domain, the partitioning of kinetic, potential, and internal energy, as well as the bubble growth rate, were all more accurate at lower resolution for the ALE simulation. 
Modifications to ALE were made in the Lagrange step, the grid relaxation step, and the remap step. Several changes in the ALE method caused improvement in the instability simulation relative to the reference case, an Eulerian simulation with 4 times greater resolution. The modifications that had the greatest effect were those that controlled the amount of ALE mesh motion. The mesh could move locally in a more Lagrangian or a more Eulerian fashion, according to local conditions. If the mesh was allowed to follow the fluid in a more Lagrangian fashion, the measurement of instability growth was improved. But where the mesh followed the fluid too closely, mesh distortion became a problem. Therefore, ALE is a compromise between a fixed mesh and one that moves with the fluid.

In the Lagrange step, an increase in the ALE simulation's instability growth occurred when Lagrange subcycling was allowed. The grid relaxation step effected the shape of the instability as it grew. Allowing greater grid distortion by varying the angle test caused faster growth but distorted the interface. Increasing the iterations in the grid relaxation algorithm made relaxation more non-local, and increased instability growth slightly. Restricting the distance a node can move in the relaxation step increased instability growth even more. Regional weights also caused grid distortion, which was minimized by replacing or averaging neighboring weights. The choice of a first order advection scheme increased instability growth, but also increased the loss of kinetic energy due to advection.

The use of ALE mesh motion improved the simulation of Rayleigh-Taylor instabilities. However, ALE and Eulerian simulations were quite close to each other, and the greatest improvement came with increasing resolution. Therefore in cases where high 
resolution cannot be achieved, ALE can make an improvement in Rayleigh-Taylor simulation. 


\section{Large Eddy Simulation and ALE}

The previous chapter examined the usefulness of the Arbitrary Lagrangian-Eulerian (ALE) method in simulations of Rayleigh-Taylor instability with a single initial perturbation mode. This chapter investigates the behavior of a large eddy simulation (LES) in a similar Rayleigh-Taylor simulation with ALE. A multimode initial perturbation is used instead of the simpler single mode initial perturbation, in order to create more unresolved features. LES is intended to describe unresolved kinetic energy in the simulation.

\subsection{Motivation for LES}

When the equations of motion for fluid flow are solved numerically on a grid, it is often impossible to resolve all relevant scales. LES uses a subgrid scale model to approximate the energy in the turbulent eddies which are too small to be resolved by the grid. Without a subgrid scale model, the simulation misses all features of the flow that cannot be resolved by the grid.

In turbulent flow, the fluid moves in circular eddies. Eddies can range in size from the width of the flow to the tiny scale at which the viscosity of the fluid damps fluid motion and converts it to heat. The smallest scale at which a turbulent eddy can exist is known as the Kolmogorov microscale [65]. Due to vortex stretching, large eddies become smaller, until they reach the Kolmogorov microscale. It takes several grid zones to describe a turbulent eddy. The grid spacing is usually far larger than the Kolmogorov microscale, so the conversion of kinetic energy to heat will be missed.

By dimensional analysis, the Kolmogorov microscale $\lambda$ is related to the viscosity $v$ 
and the dissipation rate of kinetic energy $\varepsilon$ by $\lambda \sim\left(\frac{v^{3}}{\varepsilon}\right)^{1 / 4}$. The dissipation rate is related to the largest significant length scale in the flow, $L$, by $\varepsilon-u^{3} / L$ where $u$ is a characteristic velocity of the flow. Therefore,

$$
\frac{\lambda}{L}=\left(\frac{v}{u L}\right)^{3 / 4} \equiv R^{3 / 4}
$$

To resolve the smallest relevant scales in the flow, a simulation needs at least $R^{3 / 4}$ zones per dimension, where $R$ is the Reynolds number of the flow. This means $R^{6 / 4}$ zones are needed for two dimensional simulations and $R^{9 / 4}$ zones are needed for three dimensional simulations. With turbulent flows having Reynolds numbers in the thousands or higher, it is clear that resolving all scales in simulations of turbulent flows is usually too large a problem for today's computers.

In LES, a subgrid scale model approximates the energy in the turbulent eddies which are too small to be resolved by the grid. The turbulence acts like viscosity, dissipating energy from the mean flow. So subgrid scale models often include an eddy viscosity which is added to the molecular viscosity of the fluid. As the mesh spacing becomes smaller and approaches the Kolmogorov microscale, the eddy viscosity disappears, leaving only the molecular viscosity. This means that as the grid spacing goes to zero, the LES becomes a direct numerical simulation (DNS), in which all flow scales are resolved. 


\subsection{History of LES}

Many subgrid scale models have been developed. One of the most well known is the Reynolds Stress model [65]. This defines each component of the velocity $u_{i}$ as the sum of a mean component $\bar{u}_{i}$ and a fluctuating component $u_{i}^{\prime}$.

$$
u_{i}=\bar{u}_{i}+u_{i}^{\prime}
$$

The mean component is a time average defined as

$$
\bar{u}_{i}=\lim _{T \rightarrow \infty} \frac{1}{T} \int_{t}^{(t+T)} u_{i} d t
$$

The entire momentum equation is averaged to find the relationship between the mean and fluctuating velocities. The mean velocity is constant in time, and the average of the fluctuating velocity is zero, so $\overline{\bar{u}}=\bar{u}$ and $\overline{u^{\prime}}=0$. All correlations between mean and fluctuating terms disappear. The fluctuating velocity enters the momentum equation (EQ 13) as the Reynold's stress $\overline{\rho u_{i}^{\prime} u_{j}^{\prime}}$.

$$
\rho \nabla \bullet u=-\nabla p+\nabla \bullet\left(\mu \varepsilon_{i j}-\overline{\rho u_{i}^{\prime} u_{j}^{\prime}}\right)+\rho g
$$

The Reynolds stress represents a new unknown quantity in the equations of motion.

The mixing length model [68] represents the effect of a turbulent eddy moving with respect to the mean flow. The Reynolds stress is approximated from mean quantities.

$$
\overline{\rho u_{x}^{\prime} u_{y}^{\prime}}-\rho l^{2}\left|\frac{\partial}{\partial y} \overline{u_{x}}\right| \frac{\partial}{\partial y} \bar{u}_{x}
$$

The "mixing length" $l$ represents the size of a characteristic turbulent eddy. The eddy 
viscosity is defined as $\mu_{t}=\rho l^{2}\left|\frac{\partial}{\partial y} \bar{u}_{x}\right|$. This viscosity is not a physical attribute of the fluid but a model of the effect of lurbulence on the flow. It represents the effect of small eddies on the mean flow.

Some form of eddy viscosity figures in many subgrid scale models. It describes the dissipative effect of the small scale turbulent eddies on the large scale flow. Eddy viscosity is intended to convert kinetic energy to internal at scales that can be resolved, as shown by the gray line in Fig. 19 . 


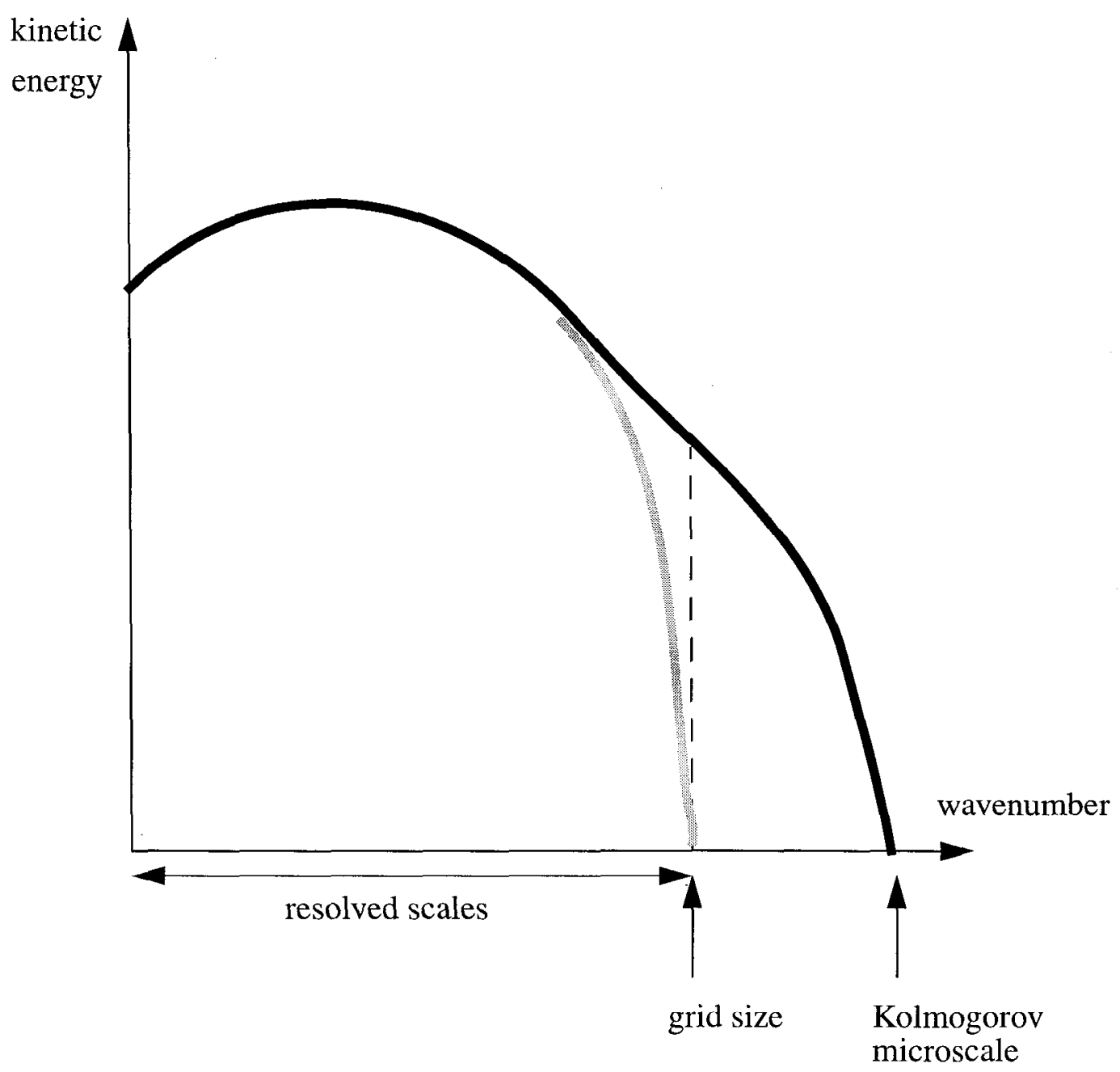

Fig. 19: Eddy viscosity converts kinetic energy to internal at the grid scale, not the unresolved Kolmogorov microscale.

In numerical simulations, some scales can be resolved and some cannot. It makes sense to divide the flow into resolved and unresolved components, rather than mean and fluctuating components. Many subgrid scale models of this type have been developed. A filtering operation can be used to divide the variables into resolved and unresolved components, as the Reynolds time average divides the flow into mean and fluctuating components. 
However, unlike Reynolds averaging, correlations between large and small scale components do not disappear, which leads to additional terms in the equations of motion. Each additional term represents an unknown quantity without an equation associated with it. This is the closure problem of turbulence theory. Closure problems are addressed with further approximations, and the equations of motion are redefined. A set of equations describing the flow down to the grid scale is created to look like the equations of motion for a DNS as much as possible. Another equation of motion describing the subgrid scale kinetic energy is then added.

Various averaging techniques can be used to separate the equations of motion into large scalc and subgrid scale components. The physical parameters of the system (density, velocity, and energy) are defined as the sum of large and subgrid scale components: $f=\langle f\rangle+f^{\prime}$. The large scale components are separated from the subgrid scale components by a convolution product with a filter function $G(x)$.

$$
\langle f(x, t)\rangle=\int_{-\infty}^{\infty} \int_{-\infty-\infty}^{\infty} \int^{\infty} G\left(x-x^{\prime}\right) f\left(x^{\prime}, t\right) d x^{\prime}
$$

the function $G(x)$ should be symmetric, disappear at $\pm \infty$ and normalize to one. The filter is assumed to have the property that $f(\boldsymbol{x})=\bar{f}(\boldsymbol{x})$ whenever $f(\boldsymbol{x})$ is a constant. This assumes that $\int_{-\infty}^{\infty} G(x) d x=1$. Note that if we let $\boldsymbol{x}-\boldsymbol{x}^{\prime}=\boldsymbol{s}$, then

$$
\frac{\partial}{\partial x^{\prime}} G(s, t)=\frac{\partial G \partial s}{\partial s} \frac{\partial x^{\prime}}{\partial s}-\left(\frac{\partial}{\partial s} G(s, t)\right) \text { and } \frac{\partial}{\partial x} G(s, t)=\frac{\partial G \partial s}{\partial s} \frac{\partial}{\partial x}=\frac{\partial}{\partial s} G(s, t) \text { so that }
$$




$$
\frac{\partial G}{\partial x}=-\left(\frac{\partial G}{\partial x^{\prime}}\right)
$$

If $G$ disappears at $\pm \infty$, EQ 16 can be used to show that the filter commutes with spatial derivatives[49].

$$
\begin{aligned}
\overline{\frac{\partial f}{\partial x}} & =\int_{-\infty}^{\infty} G\left(x-x^{\prime}\right)\left(\frac{\partial}{\partial x^{\prime}} f\left(x^{\prime}, t\right)\right) d x^{\prime} \\
& =\left.G\left(x-x^{\prime}\right) f\left(x^{\prime}, t\right)\right|_{-\infty} ^{\infty}-\int_{-\infty}^{\infty}\left(\frac{\partial}{\partial x^{\prime}} G\left(x-x^{\prime}\right)\right) f\left(x^{\prime}, t\right) d x^{\prime} \\
& =\frac{\partial}{\partial x} \int_{-\infty}^{\infty} G\left(x-x^{\prime}\right) f\left(x^{\prime}, t\right) d x^{\prime} \\
& =\frac{\partial}{\partial x} \bar{f}
\end{aligned}
$$

However, this argument breaks down in cases where $G$ is not continuous, as with the step function in EQ 19.

Two common choices for $G(\boldsymbol{x})$ are the Gaussian and the step or top hat function, which use a length scale $\lambda$ as the cutoff length between large and subgrid scales. For example:

$$
\begin{aligned}
& \text { Gaussian: } G(x)=\frac{1}{\pi^{3 / 2} \lambda^{3}} \exp \left(-\left(\frac{x}{\lambda}\right)^{2}\right) \\
& \text { Step function: } G(x)=\left(\begin{array}{c}
1 / \lambda^{3},|x|<\frac{\lambda}{2} \\
0,|x|>\frac{\lambda}{2}
\end{array}\right)
\end{aligned}
$$

The convolution product of the function $f(\boldsymbol{x}, t)$ and the filter function $G(\boldsymbol{x})$ is equal to the inverse transform of the product of their Fourier transforms $\tilde{f}(\boldsymbol{k}, t)$ and $\tilde{G}(\boldsymbol{k})$. Therefore the behavior of the filter function in wave number space indicates which constituent modes of the function survive filtering. In one dimension, taking $\lambda$ to be 1 , the 
two filter functions in EQ 18 and EQ 19 and their transforms are shown in Fig. 20 .

The Gaussian filter has a similar Gaussian transform, which is not uniform in magnitude for all wavenumbers. This filter function emphasizes the smallest wavenumbers in the filtered function. The step function cuts off sharply between resolved and unresolved space, giving all resolved space equal magnitude. But in wavenumber space, fluctuations make some modes positive and others negative. Also, the step filter does not commute with spatial derivatives since it is not continuous. The Gaussian filter does commute with spatial derivatives. 

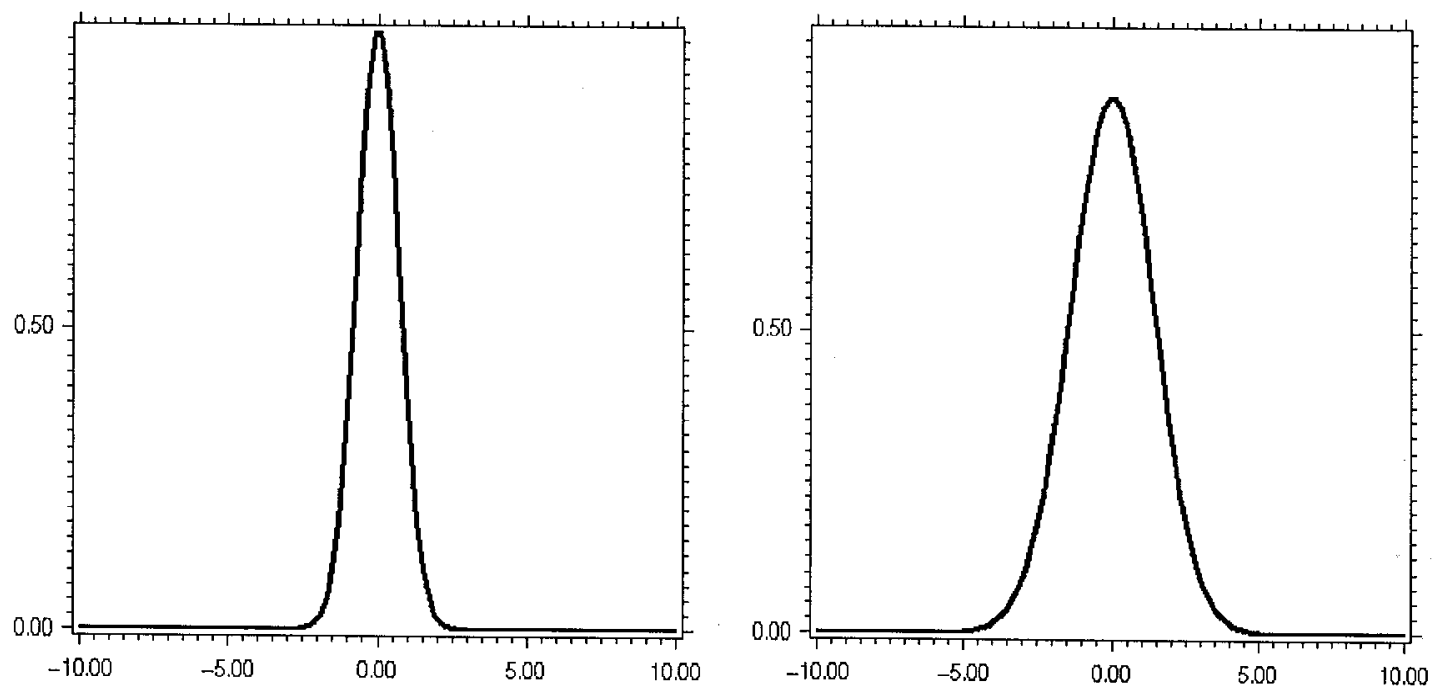

$$
G(x)=\exp \left(-x^{2}\right)
$$

$$
\tilde{G}(k)=\frac{\sqrt{\pi}}{2} \exp \left(-\frac{k^{2}}{4}\right)
$$
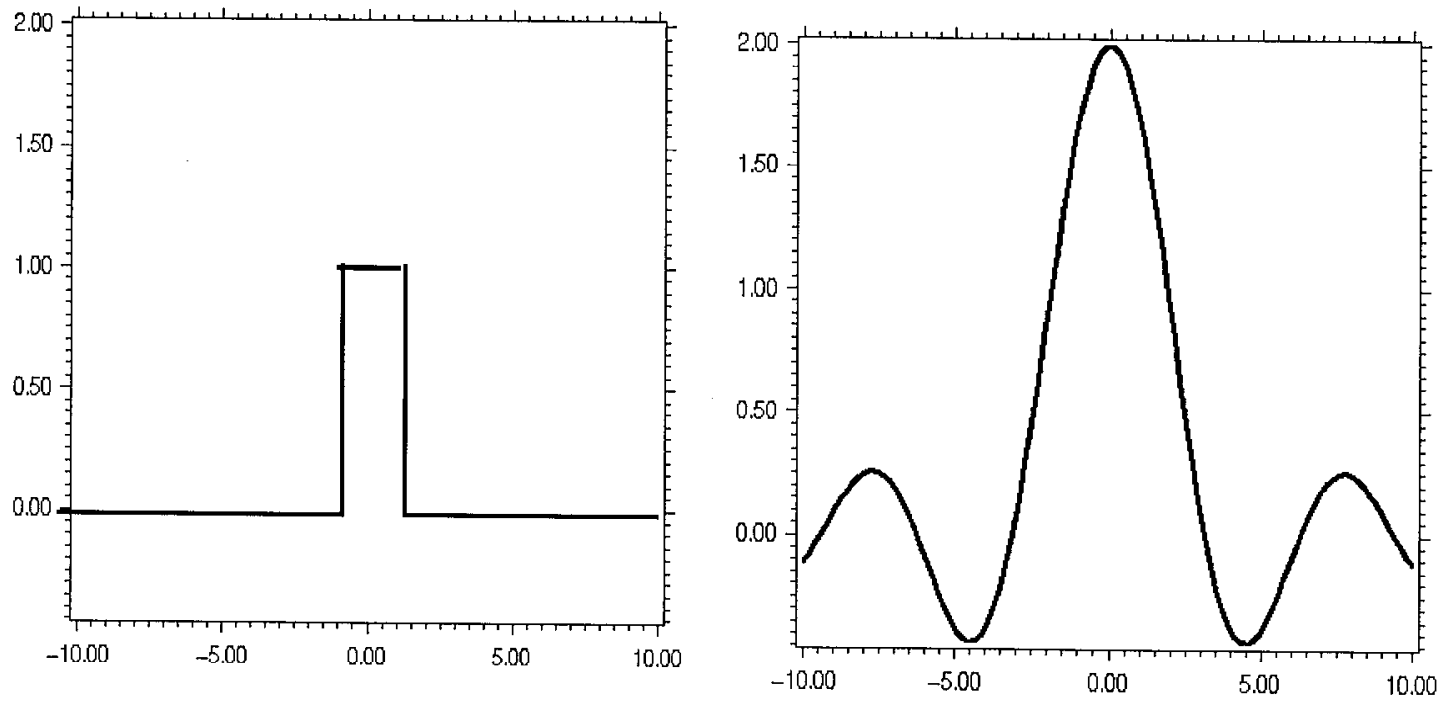

$$
G(x)=\left(\begin{array}{l}
1,|x|<1 \\
0,|x|>1
\end{array}\right)
$$

$$
\tilde{G}(k)=2 \frac{\sin (k)}{k}
$$

Fig. 20: Possible filter functions and their Fourier transforms (with length scale = 1).

An alternative approach to LES is to examine the problem in wave number space rather than physical space. The equations undergo Fourier Transform and the size of the 
component modes which describe the flow can be examined. Again, a filter function can be created to separate the flow into resolved and unresolved components by wavenumber. It is clearer which are large enough to be resolved and which are not. However, the same issues exist in choosing a filter function.

The order of a subgrid scale model is given by the number of new equations of motion which must be solved beyond the original three. A zeroth order model, such as the Reynolds Stress model or the Smagorinsky model [60], has no additional equation of motion but calculates the eddy viscosity from resolved quantities. The Cloutman model [12] uses one additional equation of motion for the subgrid scale kinetic energy $k$. The filtering length scale is proportional to the grid size. This is a first order model. Second order models contain a second variable quantity, such as dissipation of kinetic energy $\varepsilon$ or a turbulent length scale $\lambda$, along with a second additional equation of motion. The filtering length scale is no longer tied to the grid, but to the new variable. The relation between dissipation and length scale is given by $\varepsilon \sim k^{3 / 2} / \lambda$ in the inertial subrange.

This thesis applies LES to simulations that are unusual in several ways. Subgrid scale modeling was created for turbulent flows, but here they are applied to flows that are not yet turbulent but in the unstable stages before turbulence has developed completely. The goal is to use LES as subgrid scale simulation, and improve the accuracy of instability modeling. Also, ALE mesh motion causes the filter definition to vary in space and time. This introduces errors in LES. The importance of those errors is examined in a simple test problem. 


\subsection{Equations of motion}

In the Lagrangian step of the ALE method, the grid moves with the fluid. The equations of motion are solved for one time step. This is done predictor corrector for second order accuracy (see Appendix B Section 5.0 on page 116). The equations in Lagrangian form are:

$$
\text { Conservation of Mass: } \frac{1}{\rho} \frac{d \rho}{d t}=-\nabla \bullet u
$$

$$
\text { Conservation of Momentum: } \rho \frac{d u}{d t}=-\nabla p+\nabla \bullet\left(\mu \varepsilon_{i j}\right)+\rho g
$$

$$
\text { Conservation of Internal Energy: } \rho \frac{d e}{d t}=\nabla \bullet(\kappa \nabla T)-p \nabla \bullet u+\mu \varepsilon_{i j}: \nabla u
$$

\section{Definitions:}

$\frac{d}{d t}=\frac{\partial}{\partial t}+u \cdot \nabla$ Lagrangian derivative

$t$ time

$\rho$ density

$u$ velocity vector

$p$ pressure: in this case, the ideal gas law is used $p=(\gamma-1) \rho e$ where $\gamma=\frac{5}{3}$

$\varepsilon_{i j}=\frac{\partial u_{i}}{\partial x_{j}}+\frac{\partial u_{j}}{\partial x_{i}}-\frac{2}{3} \nabla \bullet u \delta_{i j}$ strain rate tensor

$v=\mu / \rho$ kinematic viscosity of the fluid

$g$ gravitational acceleration

$e$ internal energy per unit mass 
$\kappa$ internal energy diffusivity

$T$ temperature: $T=e / c_{v}$ in this case

$c_{v}=\frac{\partial e}{\partial T}$ heat capacity at constant volume

\subsection{Cloutman model}

The Cloutman model [12] creates a single equation for subgrid scale kinetic energy. This is solved along with the filtered equations of motion at each time step. To find the filtered equations of motion, the unfiltered equations undergo a convolution product with a filter function (see EQ 15). The filter function $G\left(\boldsymbol{x}-\boldsymbol{x}^{\prime}\right)$ is not specified, but a Gaussian is one possibility. The filtering operation is assumed to commute with time and space derivatives. The resulting filtered equations, in Eulerian form, are:

$$
\begin{gathered}
\text { Conservation of Mass: } \frac{\partial}{\partial t}\langle\rho\rangle+\nabla \bullet\langle\rho u\rangle=0 \\
\text { Conservation of Momentum: } \\
\frac{\partial}{\partial t}\langle\rho u\rangle+\nabla \bullet\langle\rho u u\rangle=-\nabla\langle p\rangle+\nabla \bullet\left\langle\mu \varepsilon_{i j}\right\rangle+\langle\rho g\rangle \\
\text { Conservation of Internal Energy: } \\
\frac{\partial}{\partial t}\langle\rho e\rangle+\nabla \bullet\langle\rho u e\rangle=\nabla \bullet\langle\kappa \nabla T\rangle-\langle p \nabla \bullet u\rangle+\left\langle\mu \varepsilon_{i j}: \nabla u\right\rangle
\end{gathered}
$$

Two separate averages are defined for this model. The regular average is written in shorthand form as $\langle f\rangle=\tilde{f}$. A mass weighted average is defined as

$$
\bar{f}=\langle\rho f\rangle /\langle\rho\rangle
$$

so that $\langle\rho f\rangle=\tilde{\rho} \bar{f}$. For all variables except $\rho$ and $p$, filtering is mass weighted so $f=\bar{f}+f^{\prime}$. For $\rho$ and $p$ filtering is not weighted, so $f=\tilde{f}+f^{\prime}$. Because of the 
mass weighting, $\overline{(\bar{f})} \neq \bar{f}$ since $\rho$ is not constant.

A new equation with a new time dependent variable is added to the filtered equations of motion. The new variable is the turbulent kinetic energy per unit mass, $k$. Turbulent kinetic energy is also called subgrid scale kinetic energy, which is a more accurate term in simulations that are not fully turbulent.

To obtain the new equation, the filtered kinetic energy equation is subtracted from the unfiltered kinetic energy equation. The unfiltered kinetic energy equation is found by taking the dot product of the unfiltered velocity with EQ 21 and rearranging terms:

$$
\frac{d}{d t}(\rho K)=-u \bullet \nabla p+u \bullet\left(\nabla \bullet\left(\mu \varepsilon_{i j}\right)\right)+u \bullet \rho g
$$

$K=\frac{1}{2}(u \bullet u)$ is the unfiltered kinetic energy. The filtered kinetic energy equation is obtained by taking the dot product of the filtered velocity with EQ 24, and rearranging similarly:

$$
\frac{d}{d t}(\tilde{\rho} \bar{K})=-\bar{u} \bullet \nabla\langle p\rangle+\bar{u} \bullet\left(\nabla \bullet\left\langle\mu \varepsilon_{i j}\right\rangle\right)+\bar{u} \bullet\langle\rho g\rangle
$$

$K=\frac{1}{2}(\bar{u} \bullet \bar{u})$ is the filtered kinetic energy.

Many approximations are made to simplify the filtered equations of motion and the subgrid scale kinetic energy equation. Closure approximations are adopted to treat unknown correlation terms. This particular subgrid scale model is intended to be as simple as possible, to minimize computational effort.

The left side of the filtered momentum and energy equations contain triple products with $\rho$ and $u$. These can be expanded to: 


$$
\langle\rho u f\rangle=\tilde{\rho}\left(\overline{\bar{u} \bar{f}}+\overline{u^{\prime} \bar{f}}+\overline{\bar{u} f^{\prime}}+\overline{u^{\prime} f^{\prime}}\right)
$$

Zero is added to the right hand side of this equation in the form of $\tilde{\rho} \bar{u} \bar{f}-\tilde{\rho} \bar{u} \bar{f}$, and the terms are redistributed.

$$
\langle\rho u f\rangle=\tilde{\rho} \bar{u} \bar{f}+\tilde{\rho}\left((\overline{\bar{u} \bar{f}}-\bar{u} \bar{f})+\overline{u^{\prime} \bar{f}}+\overline{\bar{u} f^{\prime}}\right)+\tilde{\rho} \overline{u^{\prime} f^{\prime}}
$$

The Leonard terms $(\overline{\bar{u}} \bar{f}-\bar{u} \bar{f})$ and the cross terms $\left(\overline{u^{\prime} \bar{f}}+\overline{\bar{u} f^{\prime}}\right)$ are neglected in this model. For some choices of filter, the Leonard terms are equal to zero. If the distribution of the fluctuations $f^{\prime}$ is Gaussian, then $\bar{f}^{\prime}=0$ and therefore $\overline{(\bar{f})}=\bar{f}$, so the cross tcrms also vanish. In cases where the Leonard and cross terms do not disappear, several closure approximations have been used for them [7][42][49]. These are not employed here, for the sake of simplicity.

The flux gradient approximation is used for subgrid scale correlation terms. This assumes the transport length scale must be small compared to the length over which the gradient of the transported quantity is significant [14].

$$
\overline{f^{\prime} u^{\prime}}=-D_{t} \nabla \bar{f}
$$

The turbulent diffusivity $D_{t}$ depends on what $f$ is: for velocity, $D_{t}$ is viscosity $\mu_{t}$. For internal energy, $D_{t}$ is thermal diffusivity given by $\frac{\mu_{t}}{P r_{t}}$. The turbulent Prandtl number $P r_{t}$ is taken to be 0.7 . This value is the Prandtl number for an ideal gas, so the subgrid scale turbulent eddies are assumed to be homogeneous and isotropic.

The flux gradient approximation is used in the viscous heating term in the model for the Reynolds stress tensor. 


$$
\tilde{\rho} \overline{u_{i}^{\prime} u^{\prime}}{ }_{j}=-p_{i} \delta_{i j}+\mu_{t} \overline{\varepsilon_{i j}}
$$

The turbulent pressure is given by $p_{t}=\frac{2}{3} \tilde{\rho} k$, assuming the subgrid scale turbulence behaves like an ideal gas, where pressure is $p=\frac{2}{3} \rho e$. The turbulent viscosity is given by dimensional analysis as $\mu_{t}=A \rho \sqrt{k} \lambda$, where $\mathrm{A}$ is an arbitrary constant and $\lambda$ is the grid scale.

Sink and source terms are formulated to describe the creation and destruction of turbulent kinetic energy. They appear with opposite signs in the turbulent kinetic energy and internal energy equations, to preserve energy conservation.

A term for the dissipation of turbulent kinetic energy comes from the Kolmogorov energy spectrum [65]. This assumes $E(\zeta) \sim \varepsilon^{2 / 3} \zeta^{-5 / 3}$ in the inertial subrange above the Kolmogorov microscale. $\varepsilon$ is the dissipation rate of turbulent kinetic energy, $\zeta=1 / \lambda$ is the wavenumber, and $E$ is kinetic energy density such that $k=\int_{\zeta}^{\infty} E(\zeta) d \zeta \sim \varepsilon^{2 / 3} \lambda^{2 / 3}$. Solving for the turbulent dissipation rate, $\varepsilon \sim \frac{k^{3 / 2}}{\lambda}$ [2]. The term for the dissipation of turbulent kinetic energy is $D \tilde{\rho} \frac{k^{3 / 2}}{\lambda}$, where D is an arbitrary constant.

A term to represent the creation of turbulent kinetic energy due to buoyancy, $B \mu_{t}\left(\frac{\nabla \tilde{\rho}}{\tilde{\rho}} \cdot \frac{\nabla \tilde{p}}{\tilde{\rho}}\right)$, was developed by Cloutman [12]. Since buoyancy causes instability only when $\nabla \rho \cdot \nabla p<0$, this term is set to zero if it is positive. B is an arbitrary constant. 
Molecular viscosity is neglected because it is assumed to be much smaller than turbulent viscosity. This implies the grid scale will always be much larger than the Kolmogorov scale, at which molecular viscosity becomes significant. Also, terms involving gravitational fluctuations in the turbulent kinetic energy equation are neglected.

This set of approximations is rather extreme, and many more complicated LES techniques have been developed for other studies. But the Cloutman model was judged to be useful in examining the effect of ALE mesh motion on subgrid scale energy, and its consequences to Rayleigh-Taylor instability simulation.

\subsection{New equations of motion}

The new equations of motion, back in Lagrangian form, are:

$$
\begin{gathered}
\text { Conservation of Mass: } \frac{d}{d t} \tilde{\rho}=-\tilde{\rho} \nabla \bullet \bar{u} \\
\text { Conservation of Momentum: } \tilde{\rho} \frac{d}{d t} \bar{u}=-\nabla\left(\tilde{p}+p_{t}\right)+\nabla \bullet\left(\left(\mu+\mu_{t}\right) \bar{\varepsilon}_{i j}\right)+\tilde{\rho} \bar{g} \\
\text { Conservation of Internal Energy: } \\
\tilde{\rho} \frac{d}{d t} \bar{e}=\nabla \bullet(\kappa \nabla \bar{T})+\nabla \bullet\left(\kappa_{t} \nabla \bar{e}\right)-\tilde{p} \nabla \bullet \bar{u}+\mu \bar{\varepsilon}_{i j}: \nabla \bar{u}+S I N K-S O U R C E \\
\text { Conservation of Turbulent Kinetic Energy: } \\
\tilde{\rho} \frac{d k}{d t}=\nabla \bullet\left(\left(\mu+\mu_{t}\right) \nabla k\right)-p_{t} \nabla \bullet \bar{u}+\mu_{t} \bar{\varepsilon} i j: \nabla \bar{u}-S I N K+S O U R C E \\
S I N K=D \tilde{\rho} \frac{k^{2}}{\lambda} \\
S O U R C E=-B \mu_{t}\left(\frac{\nabla \tilde{\rho}}{\tilde{\rho}} \cdot \frac{\nabla \tilde{p}}{\tilde{\rho}}\right)_{i f<0} \\
\text { Turbulent Pressure: } p_{t}=\frac{2}{3} \tilde{\rho} k
\end{gathered}
$$


Turbulent Viscosity: $\mu_{t}=A \rho \sqrt{k} \lambda$

(EQ 40)

Turbulent Diffusivity: $\kappa_{t}=\frac{\mu_{t}}{P r_{t}}$

\subsection{Stochastic backscatter}

Subgrid scale turbulent fluctuations add a random component to large scale motion.

LES without a model for stochastic backscatter misses this component. A large scale flow which is initially symmetric remains symmetric throughout the simulation. This is not typical of real flows, so stochastic backscatter can be added to the model to simulate the effect of unresolved fluctuations on resolved scales. There are many ways to do this. Leith [40] added a random acceleration term to the momentum equation. Schumann [58] added a random component to temperature and velocity fields.

The current study adds a random perturbation to the velocity field in calculation of the strain rate tensor only. Galilean invariance is preserved. In the strain rate tensor $\bar{\varepsilon}_{i j}=\frac{\partial \bar{u}_{i}}{\partial x_{j}}+\frac{\partial \bar{u}_{i}}{\partial x_{i}}-\frac{2}{3} \frac{\partial \bar{u}_{k}}{\partial x_{k}} \delta_{i j}$, which appears in the conservation equations for momentum (EQ 34), internal energy (EQ 35), and turbulent kinetic energy (EQ 36), a random velocity $u_{i}^{R}$ is added to the filtered velocity $\overrightarrow{u_{i}}=\vec{u}_{i}+u_{i}^{R}$.

The magnitude of $u_{i}^{R}$ is related to the subgrid scale kinetic energy $k$. 


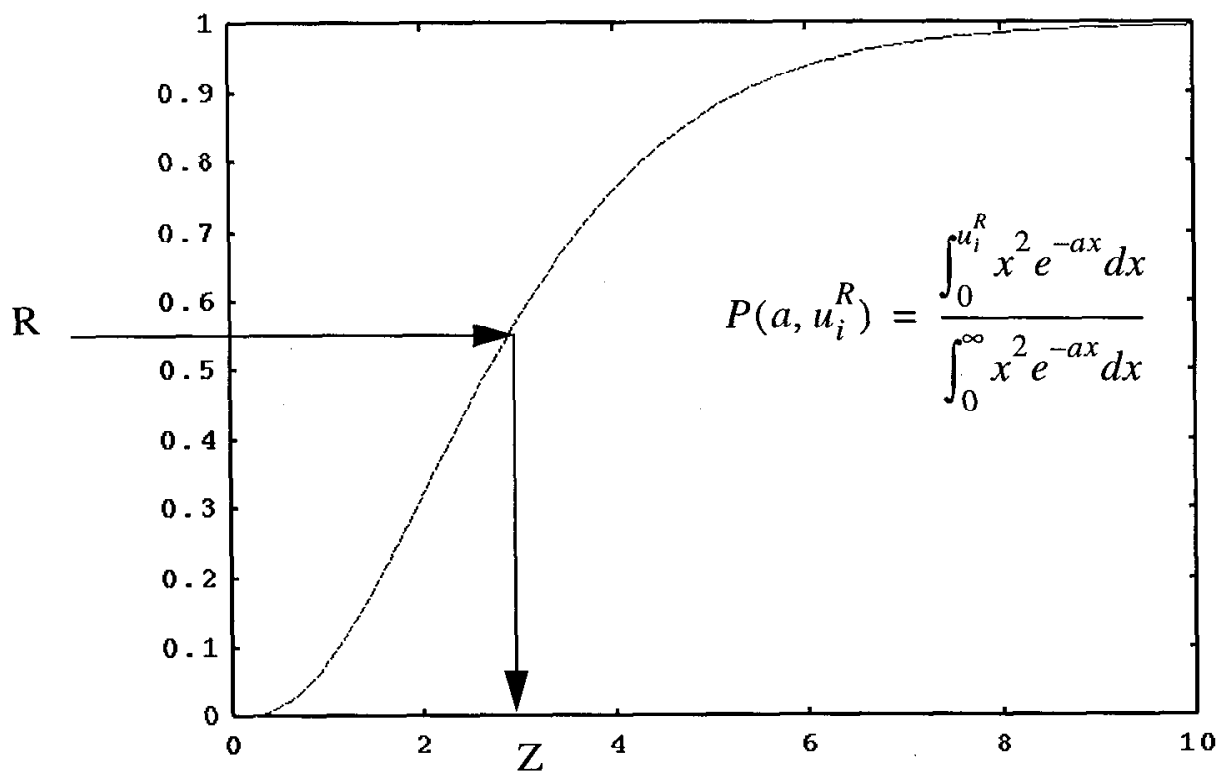

The cumulative distribution function of the random component of the velocity field is chosen to be $P\left(a, u_{i}^{R}\right)$ since it can be solved by integration by parts.

$$
P\left(a, u^{\prime}\right)=1-e^{-a u^{\prime}}\left(1+a u^{\prime}+\frac{1}{2} a^{2} u^{\prime 2}\right)
$$

The mean value is $\bar{x}=\frac{3}{a}$, so we chose $\bar{x}=\alpha_{B S} \sqrt{k}$ to determinc $a=\frac{\alpha_{B S}}{3} \sqrt{k}$. The constant $\alpha_{B S}$ is arbitrary. To find the random velocity fluctuation, a random number $R$ between 0 and 1 is found [53] which replaces $P(z)$. Solving for $u_{i}^{R}$,

$$
-a u_{i}^{R}+\ln \left(1+a u_{i}^{R}+\frac{1}{2}\left(a u_{i}^{R}\right)^{2}\right)=\ln (1-R)
$$

The natural log on the left is expanded in a Taylor Series to third order. Terms above cubic were discarded. The resulting velocity fluctuation term is 


$$
u_{i}^{R}=\frac{\alpha_{B S} \sqrt{k}}{3}(-6 \ln (1-R))^{1 / 3}
$$

Random angles are chosen to give the direction of the velocity fluctuation over a unit sphere. In 2 dimensions, one random angle is required, and in 3 dimensions, two are required.

\subsection{Rayleigh-Taylor instability test problem}

The choice of initial perturbations for a test problem in Rayleigh-Taylor instability is wide. A single mode sinusoidal perturbation can be well cnough resolved to make LES unnecessary. This type of simulation was used in the previous chapter to examine the effects of ALE on Rayleigh-Taylor simulation. A purely random interface could have component modes that range in wavelength from larger than the size of the domain to much smaller than a zone. The effect of unresolved modes on the resolved modes can be studied this way. As resolution is increased, more modes will be resolved but there will be even smaller modes still not resolved. A third approach is to choose a limited number of resolved modes in the initial perturbation. The coupling of these modes may create unresolved modes, but increasing resolution should eventually resolve everything. This type of simulation was used in this chapter, to study the use of LES with ALE in simulating the unresolved effect of mode coupling on resolved modes.

The problem chosen for this study contained 4 modes. They were all resolved by at least 10 zones per wavelength at the lowest resolution studied (50x50). The ideal gas

equation of state was used $\left(p=(\gamma-1) \rho e\right.$ with $\left.\gamma=\frac{5}{3}\right)$. The density ratio was 2.0 to 1.0 in a uniform downward gravitational field of magnitude 1.0. The domain was 10.0 
by 10.0, and all four boundaries were walls with slip conditions, so fluid motion was allowed parallel to the boundaries but not perpendicular to them. The fluid interface had the following shape:

$$
y=5.0+\sum_{i=1,4} A_{i} \cos \left(\frac{2 \pi x}{L_{i}}+\varphi_{i}\right)
$$

The values of the amplitude, wavelength and phase are given in Table 2. The amplitudes were random numbers between 0.0 and 0.1 .

Table 2: component modes in initial material interface

\begin{tabular}{|l|c|l|l|}
\hline \multicolumn{1}{|c|}{$\mathrm{i}$} & \multicolumn{1}{c|}{$A_{i}$} & \multicolumn{1}{c|}{$L_{i}$} & \multicolumn{1}{c|}{$\varphi_{i}$} \\
\hline \hline 1 & $2.533581893 \mathrm{e}-02$ & 2.0 & $\pi / 2$ \\
\hline 2 & $9.346853101 \mathrm{e}-03$ & 3.0 & 0 \\
\hline 3 & $6.084968907 \mathrm{e}-02$ & 4.0 & $\pi / 2$ \\
\hline 4 & $9.034202601 \mathrm{e}-02$ & 5.0 & 0 \\
\hline
\end{tabular}

From time 1.0 to 10.0 , the shape of the interface evolved as the bubbles and spikes grew. The resolved modes coupled to create smaller modes, which were better resolved at higher resolutions. The effect of these smaller features was to slow the growth of the larger modes. 
time $=10.0$

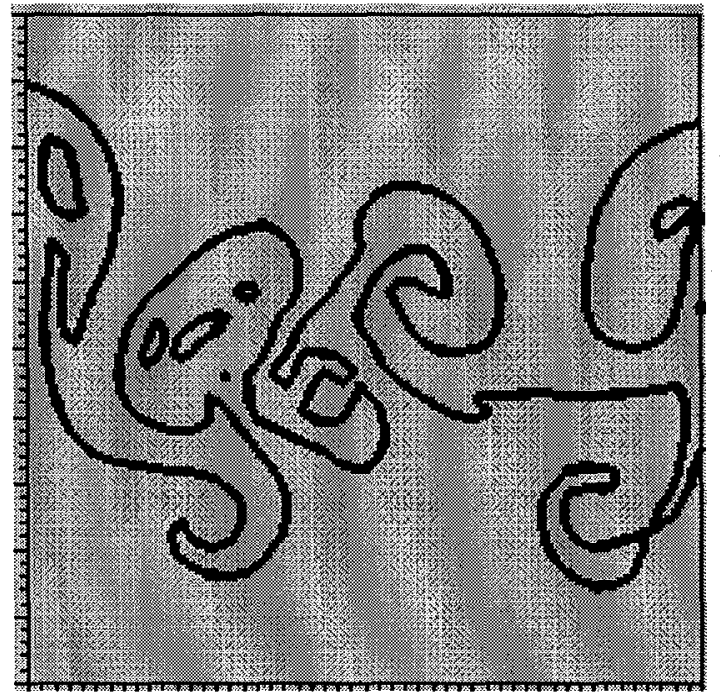

$50 \times 50$

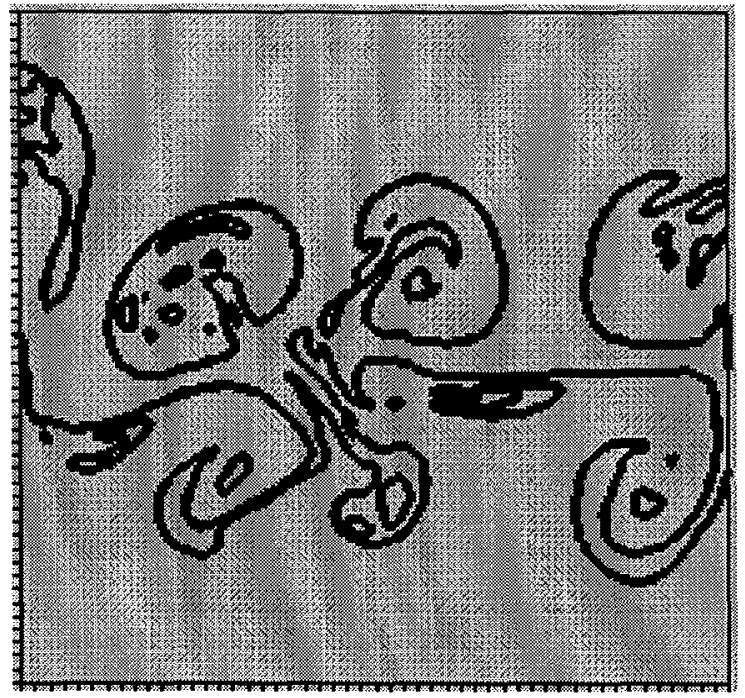

$100 \times 100$

Fig. 21: At higher resolution more small scale features can occur

A three dimensional simulation was created with interface perturbations in both the $\mathrm{x}$ and $\mathrm{z}$ directions. The resulting $\mathrm{y}$ perturbation was the product of the $\mathrm{x}$ and $\mathrm{z}$ perturbations.

$$
y=5.0+a\left[\sum_{i=1,4} A_{i} \cos \left(\frac{2 \pi x}{L_{i}}+\varphi_{i}\right)\right]\left[\sum_{i=5,8} A_{i} \cos \left(\frac{2 \pi z}{L_{i}}+\varphi_{i}\right)\right]
$$

The overall amplitude multiplier $a$ was set to 10.0. 
Table 3: component modes in initial material interface

\begin{tabular}{|l|c|l|l|}
\hline \multicolumn{1}{|c|}{$\mathrm{i}$} & \multicolumn{1}{c|}{$A_{i}$} & \multicolumn{1}{c|}{$L_{i}$} & \multicolumn{1}{c|}{$\varphi_{i}$} \\
\hline \hline 1 & $2.533581893 \mathrm{e}-02$ & 2.0 & $\pi / 2$ \\
\hline 2 & $9.346853101 \mathrm{e}-03$ & 3.0 & 0 \\
\hline 3 & $6.084968907 \mathrm{e}-02$ & 4.0 & $\pi / 2$ \\
\hline 4 & $9.034202601 \mathrm{e}-02$ & 5.0 & 0 \\
\hline 5 & $1.958731928 \mathrm{e}-02$ & 2.0 & $\pi / 2$ \\
\hline 6 & $4.629535430 \mathrm{e}-02$ & 3.0 & 0 \\
\hline 7 & $9.390213302 \mathrm{e}-02$ & 4.0 & $\pi / 2$ \\
\hline 8 & $1.272157551 \mathrm{e}-02$ & 5.0 & 0 \\
\hline
\end{tabular}

\subsection{Cloutman model effects on the test problem}

The effects of LES were mostly noticeable at small scales. Small scale mixing of momentum and internal energy were increased by the subgrid scale model. However, the quantities measured in this study were large scale: the growth of the entire instability and the balance of energy throughout the domain. Therefore, this is a study of the large scale effects of LES.

The Cloutman model creates a subgrid scale kinetic energy field as well as an eddy viscosity. There are several constants which can be modified to illustrate the behavior of these parameters. The quantity of eddy viscosity is modified by A as shown in EQ 40 . As A goes to zero, the model is effectively shut off and $k$ goes to zero. The default value was $A=0.44 . A s \Lambda$ increased, the instability growth rate decreased due to increased viscosity. 


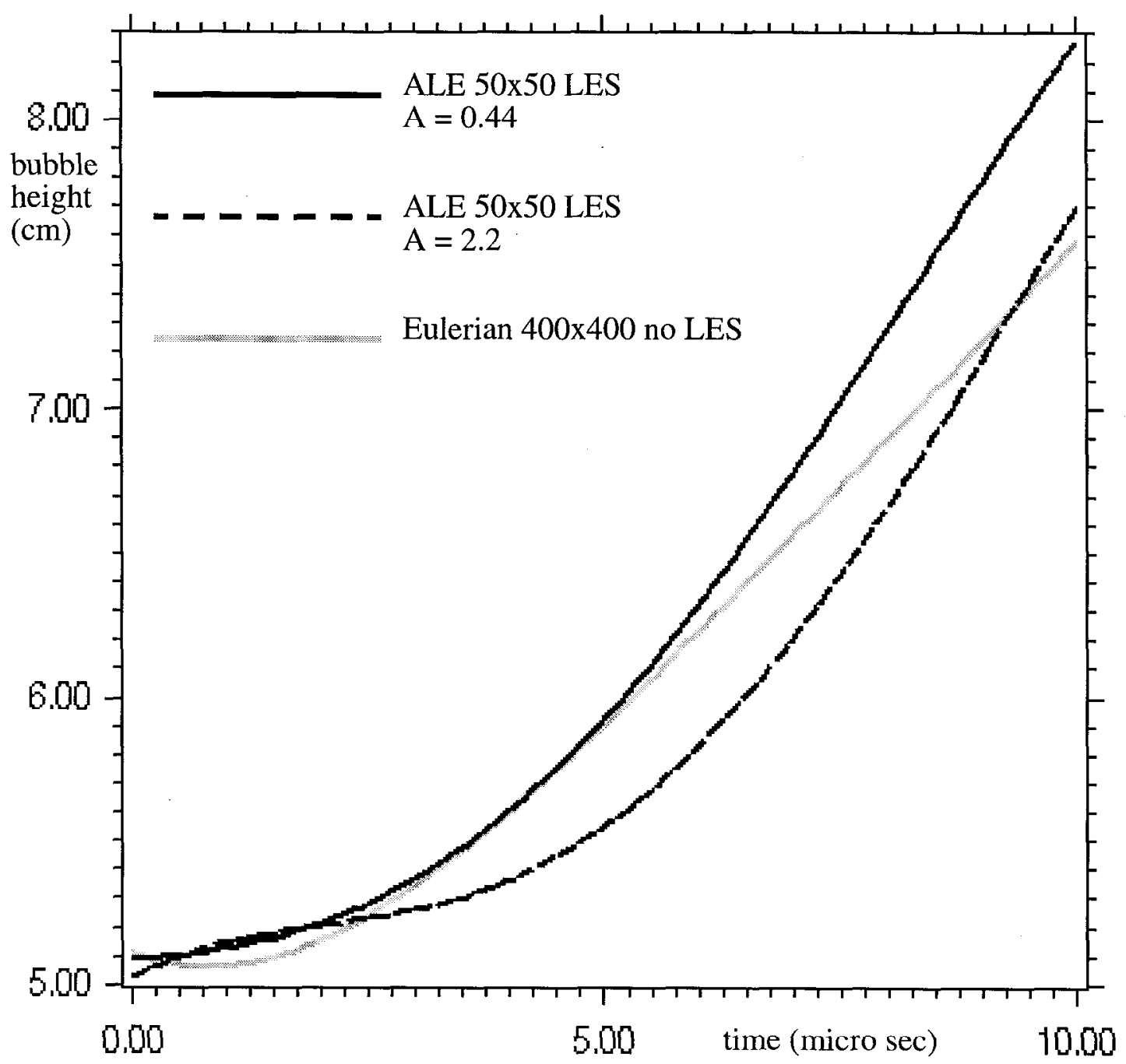

Fig. 22: Viscosity and growth rate in Cloutman model

It is impossible to decouple the effect of the eddy viscosity from the other parts of the model, since viscosity appears in the source term as well. Therefore, increasing A also decreased resolved kinetic energy, increased potential energy, increased subgrid scale kinetic energy, and altered internal energy.

The source and sink terms also have constant factors which modify the amount of energy in the subgrid scale kinetic energy field and the internal energy field. The buoy- 
ancy source term in EQ 38 is modified by B. The default value if B was 1.0. Increasing $\mathrm{B}$ increased the amount of $k$ that was created, and decreased internal energy to compensate. Dissipation of $k$ into internal energy is modified by $\mathrm{D}$. The default value of $\mathrm{D}$ was 0.37. Increasing $\mathrm{D}$ increased the amount of $k$ that was dissipated into internal energy. It is interesting to note that internal energy first decreases, then increases in this test problem. This is probably due to the material hitting the wall at the top of the domain.

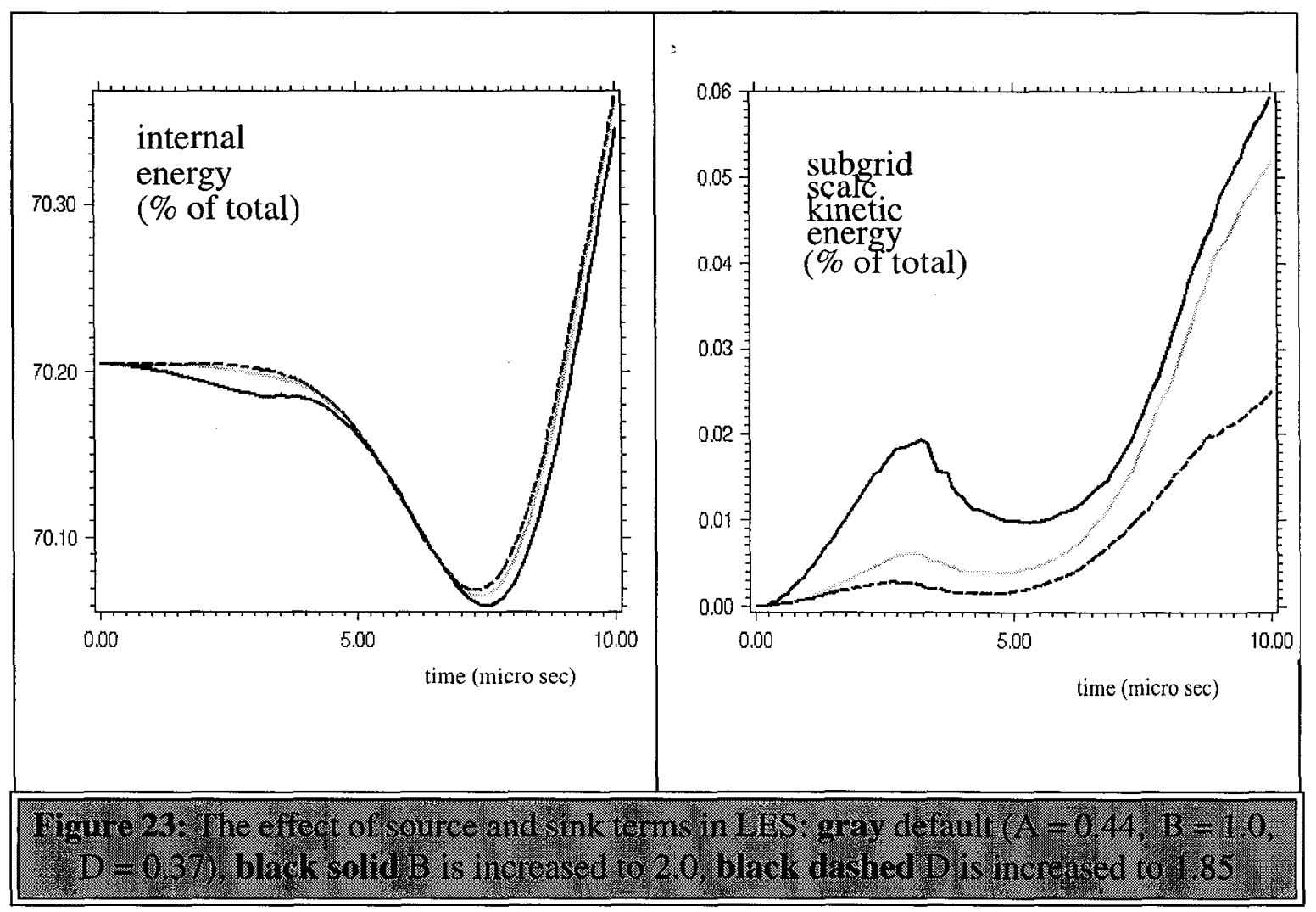

The parameters $\mathrm{A}, \mathrm{B}$, and $\mathrm{C}$ can be used to adjust instability growth and energy balance for more accurate simulations. However, eddy viscosity can only decrease instability growth. Stochastic backscatter is one way in which small scale motion can be added to the simulation to increase instability growth. 


\subsection{Stochastic backscatter effects}

The coupling of large scale interface instability modes usually slows overall instability growth, but the presence of additional small modes during the course of the simulation can speed growth. Stochastic backscatter serves this purpose, by adding a random component to the velocity at each time step.

Stochastic backscalter couples the subgrid scale kinetic energy with the large scale kinetic energy, and allows what amounts to viscous cooling. The presence of backscatter reduced the amount of subgrid scale kinetic energy (see Fig. 24 ), because the random velocity term acts as a dissipation term in the $\mathrm{k}$ equation. This dissipation of energy is not returned to the internal energy equation, however, since the viscous heating term due to molecular dissipation in the internal energy equation is neglected. 


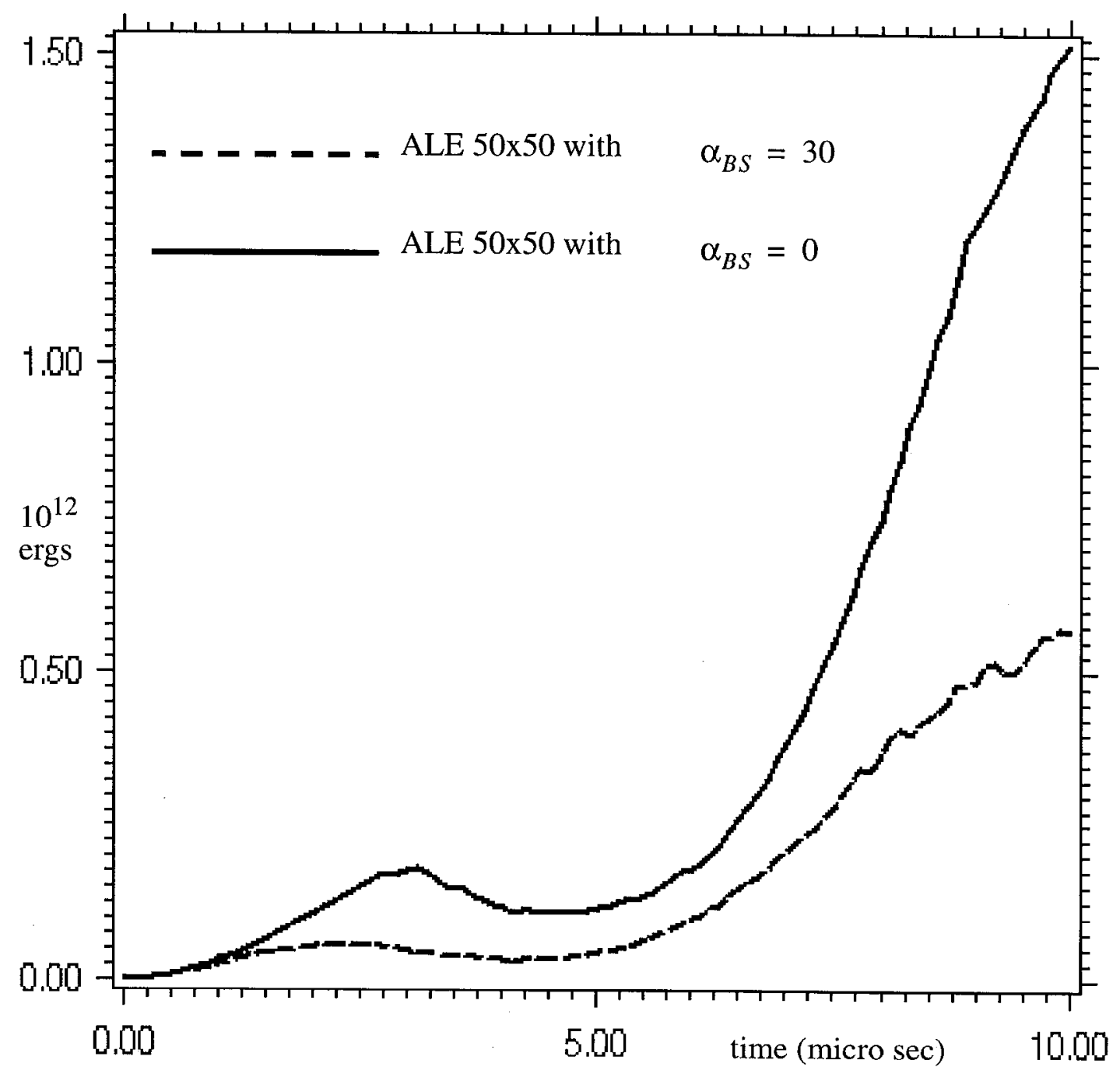

Fig. 24: Subgrid scale kinetic energy and stochastic backscatter

Stochastic backscatter caused a slight increase in overall instability growth, as can be seen in Fig. 25. This is due to the addition of small scale modes to the instability, and the reduction in $\mathrm{k}$ which leads to a reduction in the eddy viscosity. Little difference in the shape of the bubbles and spikes could be observed due to the random element of the velocity in this test problem. The effect of stochastic backscatter was so slight that a large value of $\alpha_{B S}=30$ was needed to see it. 


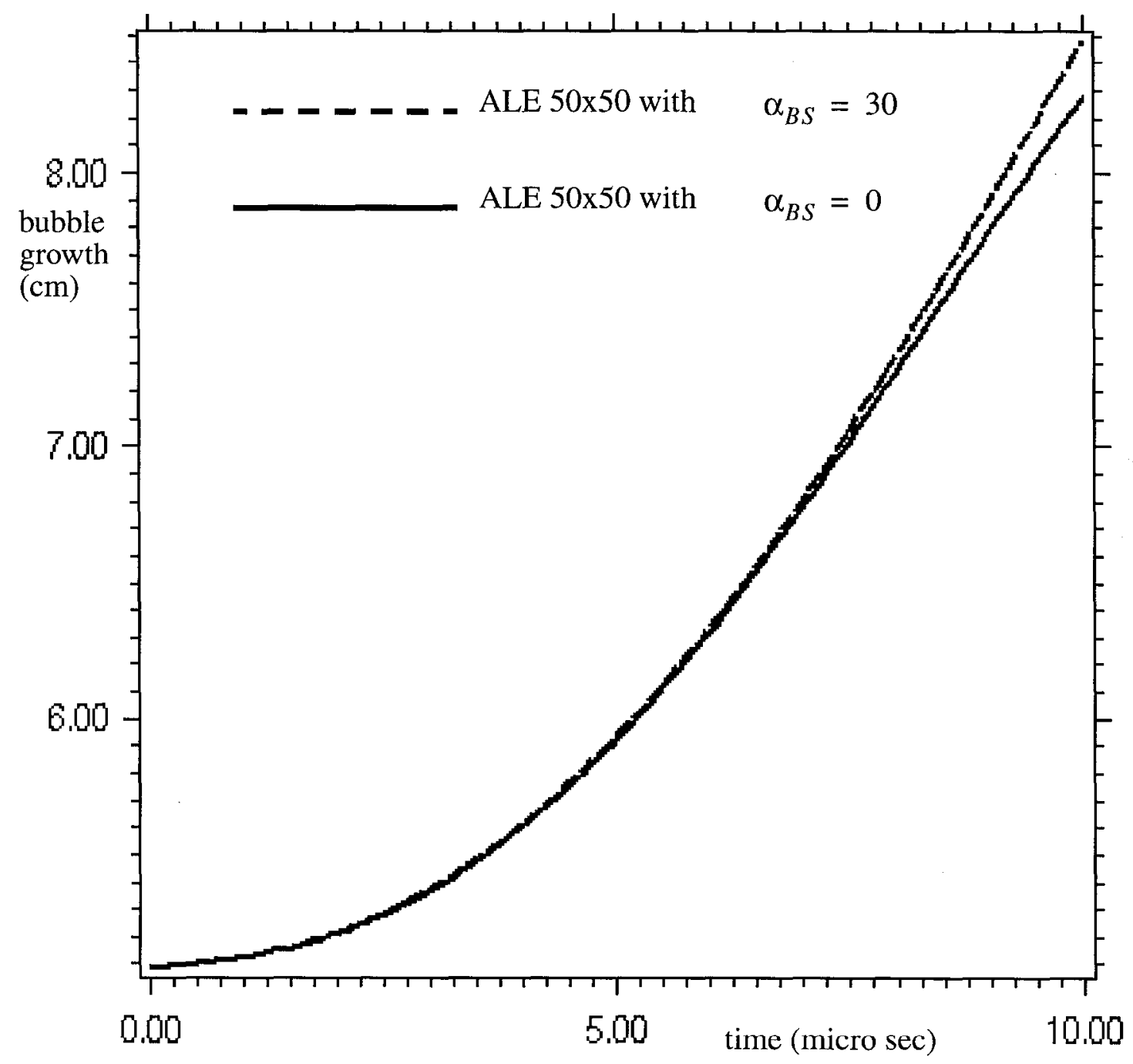

Fig. 25: The effect of backscatter on bubble growth

Early in the simulation, the backscatter velocity perturbation was drowned by a velocity caused by diffusion. The $k$ field first appeared next to the material interface.

The turbulent pressure $p_{t}=\frac{2}{3} \tilde{\rho} k$ has the same form as the non turbulent pressure $p=\frac{2}{3} \tilde{\rho} e$ as long as $\gamma=\frac{5}{3}$ in the ideal gas law for the problem. Since the unfiltered internal energy $e=\bar{e}+k$ the pressure will balance gravity as long as $k$ does not move 
between zones. But diffusion moves $k$ between zones, causing an imbalance at the interface. This caused a velocity along the interface which drowned the stochastic velocity. The presence of random terms in other variables might improve the effect of this stochastic backscatter model.

\subsection{Three Dimensions}

In general, 3 dimensional simulations of the test problem grew faster than 2 dimensional simulations. The viscosity model slowed the growth of the 3 dimensional simulation just as it did for the 2 dimensional simulation. 


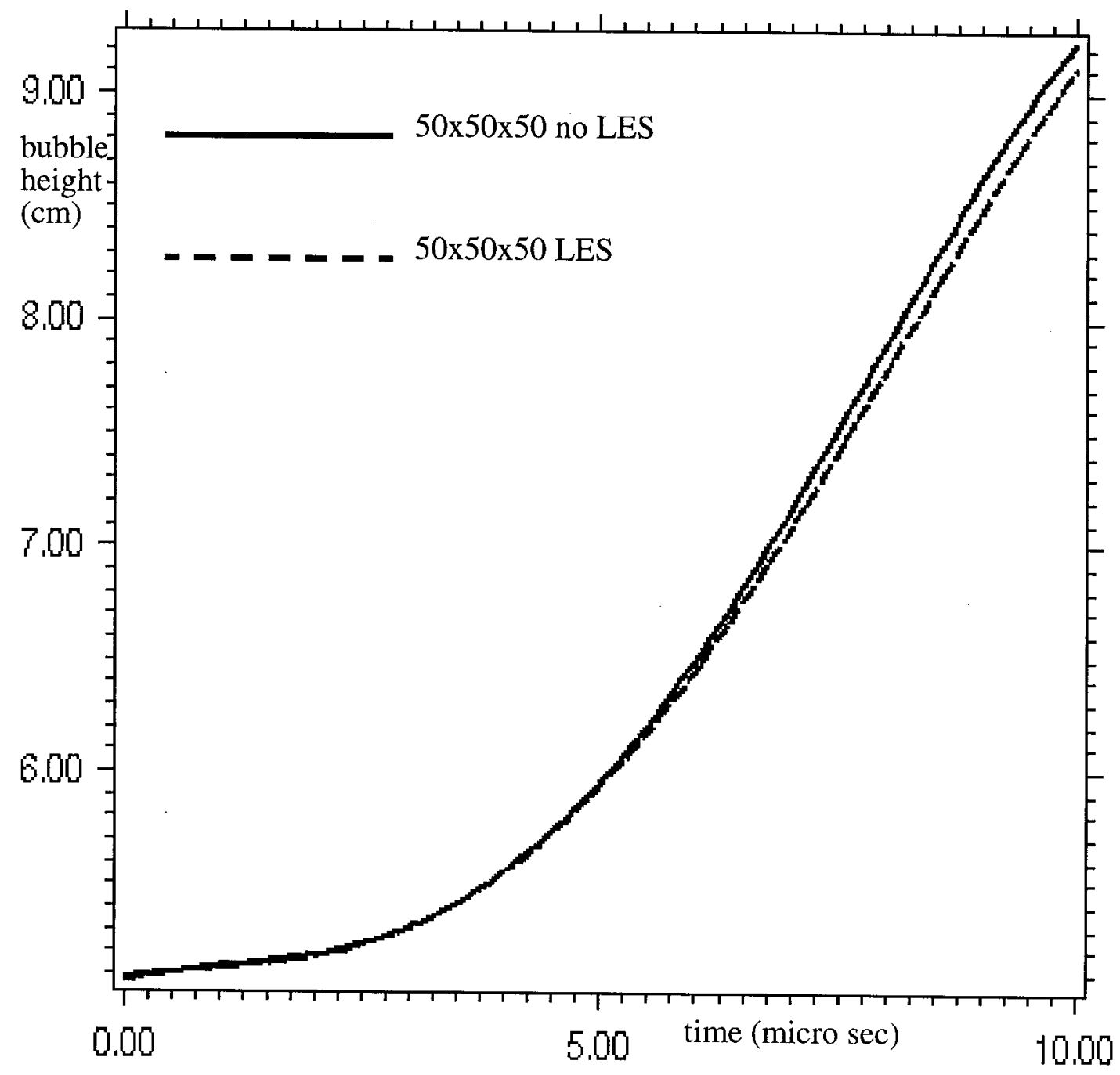

Fig. 26: 3 dimensional LES

The LES model with stochastic backscatter increased instability growth in 3 dimensions as well as 2 dimensions, but had a much greater effect. 


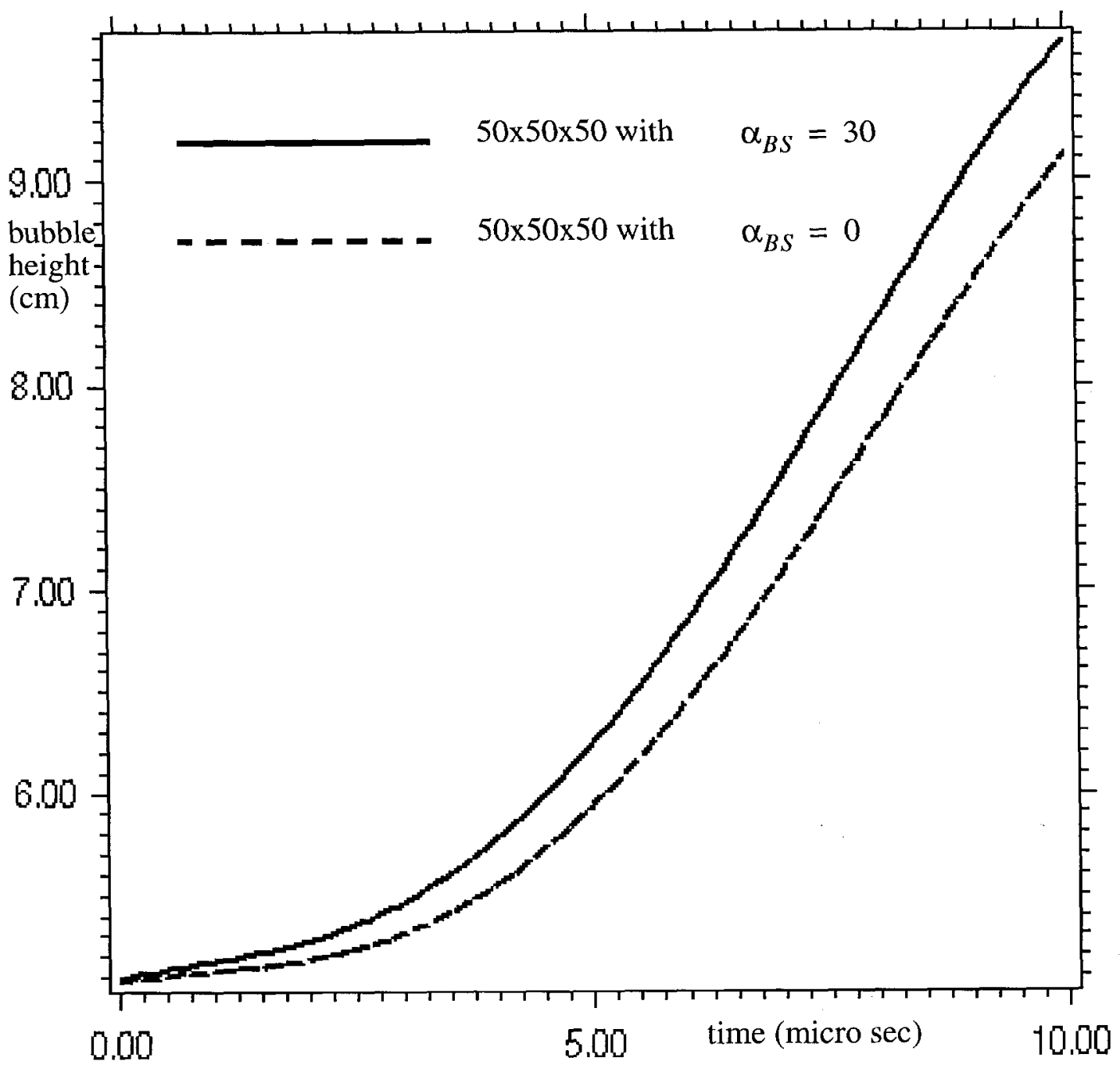

Fig. 27: 3 dimensional backscatter

\subsection{ALE mesh motion and LES}

LES has typically been developed for Eulerian meshes. As the ALE mesh moves, it changes resolution locally. The filter function used to separate resolved and unresolved motion and energy varies in space and time.

The computational solution of the filtered equations of motion assumes a uniform definition for the large scale and subgrid scale throughout the domain. In order to 
approximate the gradients of large scale variables across neighboring zones, the variables in each zone must represent the same quantity. However, as the mesh becomes non-uniform, each zone's large scale is defined according to the local grid scale. The large scale variables in neighboring zones represent different quantities.

However, ALE mesh motion is designed to minimize grid distortion. If neighboring zones are very uneven, the zones will be remapped to make them more even. This is intended to make the finite volume derivatives more accurate. The derivatives of zone centered quantities are located at the nodes, and the derivatives of node centered quantities are located at the zone centers. This assumption is only accurate if zones are evenly spaced, so each node is in the center of its neighboring zones.

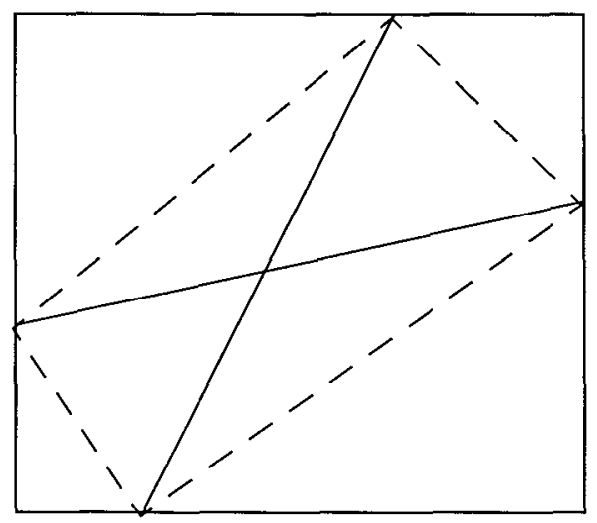

A node is remapped if:

- area of one triangle surrounding the zone is much smaller than others

- angle of any zone is too small

Fig. 28: Remap conditions

In the Cloutman model, subgrid scale kinetic energy is created locally in areas where change is taking place in the energy field. In the case of Rayleigh-Taylor instability simulations, this is at the interface between the two fluids, which is moving and twisting into convoluted shapes. This part of the mesh needs to remain closer to Eulerian than the rest of the mesh, in order to keep from tangling. So the use of a subgrid scale model designed 
for an Eulerian mesh may be appropriate.

The computational solution of the filtered equations of motion assumes a uniform definition for the large scale and subgrid scale in time as well as space. Values of variables at the previous time step are used to find updated values at the next time step. To quantify the effect of the moving mesh on the first order $k$ model, the rate of change of the $k$ field was compared to the rate of change of the zone length scale. Both fields were normalized to make them comparable quantities, so the plotted quantity is $\left|\frac{\partial}{\partial t} \ln (f)\right|$ where $f$ is either $k$ or $\lambda$. 


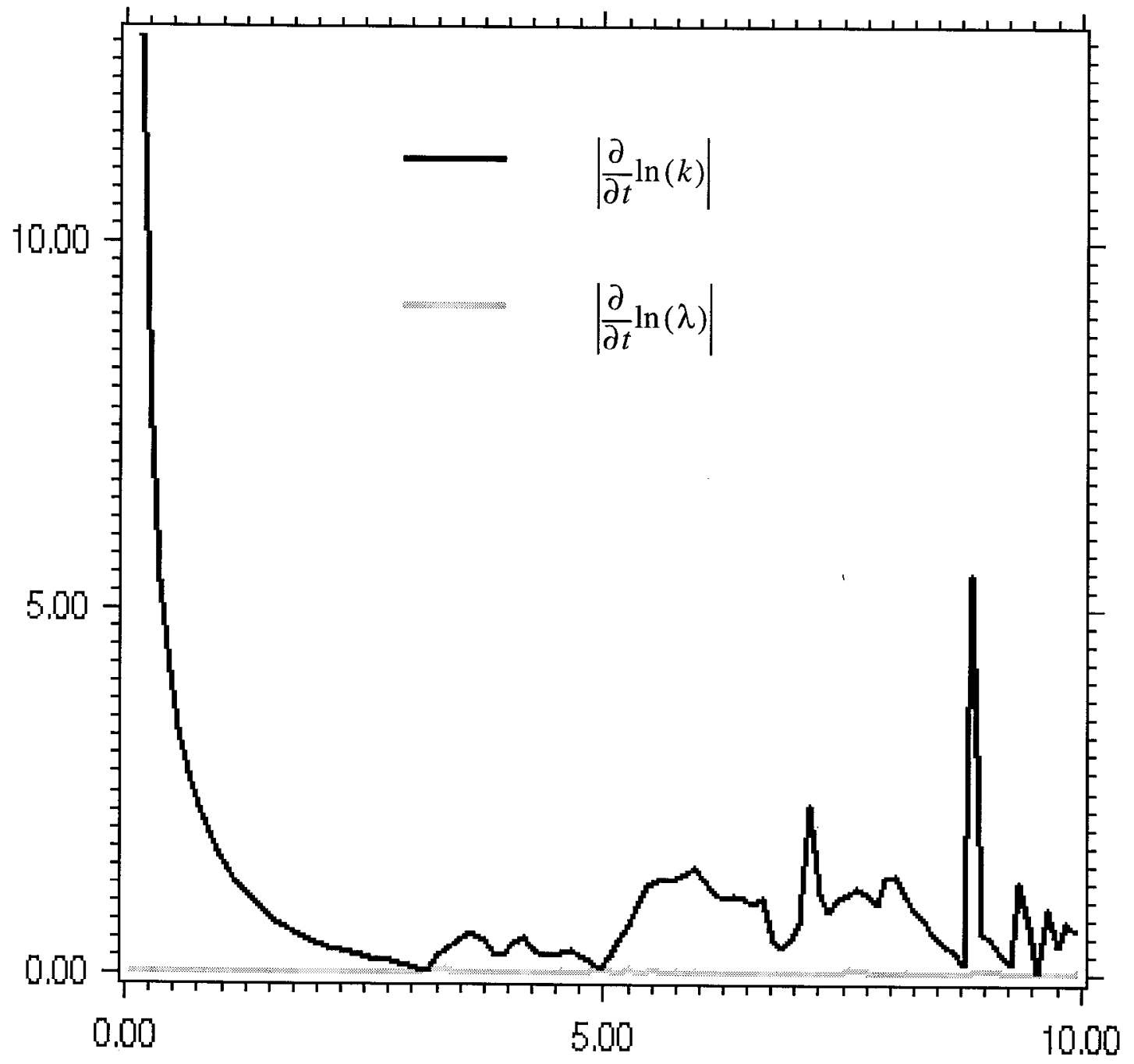

Fig. 29: Average of every fifth zone horizontally along midline 


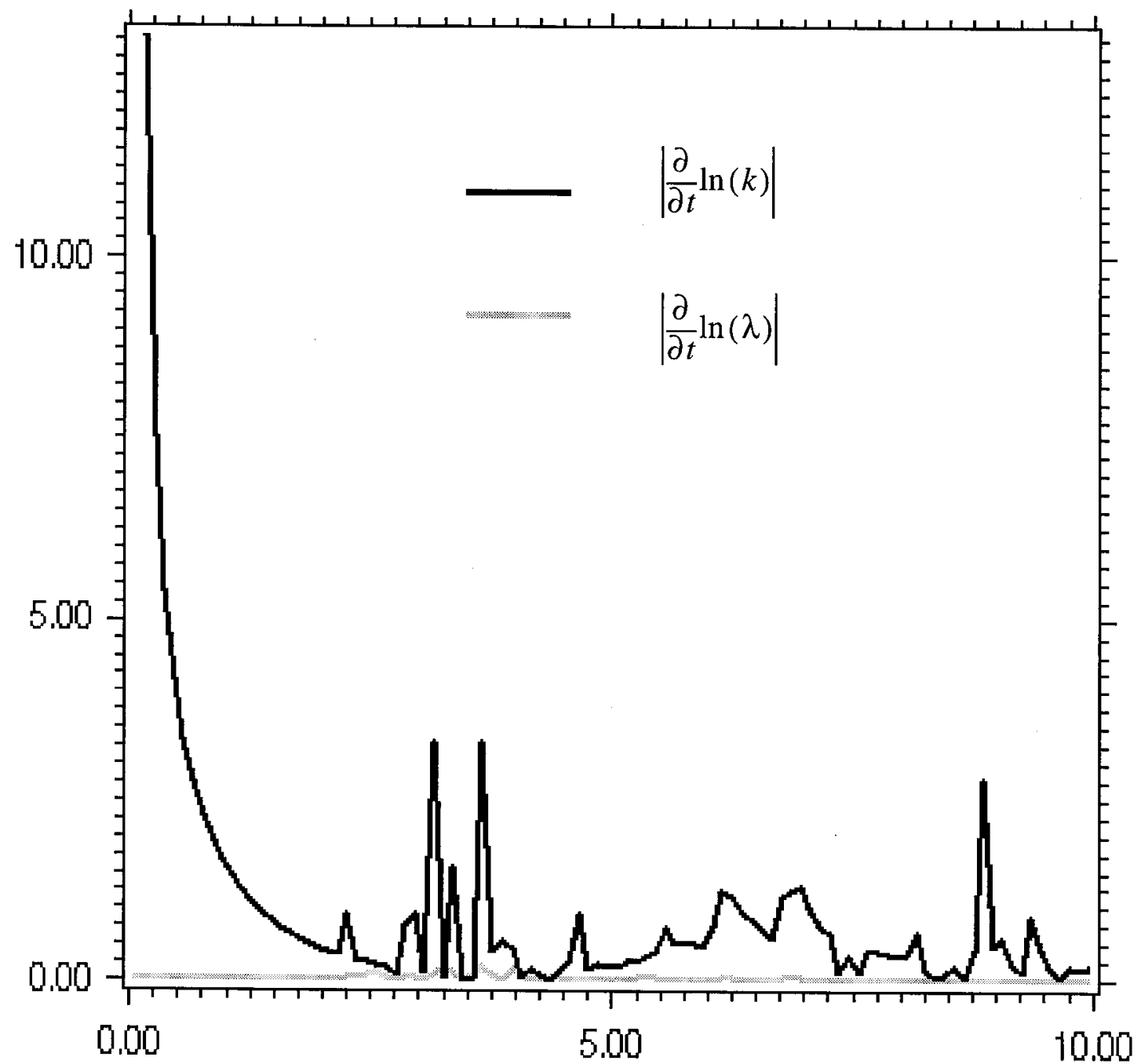

Fig. 30: Avcrage of every fifth zone vertically along midline

The averaged plots show that generally $k$ changed faster than the grid size, so the grid may appear stationary in comparison with the $k$ model evolution.

The effect of the moving mesh on LES has not been eliminated, but in this case it may be minimized since the changes are gradual. Spatial variations in the grid are minimized, and temporal changes appear to be small compared to the changes in $k$. 


\subsection{Subgrid scale kinetic energy as a grid relaxation weight}

The ALE method can be tied to the LES to improve the simulation in ways that are impossible for fixed mesh simulations. The grid relaxation component of ALE mesh motion allows for weights to be placed on particular areas of the mesh. Increasing the weight in one area causes mesh nodes to be drawn to this area more than other areas. Calculating weights from subgrid scale kinetic energy can draw the mesh into areas where unresolved energy is highest. This can improve the resolution of the problem where it is needed. The disadvantage is that making the grid less uniform reduces the accuracy of the large scale derivatives and the subgrid scale model as well.

In this study, a grid relaxation weight was created whose magnitude depended on the magnitude of $k$ in the zone to a certain degree, within a maximum limit placed on the weight. The function used to calculate the weight factor is

$$
\text { weightfactor }=\frac{\left(m k+k^{\prime}\right)}{\left(k+k^{\prime}\right)}
$$

where $m$ is the maximum weight and $k^{\prime}$ represents a typical value of $k$. The value of $m$ was 4, since Tipton [63] found that weights based on physical parameters were harmful if larger. As $k \ll k^{\prime}$ the weight factor goes to 1.0 , which eliminates it's effect. As $k » k^{\prime}$ the weight factor goes to the maximum $m$.

The choice of $k^{\prime}$ alters the effectiveness of the weight. Initially $k$ is zero everywhere, and remains zero through most of the mesh during the simulation. But in the area of the interface, $k$ grows. For this problem, the maximum zonal $k$ was about 0.2 at $50 \times 50$ resolution. If $k^{\prime}$ is too small, zones which contain $k$ form a block of high weight zones. If 
$k^{\prime}$ is too large, the weight has no effect since all weights are about 1.0. The most effective value for $k^{\prime}$ was found to be about 0.1 in this case. Replacing the constant value for $k^{\prime}$ with a value based on a local average of $k$ would probably make the weight factor more effective. The goal is to create variation in neighboring zonal weights. A difference in the relative weight of two neighboring zones alters mesh motion, rather than the value of any individual zone's weight.

The $k$ weight caused zones to cluster in areas of high $k$, which in turn reduced the amount of $k$ created. This caused a very slight reduction in the bubble growth.

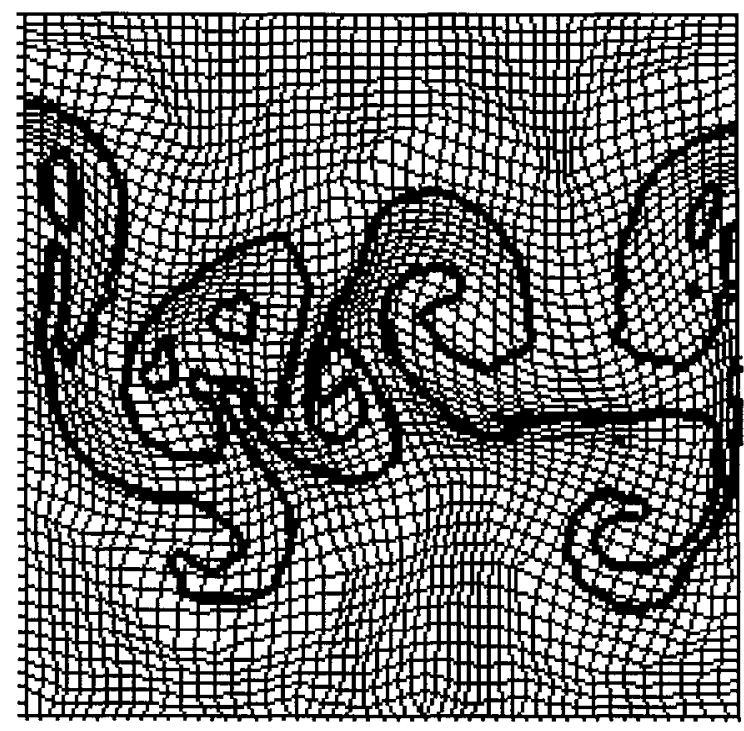

with $\mathrm{k}$ weight

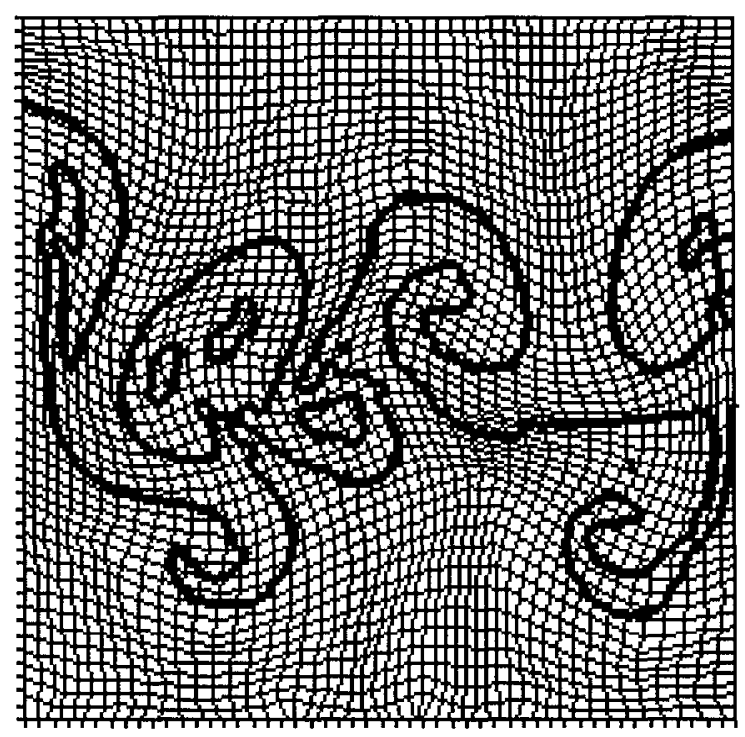

no k weight 


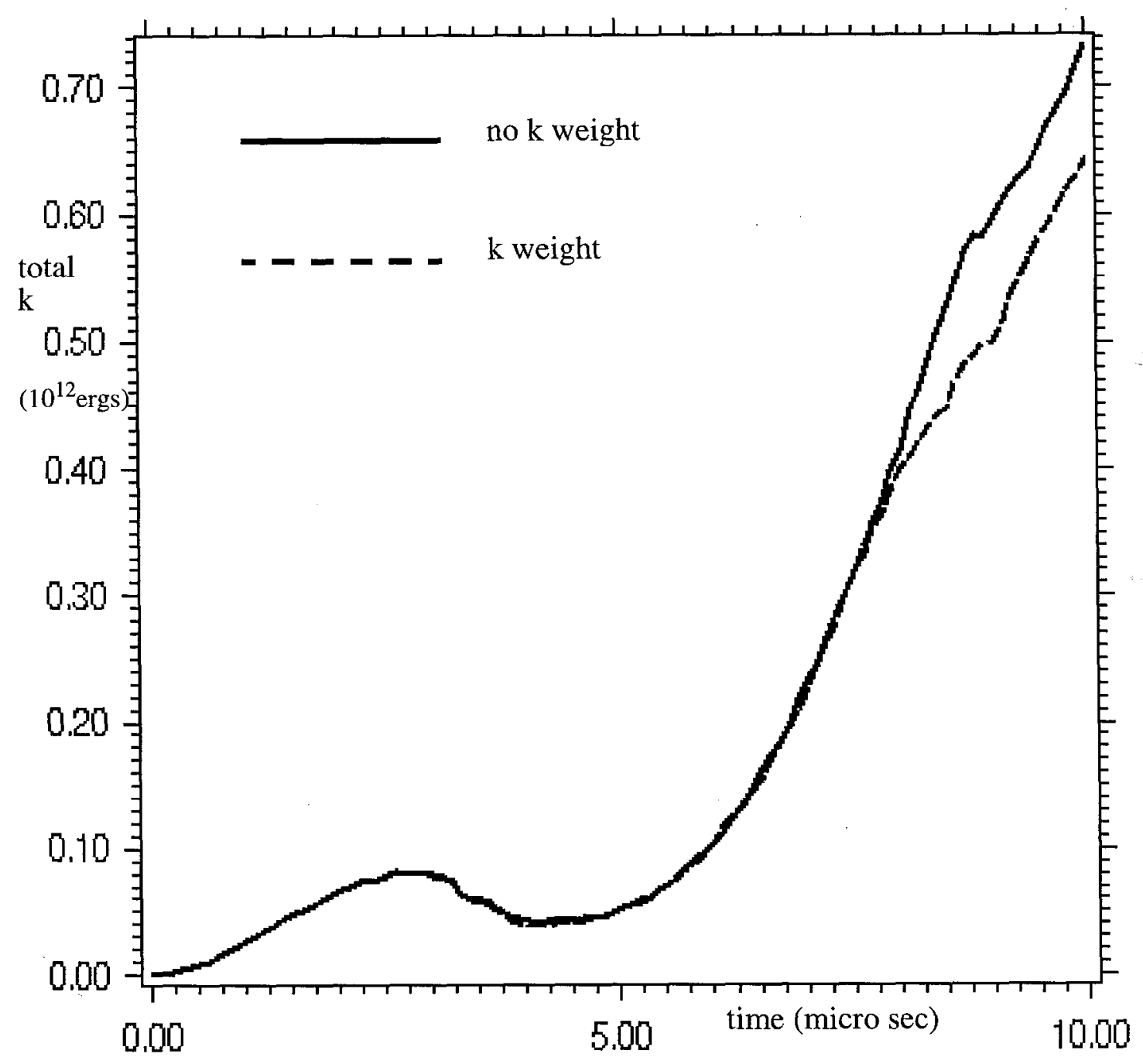

Fig. 31: Total turbulent kinetic energy and $\mathrm{k}$ weight

The difference between the minimum and maximum zonal length scale is an indication of how much extra grid distortion the $\mathrm{k}$ weight caused. Without the weight, the ratio of maximum to minimum length scale was 1.84 . With the $\mathrm{k}$ weight, this ratio increased to 2.59 . 
Table 4: min and max length scale

\begin{tabular}{|l|l|l|}
\hline & minimum length scale $(\mathrm{cm})$ & maximum length scale $(\mathrm{cm})$ \\
\hline \hline without $\mathrm{k}$ weight & $1.446746 \mathrm{e}-01$ & $2.667594 \mathrm{e}-01$ \\
\hline with $\mathrm{k}$ weight & $1.168927 \mathrm{e}-01$ & $3.025186 \mathrm{e}-01$ \\
\hline
\end{tabular}

The effect of the weight factor can be modified by averaging each zone's weight with the weights of neighboring zones, or replacing each zone's weight with the largest weight of its neighbors. Averaging weights locally had little effect on the effect of the weights, but replacing the weights reduced their effect.

\subsection{ALE vs. Eulerian LES}

The code in this study can be run Eulerian as well as ALE, by forcing the mesh to return to its original position after each Lagrangian step. Therefore, the behavior of the LES with ALE can be compared to its behavior in a fixed mesh. Generally, the two simulations were very similar. The largest difference was that the ALE simulation generated more subgrid scale kinetic energy than the Eulerian simulation. 


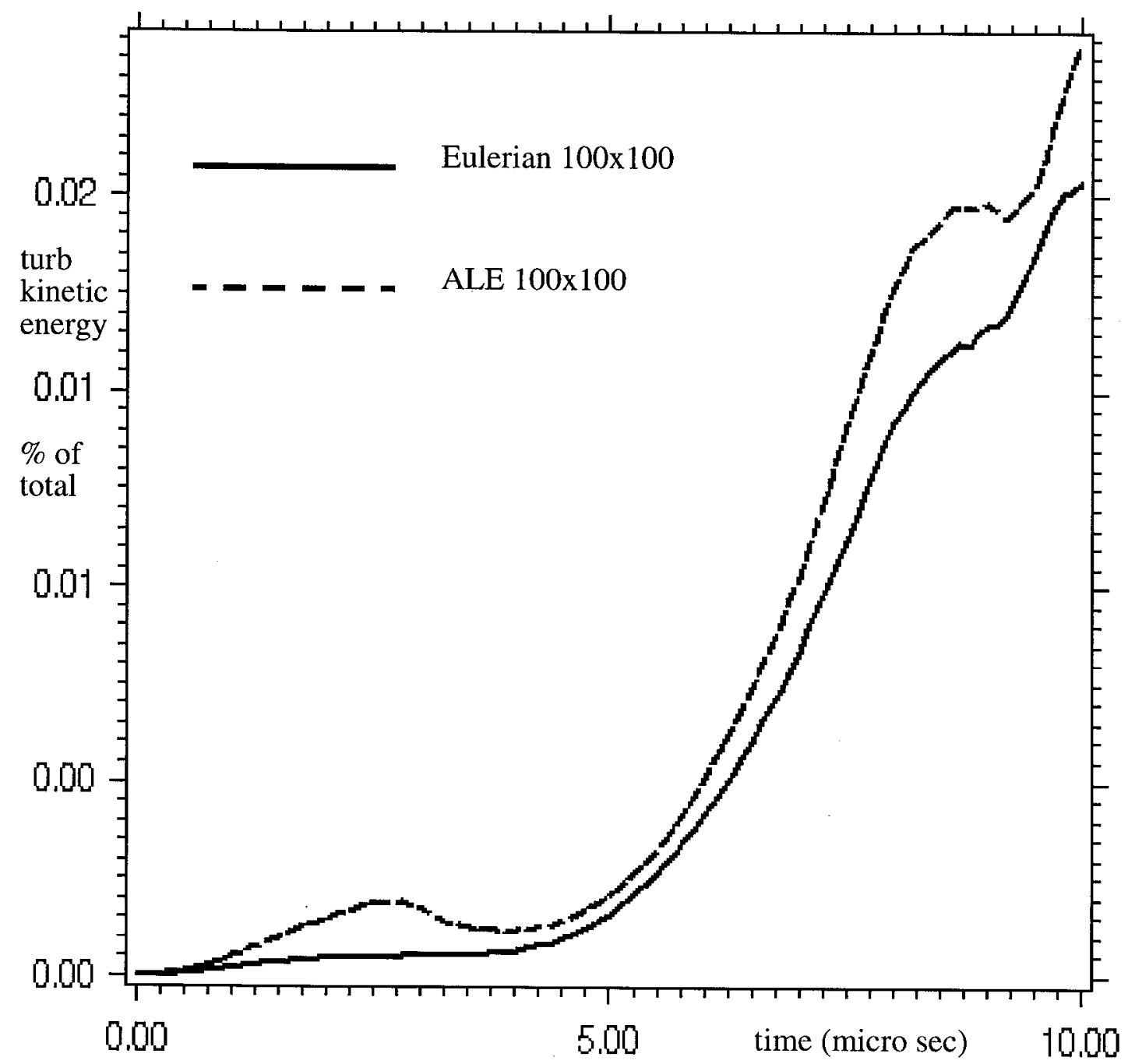

Fig. 32: Subgrid scale kinetic energy Eulerian vs. ALE

The rise in large scale kinetic energy and the fall in potential energy for the two simulations were very similar. The rise in internal energy was also greater for the ALE than 
the Eulerian mesh, but the difference was slight.

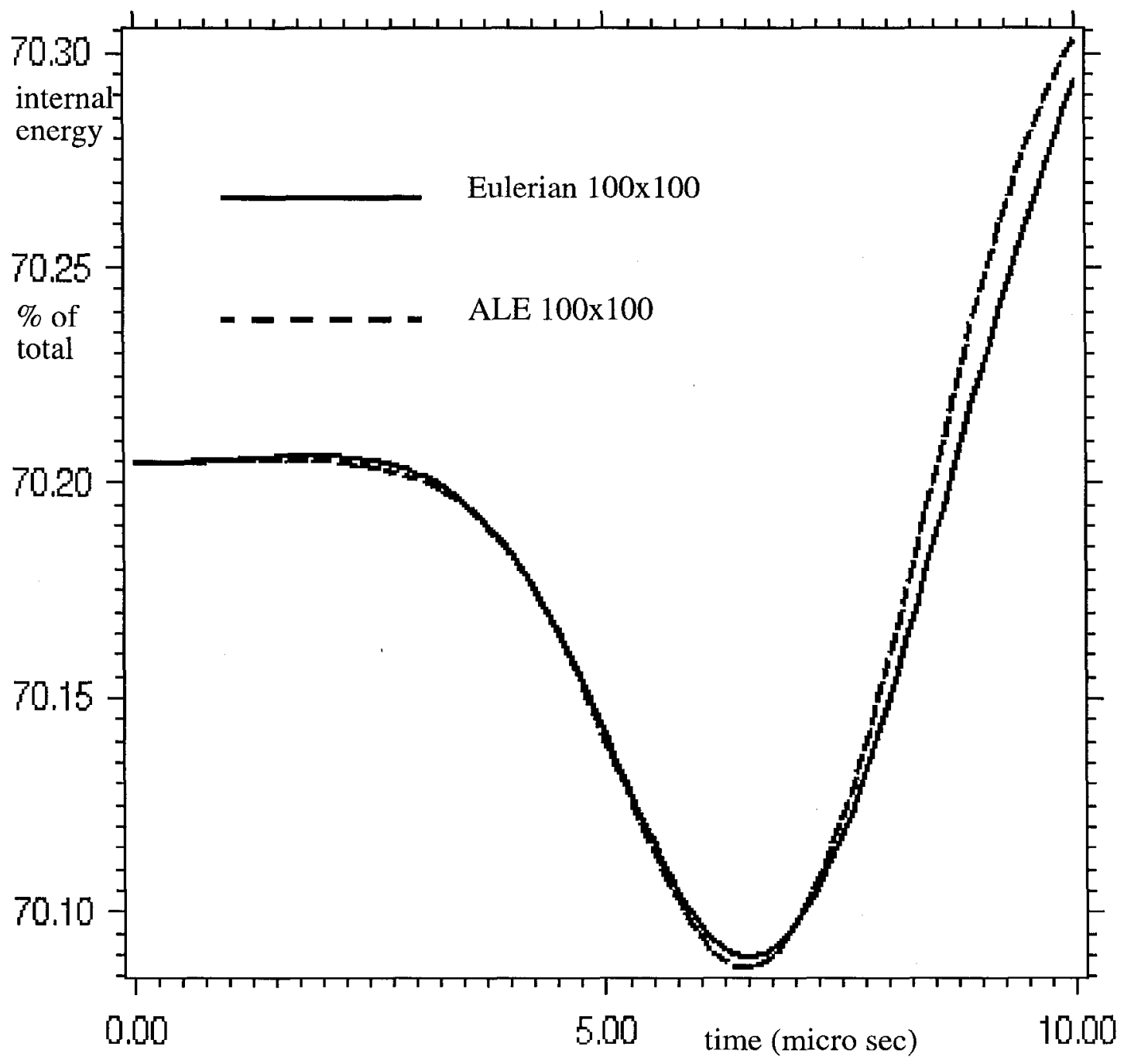

Fig. 33: ALE vs. Eulerian internal energy

In general, for this test problem, the behavior of LES was very similar for ALE and Eulerian simulations.

\subsection{Convergence study}

For both ALE and Eulerian simulations, increasing resolution decreased the amount of turbulent kinetic energy created, as can be seen in Fig. 34 and Fig. 35 . Convergence was calculated to be approximately first order. However, for the Eulerian simulation, the 
order of convergence was greater, with a lower standard deviation, than for the ALE simulation.

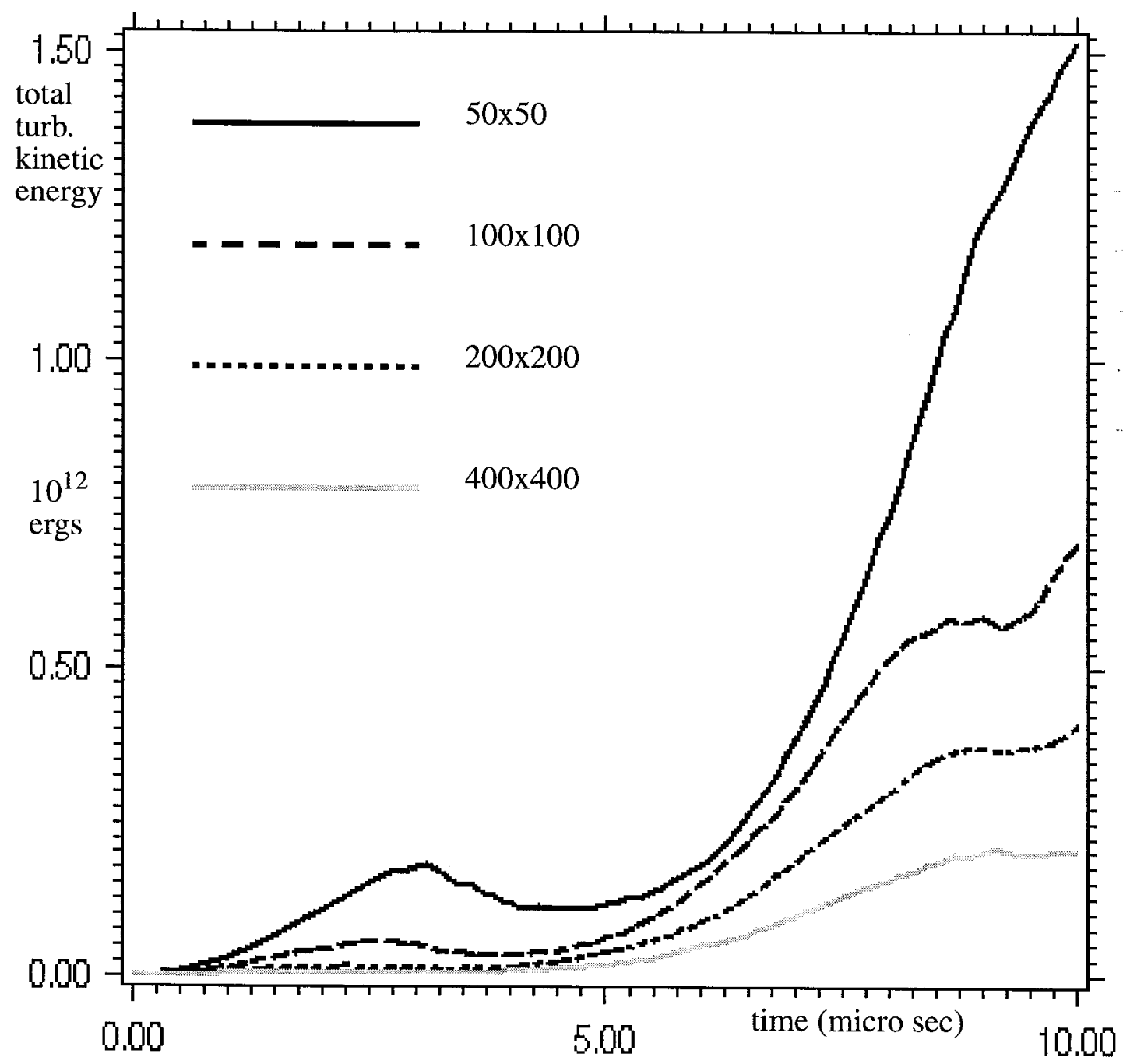

Fig. 34: ALE Turbulent kinetic energy and resolution

The power law relationship between subgrid scale kinetic energy and length scale is 
assumed to be $k=A \lambda^{n}$. The results are shown in Table 5 .

Table 5: ALE Convergence

\begin{tabular}{|l|l|l|}
\hline \multicolumn{1}{|c|}{ time } & \multicolumn{1}{c|}{ power $n$} & standard deviation \\
\hline \hline 1 & 1.172542 & 0.01264259 \\
\hline 2 & 1.609416 & 0.04751566 \\
\hline 3 & 1.892514 & 0.07942913 \\
\hline 4 & 1.321242 & 0.05099535 \\
\hline 5 & 0.8535244 & 0.04199536 \\
\hline 6 & 0.6496151 & 0.06010345 \\
\hline 7 & 0.6633421 & 0.1265966 \\
\hline 8 & 0.7556546 & 0.2596016 \\
\hline 9 & 0.8470779 & 0.4495634 \\
\hline 10 & 0.9485848 & 0.5776123 \\
\hline average: & $\mathbf{1 . 0 7 1 3 5 1}$ & $\mathbf{0 . 1 7 0 6 0 5 5}$ \\
\hline
\end{tabular}

Eulerian convergence was better than ALE convergence, but the difference was small. 


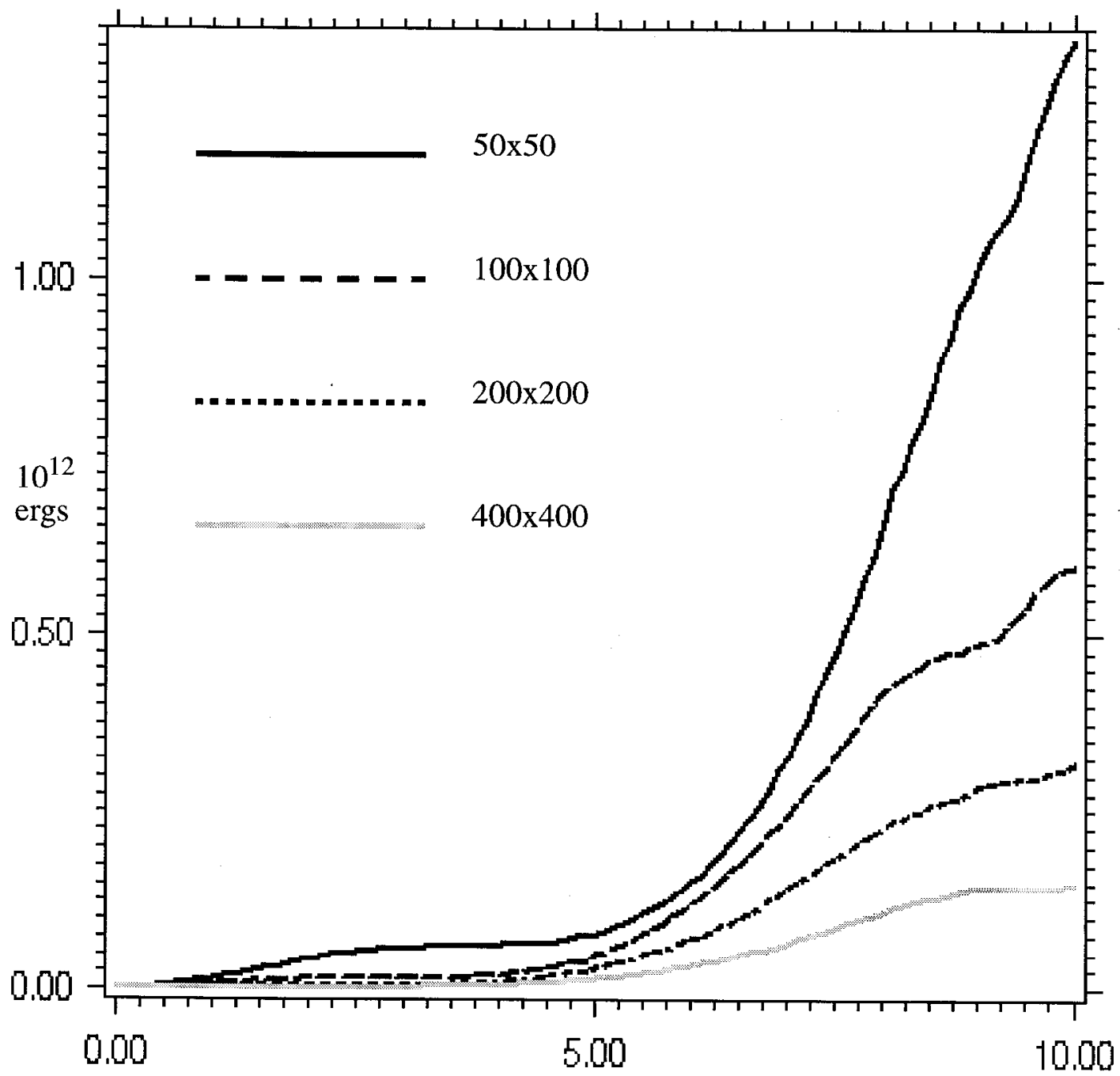

Fig. 35: Eulerian Turbulent Kinetic Energy and Convergence 
Table 6: Eulerian convergence

\begin{tabular}{|l|l|l|}
\hline \multicolumn{1}{|c|}{ time } & \multicolumn{1}{c|}{ power $n$} & standard deviation \\
\hline \hline 1 & 1.372085 & 0.005993379 \\
\hline 2 & 1.787583 & 0.01881907 \\
\hline 3 & 1.689841 & 0.02523898 \\
\hline 4 & 1.217672 & 0.02579273 \\
\hline 5 & 0.8339136 & 0.02747384 \\
\hline 6 & 0.7425943 & 0.05289287 \\
\hline 7 & 0.7869148 & 0.1169092 \\
\hline 8 & 0.8698476 & 0.2420375 \\
\hline 9 & 0.9352878 & 0.3868661 \\
\hline 10 & 1.053886 & 0.5259496 \\
\hline average: & $\mathbf{1 . 1 2 8 9 6 3}$ & $\mathbf{0 . 1 4 2 7 9 7 3}$ \\
\hline
\end{tabular}

\subsection{Conclusions}

The effect of ALE mesh motion on the performance of the Cloutman model was minimal in this test problem. The greatest difference was that ALE simulations created a larger amount of subgrid scale kinetic energy than Eulerian simulations. ALE is designed in such a way that it minimizes errors in spatial and temporal derivatives that might effect LES. Neighboring zones are kept close to the same size, which aids the accuracy of filtering large from small scales. The change in subgrid scale kinetic energy was greater than the change in grid size over time, so LES should not be greatly effected by the temporal changes in the grid in this case.

LES with ALE had a slightly lower convergence rate when compared to Eulerian 
simulations. But the LES could be directly tied to ALE mesh motion in the form of a grid relaxation weight. Grid resolution was increased in areas of unresolved energy, thus reducing the amount of unresolved energy. This is not possible with LES in an Eulerian mesh.

This study raises several questions for future research in LES with ALE. The test problem in this chapter involves minimal mesh motion. Simulations where greater ALE mesh motion occurs might make problems more obvious. Also, an unresolved issue remains concerning the effect of the subgrid scale model as material moves across the mesh from areas of greater resolution to areas of lower resolution. The process of filtering is effected by spatial and temporal changes in the mesh. A length scale model would partially decouple the subgrid scale model from the mesh, and connect subgrid scale energy more directly with the flow physics. This would complicate the connection between subgrid scale energy and mesh motion even further. 


\section{Simulations of Experiments}

The previous chapters examined the use of the $\Lambda$ rbitrary Lagrangian-Eulerian (ALE) method and large eddy simulation (LES) in simplified simulations of Rayleigh-Taylor instability. This chapter applies the same techniques to simulations of Rayleigh-Taylor instability experiments. The purpose is to find ways in which ALE with LES can be put to practical use. In Section 16.0, experiments conducted with the Linear Electric Motor apparatus are studied. Several changes were made to the test problem of the previous chapter to make a better approximation to the experiment. In Section 17.0, laser driven Rayleigh-Taylor experiments are studied, which show the usefulness of ALE and LES in more complicated simulations.

\subsection{Simulation of experiments with the Linear Electric Motor}

Many simulations of Rayleigh-Taylor instability experiments have been published. Youngs [72] published simulations of experiments performed by Read [54] in 1984. He measured the instability growth rate for comparison with experiment. He assumed that after the nonlinear phase (see Appendix A), Rayleigh-Taylor instability growth can be described by a similarity solution:

$$
\begin{aligned}
& \text { spike height } h_{1}=\alpha_{1} A g t^{2} \\
& \text { bubble height } h_{2}=\alpha_{2} A g t^{2}
\end{aligned}
$$

Over the years in various simulations, Youngs found the bubble growth $\alpha_{2}$ to have various values from $0.03-0.05[46][72][73][74][75]$. Others have found values for $\alpha_{2}$ as 
high as $0.06-0.07$ [21][27][44]. The current study focuses on the experimental work of Schneider et al. with the Linear Electric Motor apparatus [57].

The effect of Atwood number on Rayleigh-Taylor growth was investigated by Dimonte [16]. The experiments included density ratios $R$ between 1.3 and 50 for constant accelerations. The size of the fluid cavity and the magnitude of the acceleration

were varied to accommodate bubble and spike growth. Bubble growth $h_{2}=\alpha_{2} g t^{2}$ and spike growth $h_{1}=\alpha_{1} g t^{2}$ were found. Spike growth was assumed to fit a power law $\alpha_{1} \sim R^{D_{\alpha}}$

The Atwood number dependence of Rayleigh-Taylor growth has been studied numerically in several publications. Freed et al. [21] found a value of $\alpha_{2}=0.057$ for three density ratios $2: 3,1: 3$ and $1: 20$, and found bubble and spike penetration to be related to the inverse square root of the density ratio. Alon et al. [1] found that Rayleigh-Taylor instability reaches a constant acceleration as the Atwood number approaches 1. Youngs [74] also compared Rayleigh-Taylor growth at density ratios of 1.5, 3.0, and 20.0 in 3 dimensions. He found $\alpha_{2}-0.4$ and $\alpha_{2}-0.5$, depending on the calculation technique.

\subsection{Simulation details}

The linear electric motor apparatus accelerates a container filled with two fluids of different densities in the downward direction. This causes the heavier fluid on the bottom to push into the lighter fluid on the top. The magnitude of the resultant acceleration was about 73 times gravity for constant acceleration experiments. 
Numerical simulations of these experiments require several approximations. An ideal gas equation of state was used, so that $p=(\gamma-1) \rho e$. The experiments were incompressible, but the simulations were compressible. In order to reduce the compressibility of the simulations, the ideal gas law was modified. instead of the typical value of $\gamma=\frac{5}{3}$, a value of $\gamma=\frac{11}{3}$ was used. This reduced the density variation, but did not eliminate it. The density at a point initially $0.6 \mathrm{~cm}$ above the interface can be seen in Fig. 36. Ringing indicates the passage of the material interface near the point of measurement. 


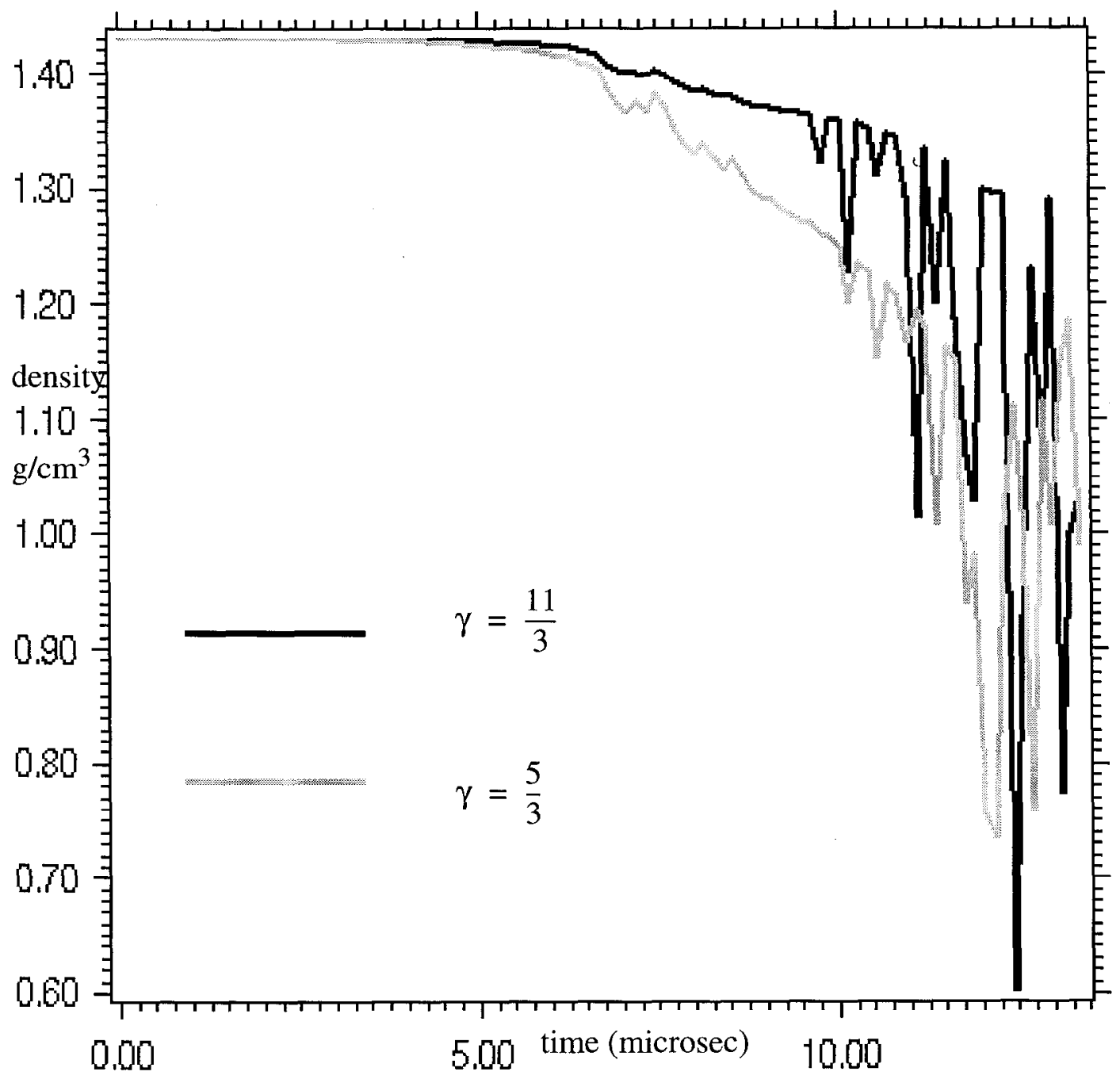

Fig. 36: Density variation at position $(3.5,5.0)$

The pressure at the top of the domain was set to atmospheric pressure, and the pressure gradient throughout rest of the domain was set to balance the acceleration $a$ such that $\nabla p=-\rho a$. This put the two materials in equilibrium initially.

In simulation units, the acceleration was $7.154 * 10^{-8} \mathrm{~cm} / \mu \mathrm{s}^{2}$, and the time for the experiment to run was $5^{*} 10^{5} \mu \mathrm{s}$. Using these values, the simulation could take about $10^{7}$ time steps. In order to reduce the number of time steps, $g t^{2}$ was treated as a con- 
stant. The simulation used an acceleration of $1 \mathrm{~cm} / \mu \mathrm{s}^{2}$ over a time of $13.37 \mu \mathrm{s}$, maintaining the value of $g t^{2}$ while reducing the number of time steps. To test the validity of this assumption, simulations were tried for various accelerations and times. The bubble growth rates were very similar for four orders of magnitude in the acceleration, as can be seen in Fig. 37 .

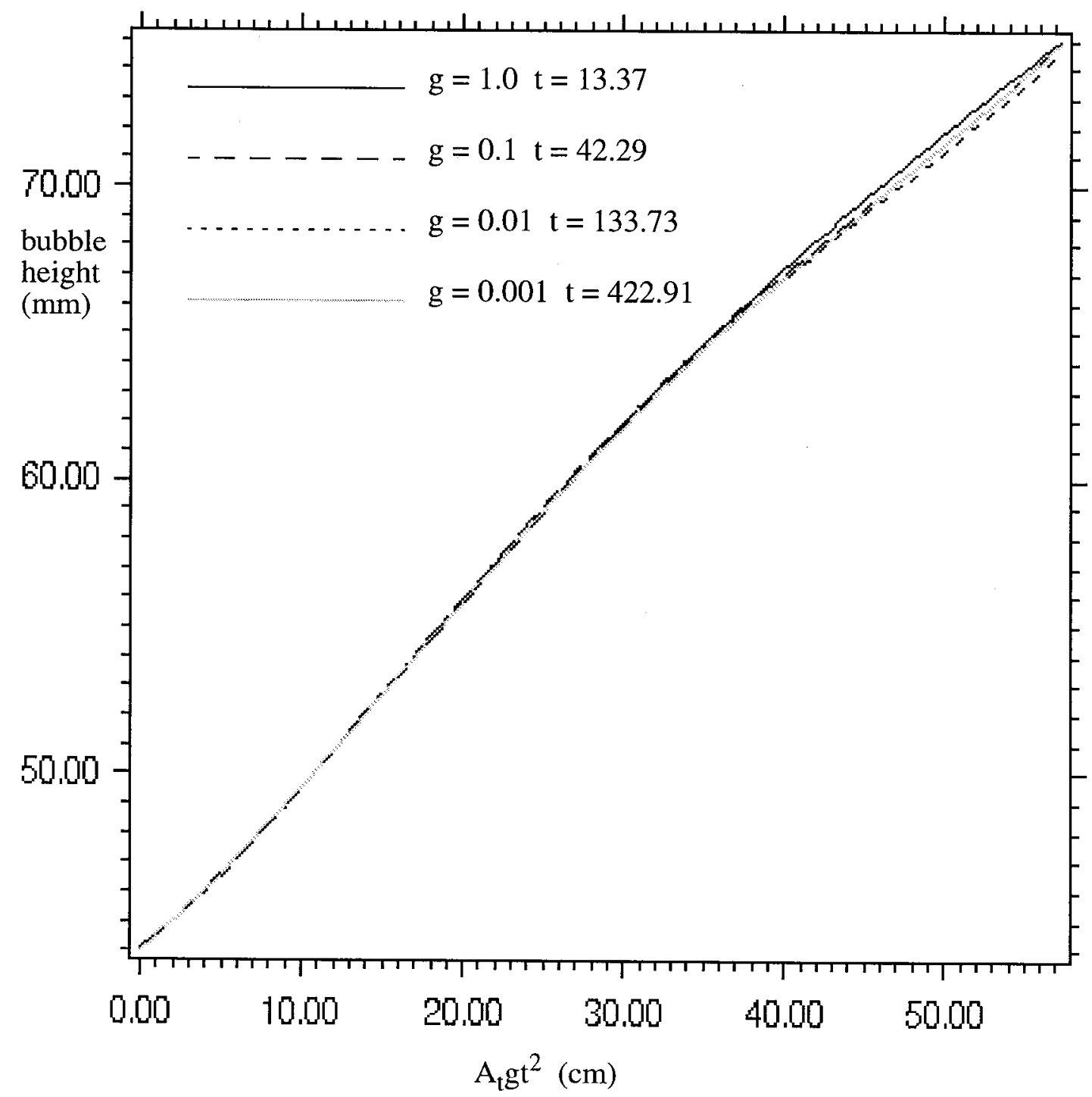

Fig. 37: Scaling by $\mathrm{gt}^{2}$

Two types of initial perturbation were examined: a random spatial perturbation and a 
multimode perturbation in which all constituent modes were resolved. The advantage of the multimode perturbation is that no initial modes are lost to insufficient horizontal resolution, so higher resolution simulations have the same initial perturbation as lower resolution simulations. In 2 dimensions, the initial perturbation at the fluid interface was a sum of four resolved modes with random amplitudes.

$$
y=0.1\left[\sum_{i=1,4} A_{i} \cos \left(\frac{2 \pi x}{L_{i}}+\varphi_{i}\right)\right]
$$

The values of the amplitudes, wavelengths, and phases are given in Table 7.

Table 7: component modes in initial material interface

\begin{tabular}{|l|c|l|l|}
\hline \multicolumn{1}{|c|}{$\mathrm{i}$} & \multicolumn{1}{c|}{$A_{i}$} & \multicolumn{1}{c|}{$L_{i}$} & \multicolumn{1}{c|}{$\varphi_{i}$} \\
\hline \hline 1 & $2.533581893 \mathrm{e}-02$ & 2.0 & $\pi / 2$ \\
\hline 2 & $9.346853101 \mathrm{e}-03$ & 3.0 & 0 \\
\hline 3 & $6.084968907 \mathrm{e}-02$ & 4.0 & $\pi / 2$ \\
\hline 4 & $9.034202601 \mathrm{e}-02$ & 5.0 & 0 \\
\hline
\end{tabular}

In 3 dimensions, the same four perturbation modes were created in the $\mathrm{x}$ and $\mathrm{z}$ directions, and 8 random amplitudes were chosen. In order to make the overall initial amplitude approximate that of the 2 dimensional perturbation, the overall multiplier was divided in half.

$$
y=0.05\left[\sum_{i=1,4} A_{i} \cos \left(\frac{2 \pi x}{L_{i}}+\varphi_{i}\right)+\sum_{i=5,8} A_{i} \cos \left(\frac{2 \pi z}{L_{i}}+\varphi_{i}\right)\right]
$$


Table 8: component modes in initial material interface

\begin{tabular}{|l|l|l|l|}
\hline \multicolumn{1}{|c|}{$\mathrm{i}$} & \multicolumn{1}{c|}{$A_{i}$} & \multicolumn{1}{c|}{$L_{i}$} & \multicolumn{1}{c|}{$\varphi_{i}$} \\
\hline 1 & $2.533581893 \mathrm{e}-02$ & 2.0 & $\pi / 2$ \\
\hline 2 & $9.346853101 \mathrm{e}-03$ & 3.0 & 0 \\
\hline 3 & $6.084968907 \mathrm{e}-02$ & 4.0 & $\pi / 2$ \\
\hline 4 & $9.034202601 \mathrm{e}-02$ & 5.0 & 0 \\
\hline 5 & $1.958731928 \mathrm{e}-02$ & 2.0 & $\pi / 2$ \\
\hline 6 & $4.629535430 \mathrm{e}-02$ & 3.0 & 0 \\
\hline 7 & $9.390213302 \mathrm{e}-02$ & 4.0 & $\pi / 2$ \\
\hline 8 & $1.272157551 \mathrm{e}-02$ & 5.0 & 0 \\
\hline
\end{tabular}

Varying the initial perturbation amplitude effected instability growth a great deal. This amplitude was adjusted for the most accurate growth in 2 dimensions. As the amplitude was reduced, the instability growth rate became smaller and smaller. This reflects the limited vertical resolution of the simulation. Numerical dissipation occurs for wavelengths that are too small to be captured by the grid, either in the horizontal or vertical direction.

The maximum sound speed for the 2 dimensional simulation was about $7 \mathrm{~cm} / \mu \mathrm{s}$. considering bubble growth to be about $0.22 \mathrm{~cm} / \mu s$, the mach number was roughly 0.03 .

\subsection{Simulation results}

An inviscid simulation in 3 dimensions with $50 \times 50 \times 50$ zones was compared with an inviscid simulation in 2 dimensions with $100 \times 100$ zones. Growth was faster in 3 dimensions than in 2. This is not surprising considering the findings of Hecht et al. [31], who observed that 3 dimensional simulations of single mode Rayleigh-Taylor instabilities 
grew further than the corresponding 2 dimensional modes.

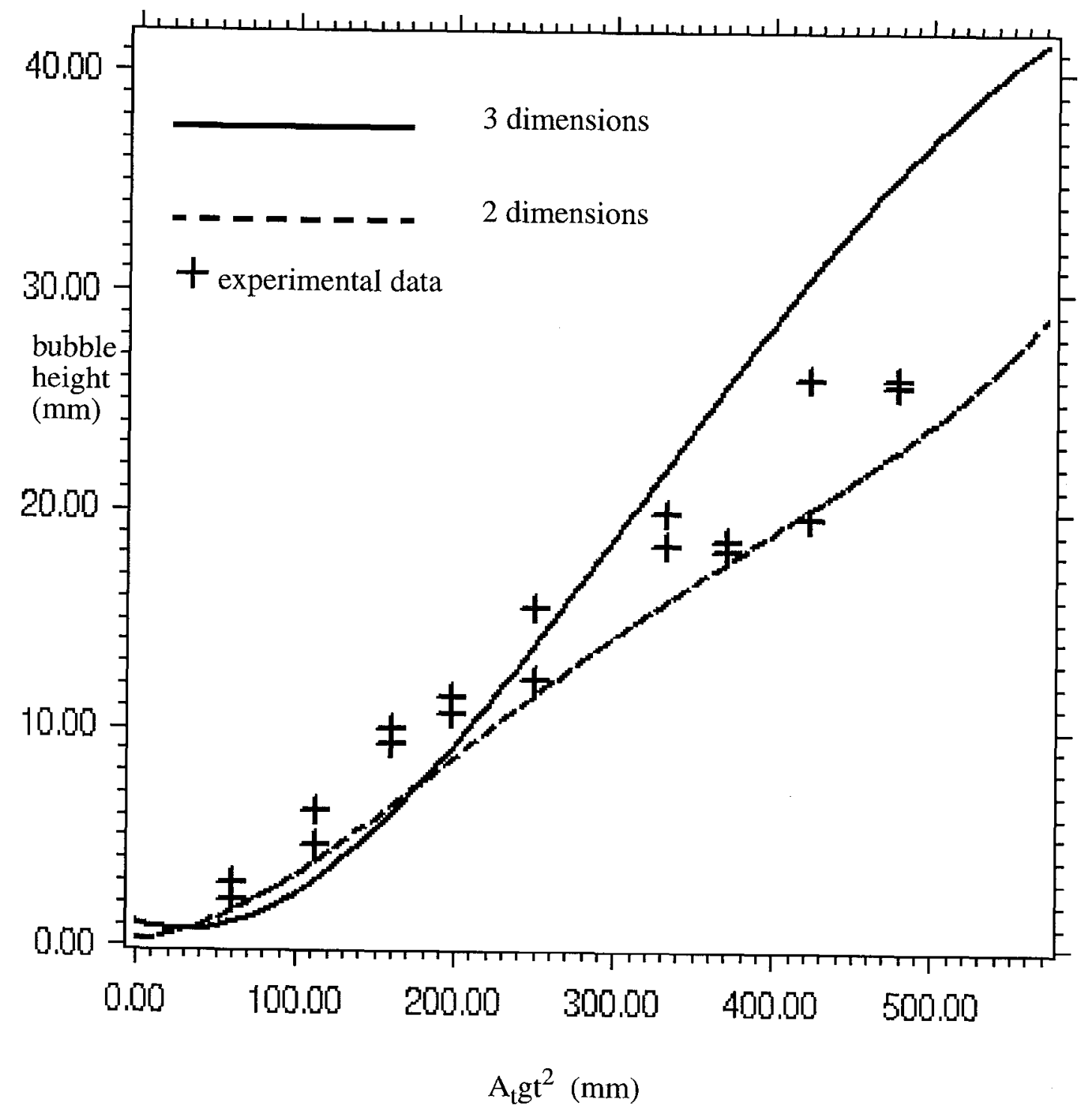

Fig. 38: Bubble growth 


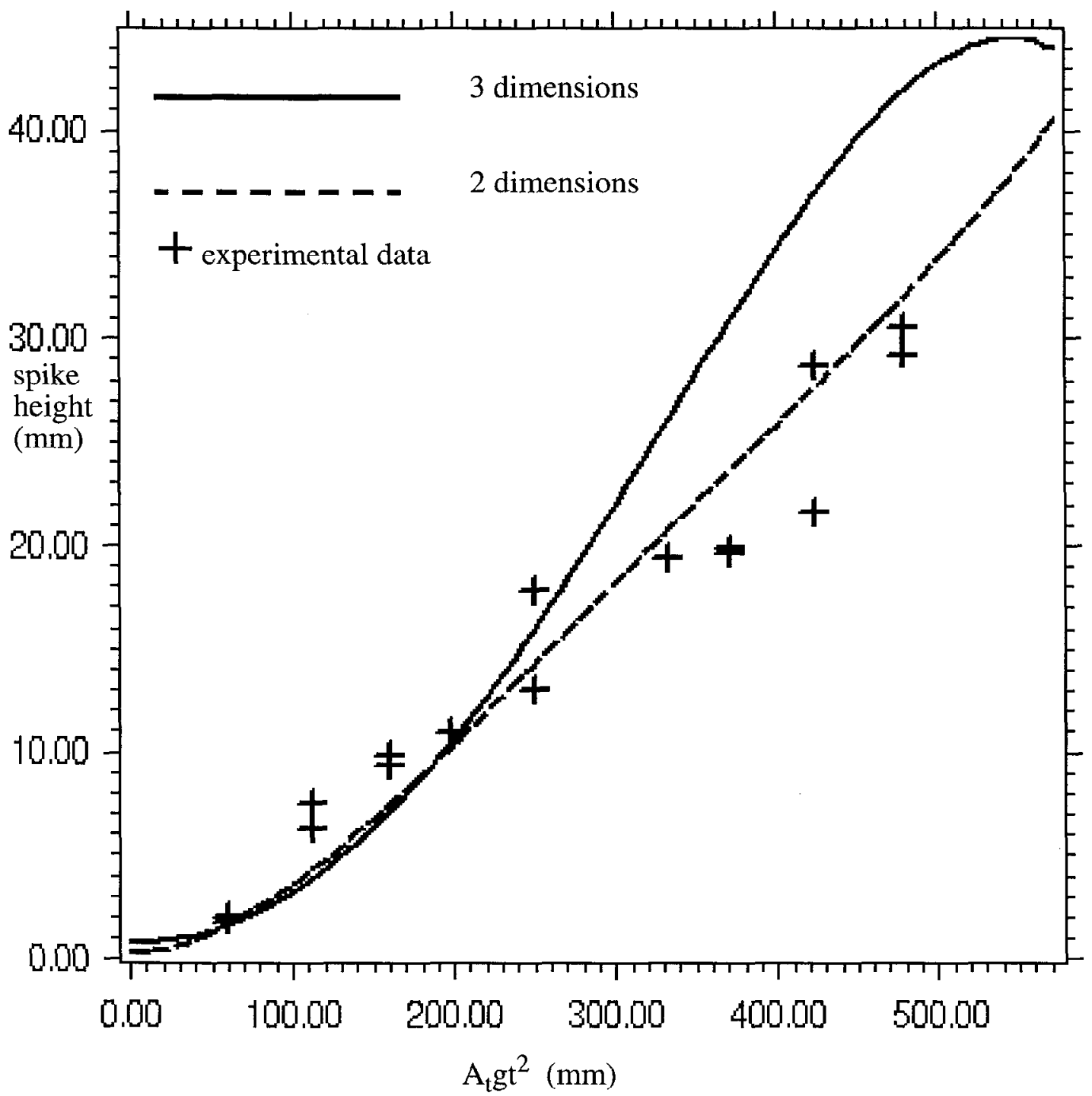

Fig. 39: Spike growth

The bubble growth rate in 2 dimensions was $\alpha_{2}=0.050$ while in 3 dimensions was $\alpha_{2}=0.075$. The 2 dimensional case was much closer to experimental and simulation values. This is because the initial magnitude of the perturbation was tuned to the 2 dimensional case. The choice of initial conditions is a source of inaccuracy in these simulations, and will be discussed further in the next section. 


\subsection{The effect of LES in 2 and 3 dimensions}

The accuracy of low resolution simulations can often be improved with the use of LES. LES can also be used in the creation of more realistic initial conditions. LES uses subgrid scale modeling to approximate unresolved energy. The Cloutman model [12] creates an additional conserved parameter, the subgrid scale kinetic $k$ (see Section 10.0 on page 42). An eddy viscosity is created from $k$, which turns an inviscid simulation into a solution to the full Navier-Stokes equations. The presence of eddy viscosity usually reduces the instability growth (see Section 12.0 on page 52 ) but in this instance in 2 dimensions growth slightly increased. 


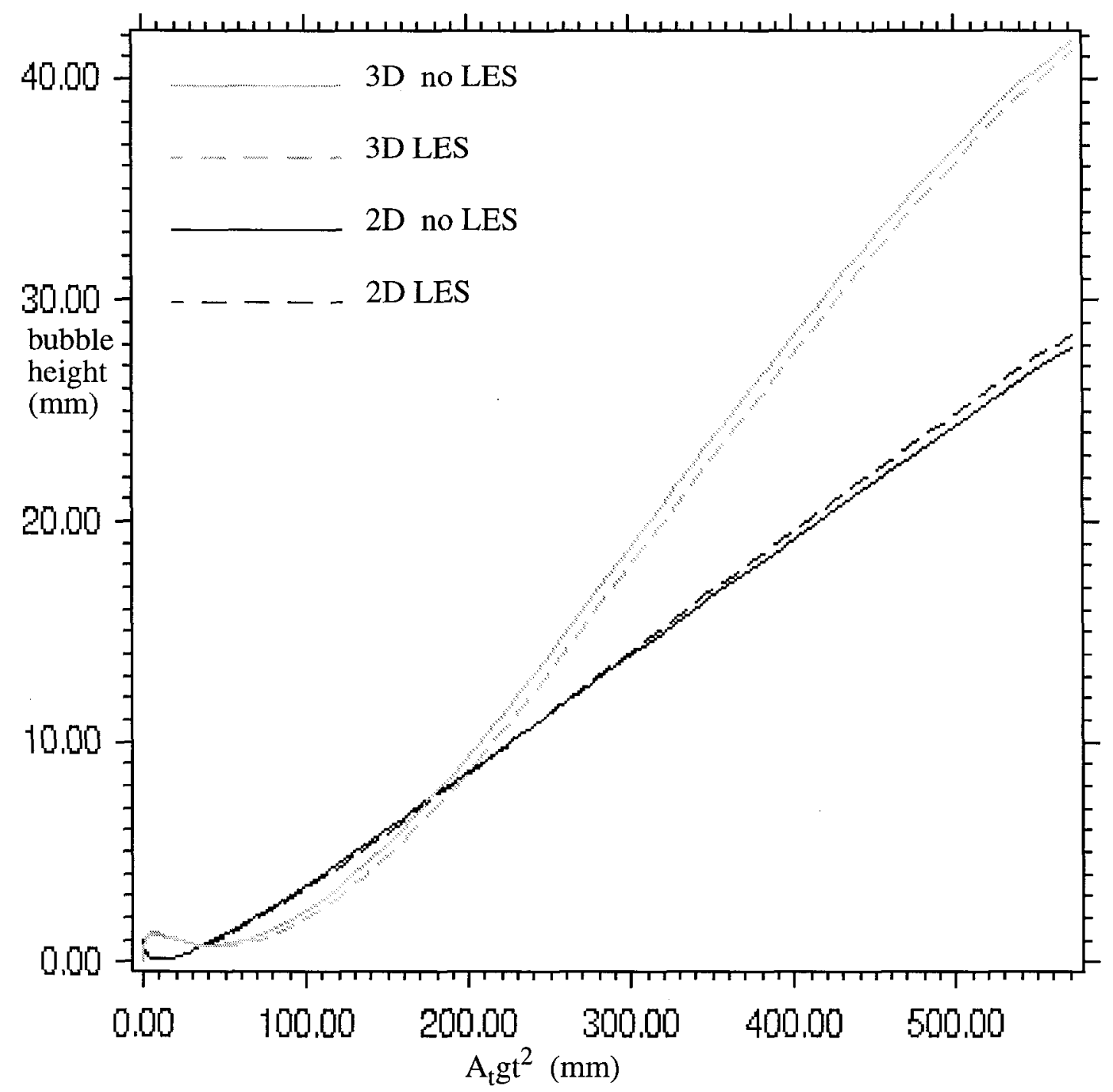

Fig. 40: Eddy viscosity and instability growth

One of the difficulties in simulating Linear Electric Motor experiments is in simulating the exact initial conditions of the experiment. It was impossible to recreate the exact conditions of the experiment with limited resolution, since the smallest perturbation modes were lost. The simulations, and perhaps the experiments as well, depended heavily on initial conditions. The simulations are largely in the nonlinear stage of Rayleigh-Taylor growth, and memory of the initial conditions is not lost until the turbulent 
stage. The magnitude of the initial perturbation had a great effect on the growth rate, making results questionable. It is important to create a perturbation whose effect is not so dependent on an arbitrary constant.

The LES model with stochastic backscatter (see Section 10.2 on page 47) should be a better approximation to the initial conditions than an imposed interface perturbation. P. Amala successfully used backscatter with LES to simulate a random Rayleigh-Taylor perturbation in [2]. The backscatter model creates random fluctuations near the interface which trigger Rayleigh-Taylor growth. In the current implementation of backscatter, the magnitude of the instability was based on the subgrid scale kinetic energy, which was coupled with the other equations of motion. However, in this case the perturbation grew too slowly. A perturbation in other fields besides the velocity might help.

Backscatter caused additional dissipation of subgrid scale kinetic energy. The reduction of subgrid scale energy therefore reduced the magnitude of the velocity perturbation. Increasing the amount of backscatter only increased growth to a limited extent. 


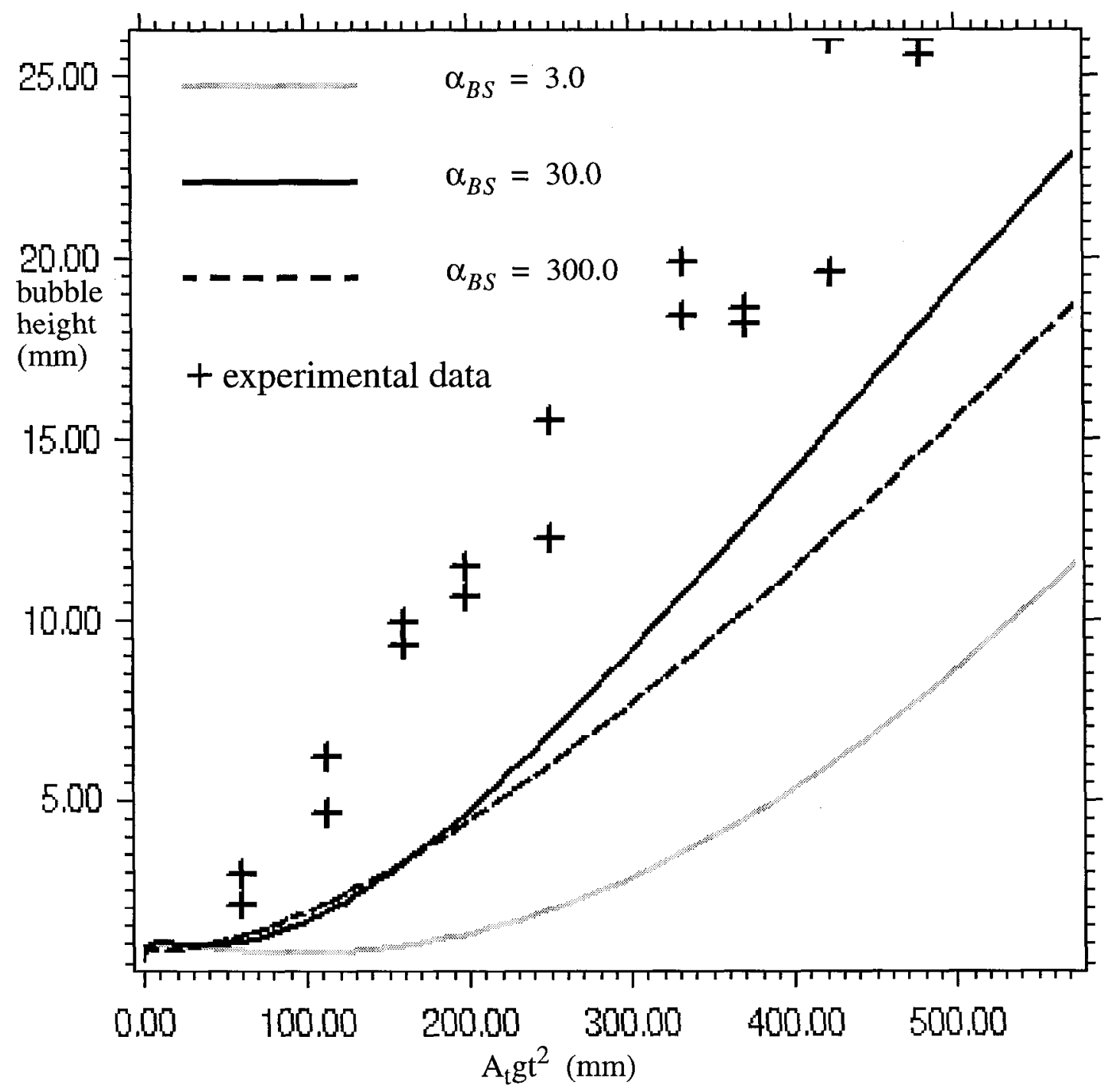

Fig. 41: Increasing backscatter in 2 dimensions

Backscatter caused more growth in 2 dimensions than 3, so dissipation effects are probably greater in 3 dimensions than 2 . 


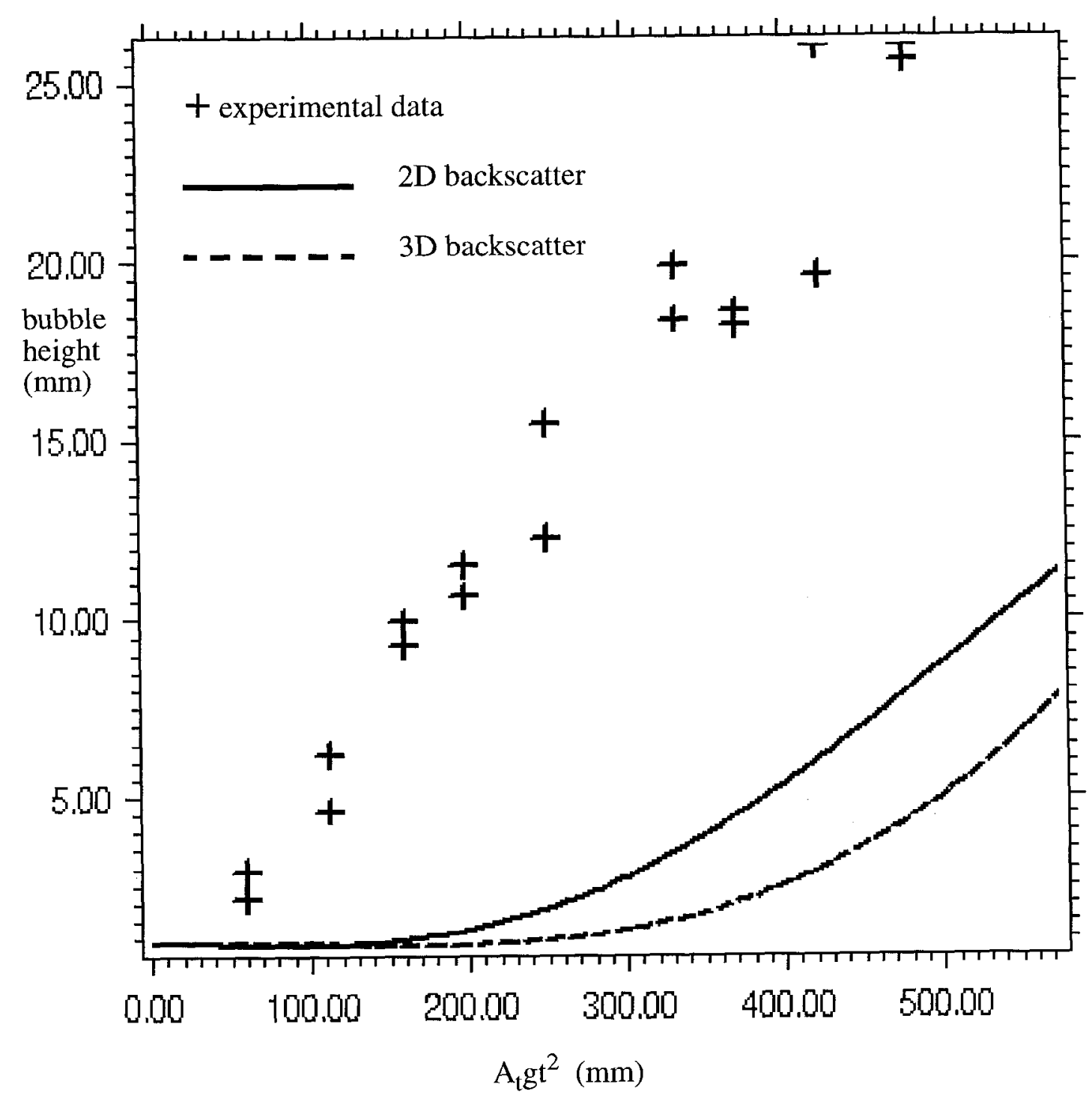

Fig. 42: Instability growth of backscatter without an initial perturbation imposed

Increasing resolution increased the growth of a perturbation caused by backscatter.

The velocity of each node is perturbed by a random quantity. Doubling the number of nodes increases the number of possible perturbation modes that can be created as a result of the random velocity. 


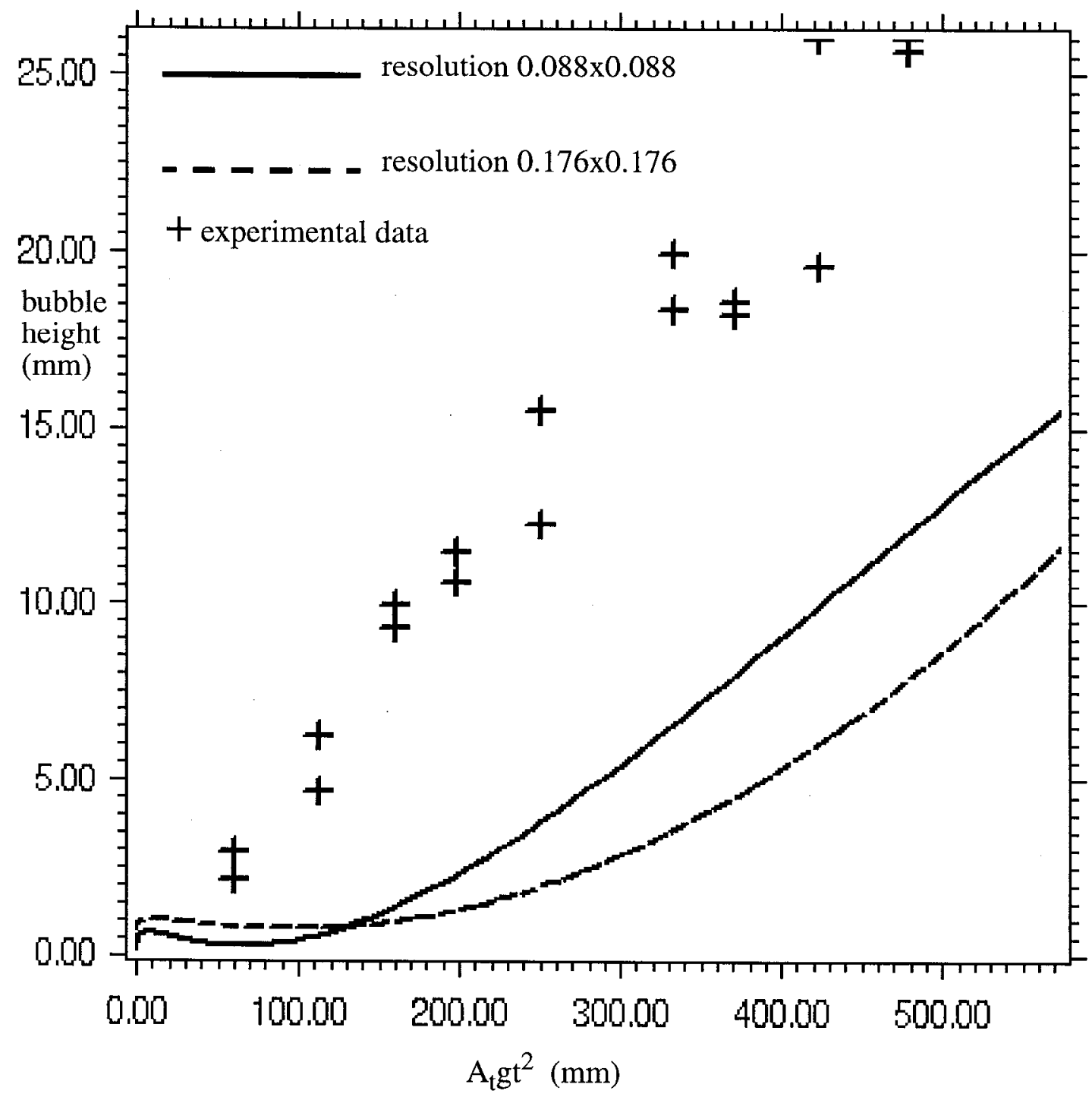

Fig. 43: Backscatter and resolution in 2 dimensions without an initial perturbation imposed

\subsection{Atwood number dependence}

The effect of Atwood number on Rayleigh-Taylor bubble and spike growth was studied by Dimonte [16] for density ratios between 1.3 and 50 . In this study, density ratios from 2.0 to 10.0 were simulated. As the density ratio was increased, the spikes became narrower and narrower until they were hard to resolve, so higher density ratios were not 
simulated. As the density ratio increased, so did the growth of the bubbles and spikes. In order to prevent the instability from hitting the top and bottom of the container in the experiments, the acceleration was reduced for higher density ratios. In these simulations, the domain was increased to $22.0 \mathrm{~cm}$ to fit all density ratios at the same acceleration. Scaling by $g t^{2}$, compressibility reduction, and a multimode interface were used as described in Section 16.1. The Atwood number study was in 2 dimensions.

Table 9: Growth Rates

\begin{tabular}{|l|l|l|l|}
\hline density ratio & $\begin{array}{c}\text { Atwood \# } \\
A_{t}\end{array}$ & $\alpha_{2}$ bubble & $\alpha_{1}$ spike \\
\hline \hline 2 & 0.333333 & 0.04521593 & 0.06222622 \\
\hline 3 & 0.5 & 0.04222986 & 0.07390172 \\
\hline 4 & 0.6 & 0.04056284 & 0.08045763 \\
\hline 5 & 0.666667 & 0.03968104 & 0.08461168 \\
\hline 6 & 0.714286 & 0.03846328 & 0.08127361 \\
\hline 7 & 0.75 & 0.03527236 & 0.08056597 \\
\hline 8 & 0.777778 & 0.03455228 & 0.08227720 \\
\hline 9 & 0.8 & 0.03093695 & 0.08685334 \\
\hline 10 & 0.818182 & 0.02684966 & 0.08910156 \\
\hline
\end{tabular}




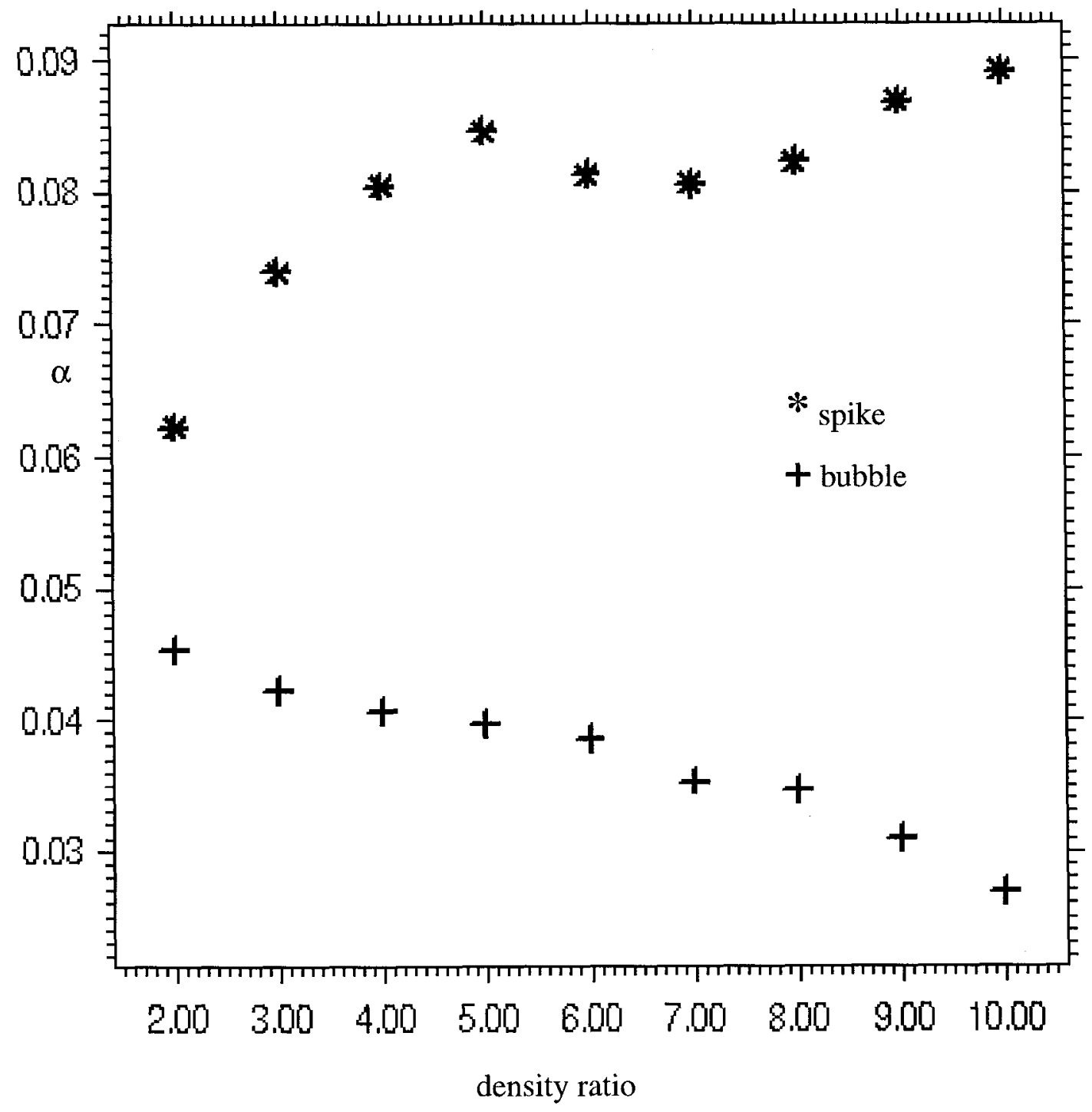

Fig. 44: Instability growth and density ratio

Instability growth was plotted against $A_{t} g t^{2}$ and the slope found through a linear fit for each density ratio. A downward trend in bubble growth rate is noticeable as density ratio increases. There was a much smaller downward trend in experimental measurements. Values for $\alpha_{2}$ were lower than experimental values as well. The spike growth rate increased with density ratio as expected, but at a slower rate than observed experi- 
mentally. Experimental results show $\alpha_{1}=0.052 R^{0.32 \pm 0.05}$ while simulation results show $\alpha_{1}=0.059 R^{0.18}$.

\subsection{Simulation observations}

Simulation of Rayleigh-Taylor instability experiments with the Linear Electric Motor show the adaptability of the methods employed in recreating experimental conditions. However, it was difficult to make a realistic approximation of the initial conditions. LES shows promise for this type of simulation, in creating a more realistic instability than the imposed perturbation used in this study. However the current implementation of backscatter had too small an effect.

Atwood number dependence differs from experiment, giving lower values for the growth rates of both bubble and spike. But the greatest difference occurred in the power law for density ratio in the spike. The simulation value was over $40 \%$ lower than the experimental value.

\subsection{Simulation of laser driven Rayleigh-Taylor instability experiments}

The previous section examined simulations of Rayleigh-Taylor instability experiments that were similar to the simplified test problems of the previous chapters. This section looks at simulations of experiments that use the ALE method to greater advantage. Mesh motion is increased, illustrating the importance of ALE in simulations of experiments. The usefulness of LES in approximating unresolved instability modes is also shown. 
Rayleigh-Taylor instability is particularly important to inertial confinement fusion (ICF) experiments. In indirect drive ICF, laser light shines on the interior of a gold covered cylindrical hohlraum. The laser radiation is reflected as an x-ray bath surrounding the target containing deuterium-tritium fuel. The fuel is surrounded by an ablator shell of brominated plastic.

The outside layer of the shell is vaporized by the $\mathrm{x}$-ray bath, and ablative acceleration occurs. Due to conservation of momentum, the outward acceleration of the vaporized (less dense) shell causes the non-vaporized (more dense) inner part of the shell to experience an inward acceleration. The acceleration of less dense into more dense shell material is the first case of possible Rayleigh-Taylor instability in the process. The lower density fuel also decelerates the inner surface of the higher density capsule material as it is compressed. This is the second case of possible Rayleigh-Taylor instability in the process. If Rayleigh-Taylor instability grows to such an extent that it causes the fuel and shell material to mix, the energy yield will be reduced.

Experiments by Budil et al. [4][5] address the growth of Rayleigh-Taylor instability in conditions relevant to ICF experiments. In [5], Budil et al. examined the growth of two Rayleigh-Taylor unstable modes that were initially too short in wavelength to be resolved experimentally. The growth of a larger beat mode produced by mode coupling was measured instead. The unstable interface was placed away from the stabilizing effect of the ablative acceleration to produce the most growth at short wavelengths.

Two layers of material were attached over a hole in the hohlraum with a sinusoidal interface perturbation imbedded between them. Lower density brominated plastic similar to the ICF ablator shell material was backed with higher density titanium. The exper- 
imental package was ablatively accelerated, and a Rayleigh-Taylor unstable condition $\nabla p \bullet \nabla \rho<0$ developed at the interface. The lower density brominated plastic was accelerated into the higher density titanium. The actual growth of the bubbles and spikes could not be viewed directly from the side of the target. Instead, an x-ray backlighter beam was used to radiograph the target, and the resulting data was analyzed to determine the amplitude of the constituent modes of the instability.

\subsection{Beat modes}

In [5], both initial interface modes (4 and $5 \mu \mathrm{m}$ wavelengths at $0.3 \mu \mathrm{m}$ amplitude) were smaller than the experimental resolution of about $7 \mu \mathrm{m}$. However, mode coupling produced a beat mode whose wavelength $(20 \mu \mathrm{m})$ was large enough to be resolved. This phenomenon can be observed in simulations as well.

A two mode instability at the interface of a Rayleigh-Taylor instability simulation can cause the growth of a larger wavelength beat mode in a simulation where neither mode is well resolved initially. The following simulation was done with two ideal gasses of different densities superimposed in a uniform acceleration. The two modes had wavelengths $\lambda_{1}=0.4$ and $\lambda_{2}=0.5 \mathrm{~cm}$, and amplitudes 0.01 . The grid resolution was $0.2 \times 0.2 \mathrm{~cm}$, so neither mode was well resolved initially. The instability grew with a clearly dominant mode of wavelength 2.0 , which is one of the beat modes for the sum of the two modes, as can be seen in Fig. 45 .

$$
\lambda_{\text {beat }}=\frac{\lambda_{1} \lambda_{2}}{\lambda_{2}-\lambda_{1}}=2.0
$$




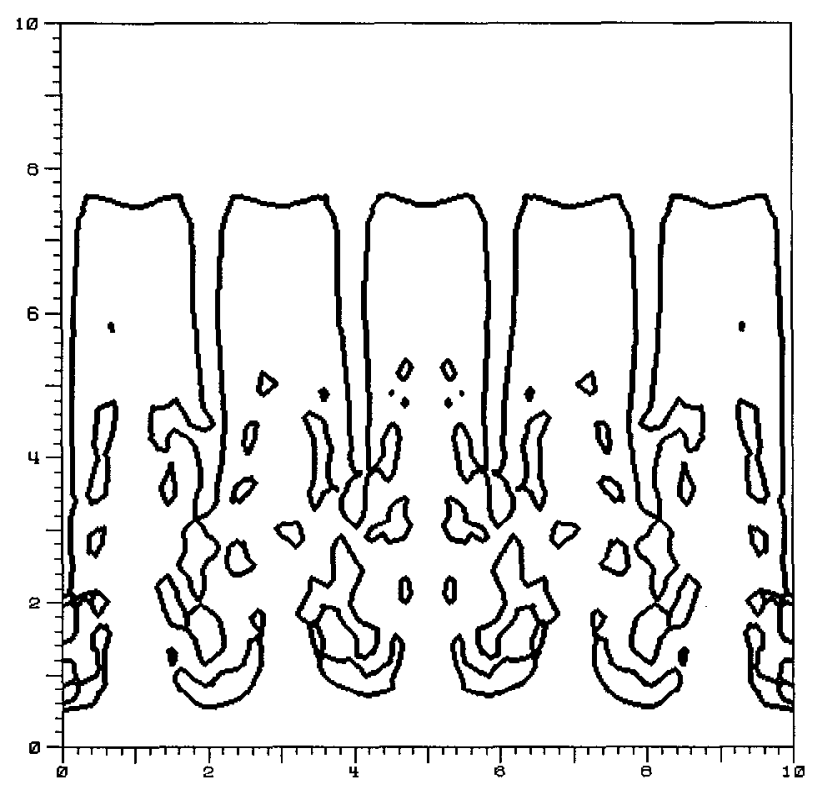

Fig. 45: The growth of a beat mode from a two mode interface at $0.2 \times 0.2$ resolution

The beat mode did not start growing immediately. According to Rayleigh-Taylor instability theory [59], interface instability modes grow independently for a short linear phase in their growth. After the linear phase, individual modes begin to couple as they grow further into the nonlinear phase. It is in the nonlinear phase that beat modes can appear. The nonlinear phase starts after the amplitude of the initial modes reaches about a tenth of their wavelength. However, linear theory alone does not account for the long delay before the growth of the beat mode in this simulation. The beat mode took 10 microseconds to begin to grow at all, which is more than enough time for the initial modes to grow past the linear phase if they were resolved.

Had the initial interface been well resolved, the two modes would have grown and interfered to produce many modes. The beat mode was not visible in this case. Symmetry was preserved, while many small scale features were produced by mode coupling. 


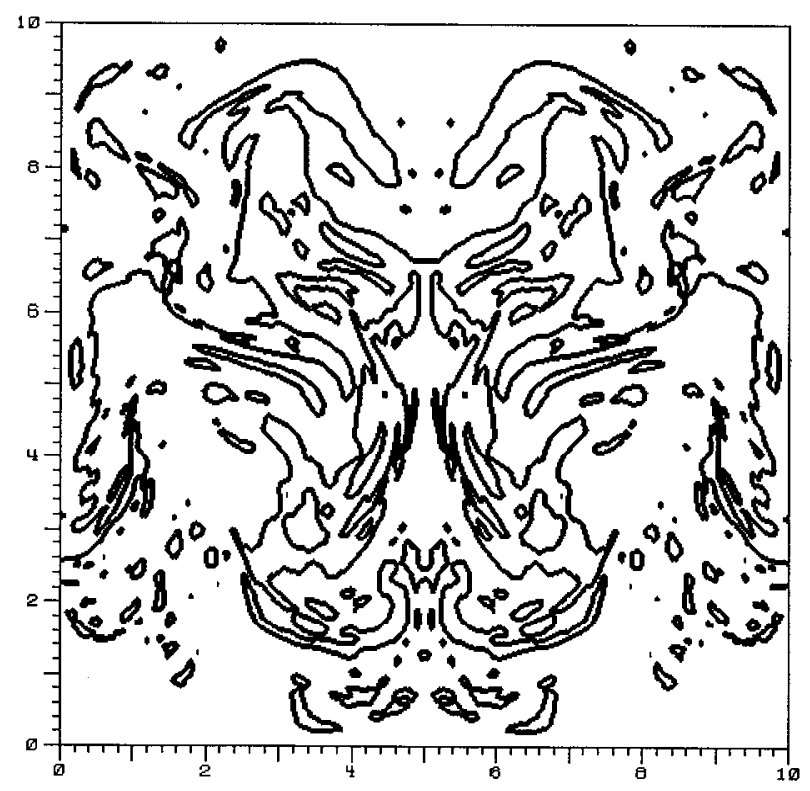

Fig. 46: Growth of two mode interface at $0.1 \times 0.1$ resolution

The addition of a subgrid scale model with stochastic backscatter (see Section 12.1 on page 55) enhanced the growth of the bubble. There are many ways to implement stochastic backscatter. In this study a random perturbation was added to the velocity field in the calculation of the strain rate tensor. The constant $\alpha_{B S}$ was set to 3.0. The random perturbation represents a sampling of an infinite number of unresolved modes. The magnitude of the perturbation was related to $\sqrt{k}$, where $k$ is the subgrid scale kinetic energy. Stochastic backscatter increased overall instability growth. However, symmetry was broken. Few small scale features were preserved, due to low resolution, but the overall growth of the instability was closer to that of the more highly resolved simulation in Fig. 46 . 


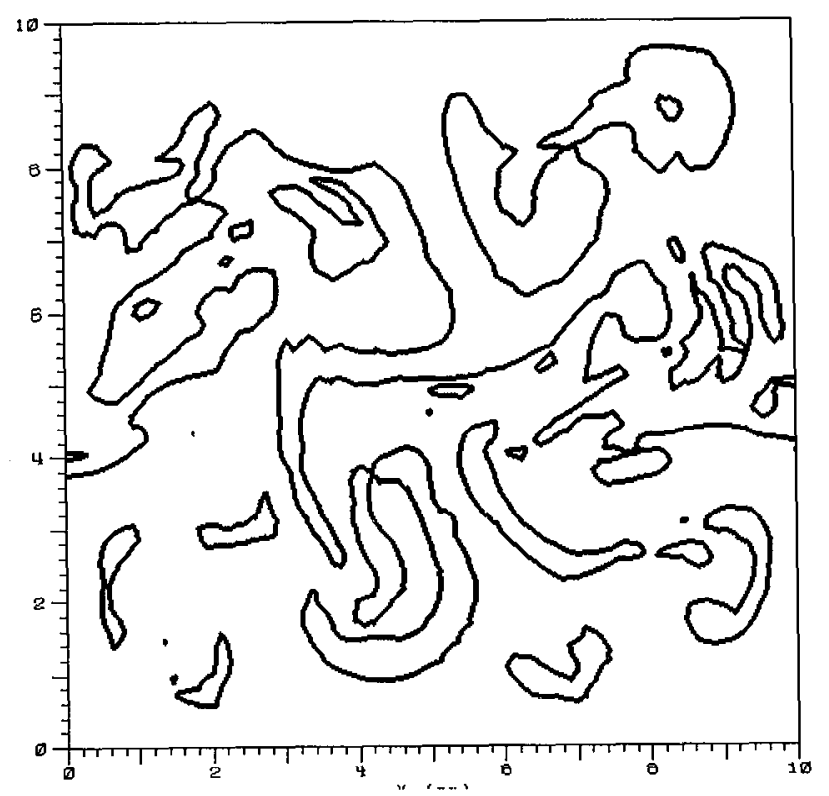

Fig. 47: Growth of two mode interface with backscatter at $0.2 \times 0.2$ resolution

The effect of backscatter on bubble growth is shown in Fig. 48 . The bubble height was measured from $1 \%$ to $99 \%$ by mass fraction, to track the edge of the instability as closely as possible. The step appearance of the growth is due to the grid resolution. 


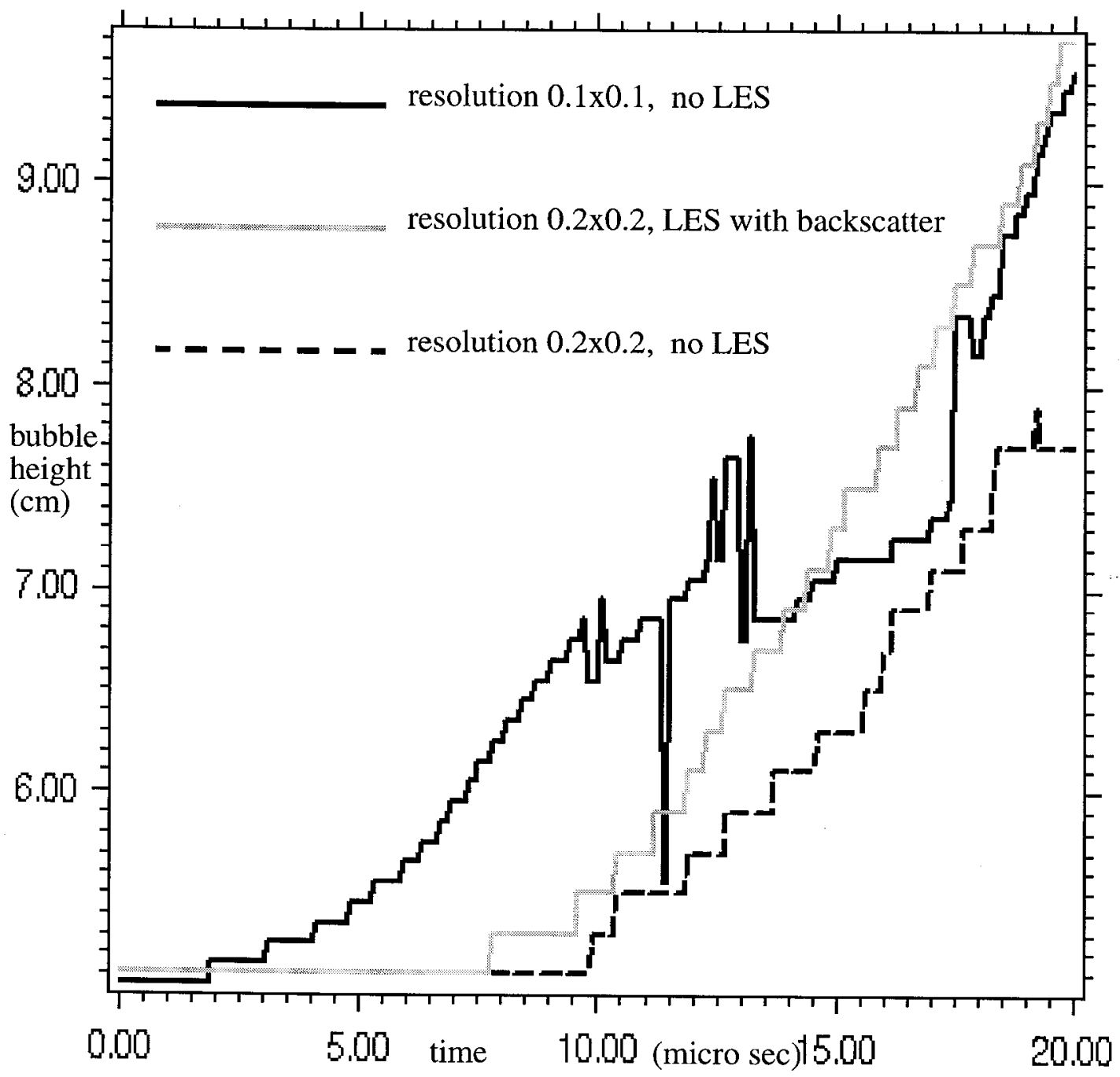

Fig. 48: Backscatter and instability growth

\subsection{Simulation details}

A more realistic simulation of the experiment was carried out without LES, using more realistic equations of state [70] than the ideal gas law. A two mode simulation was created with a horizontal resolution of $7 \mu m$ and initial perturbation modes of $4 \mu m$ and $5 \mu m$ in wavelength. The grid zones were initially clustered in the $55 \mu \mathrm{m}$ target (40 $\mu m$ brominated plastic backed by $15 \mu m$ Titanium), and a $450 \mu m$ layer of vacuum was 
also simulated below the target. In the vertical direction there were 221 zones in the target material and 100 zones in the vacuum. This was necessary since the target expanded vertically and filled the entire domain, and the lower boundary of the target developed ripples.

As can be seen in Fig. 50 , the ALE method allowed the mesh to spread with the target. In an Eulerian simulation, higher resolution would be required throughout the domain to follow the moving target. This would slow the simulation considerably. The resolution of the mesh within the target reduced over time, but the clustering of zones in the target made the resolution highest where it was most needed through the simulation. That is, ALE made better use a limited number of zones. 


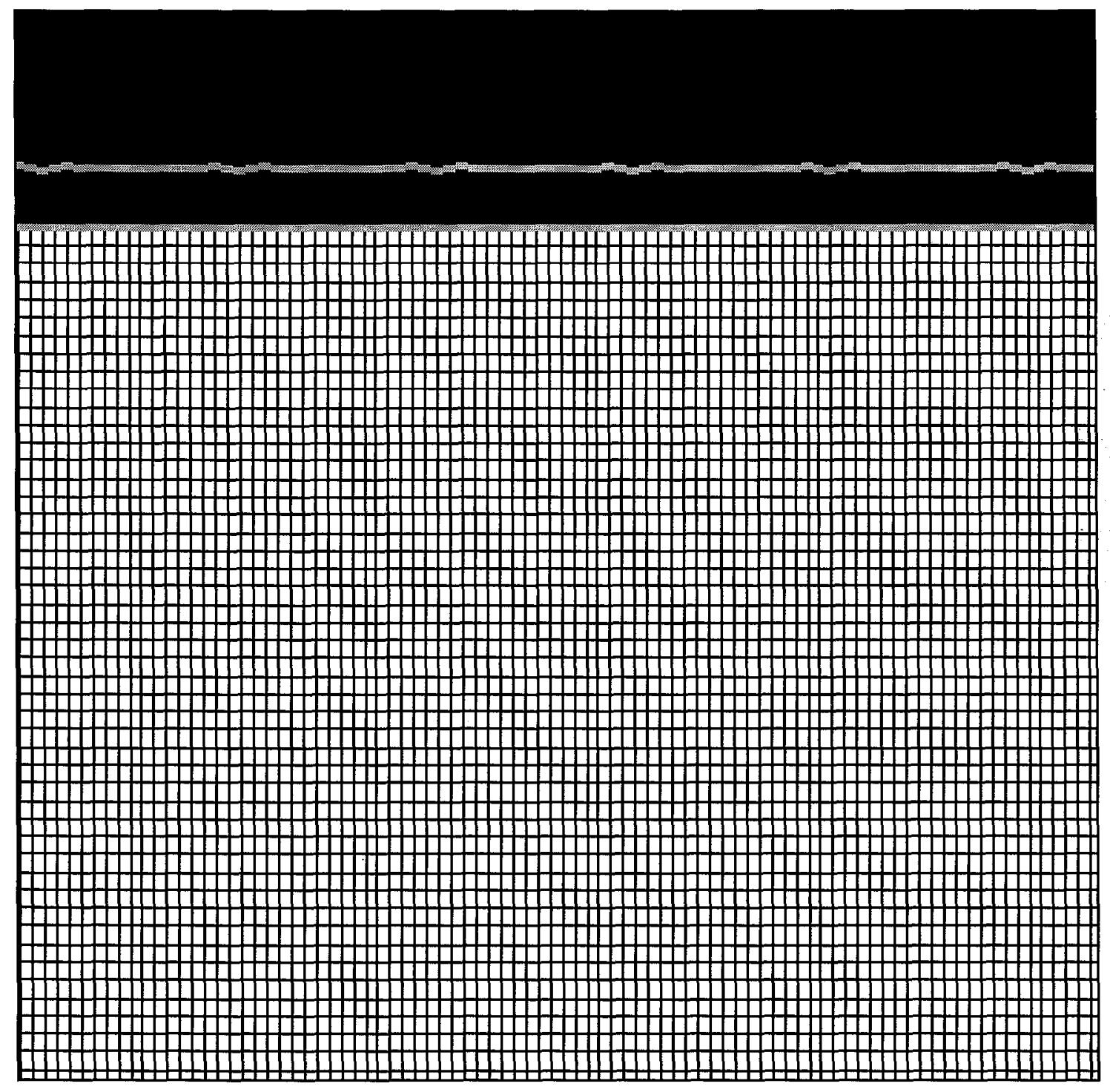

Fig. 49: Initial mesh 


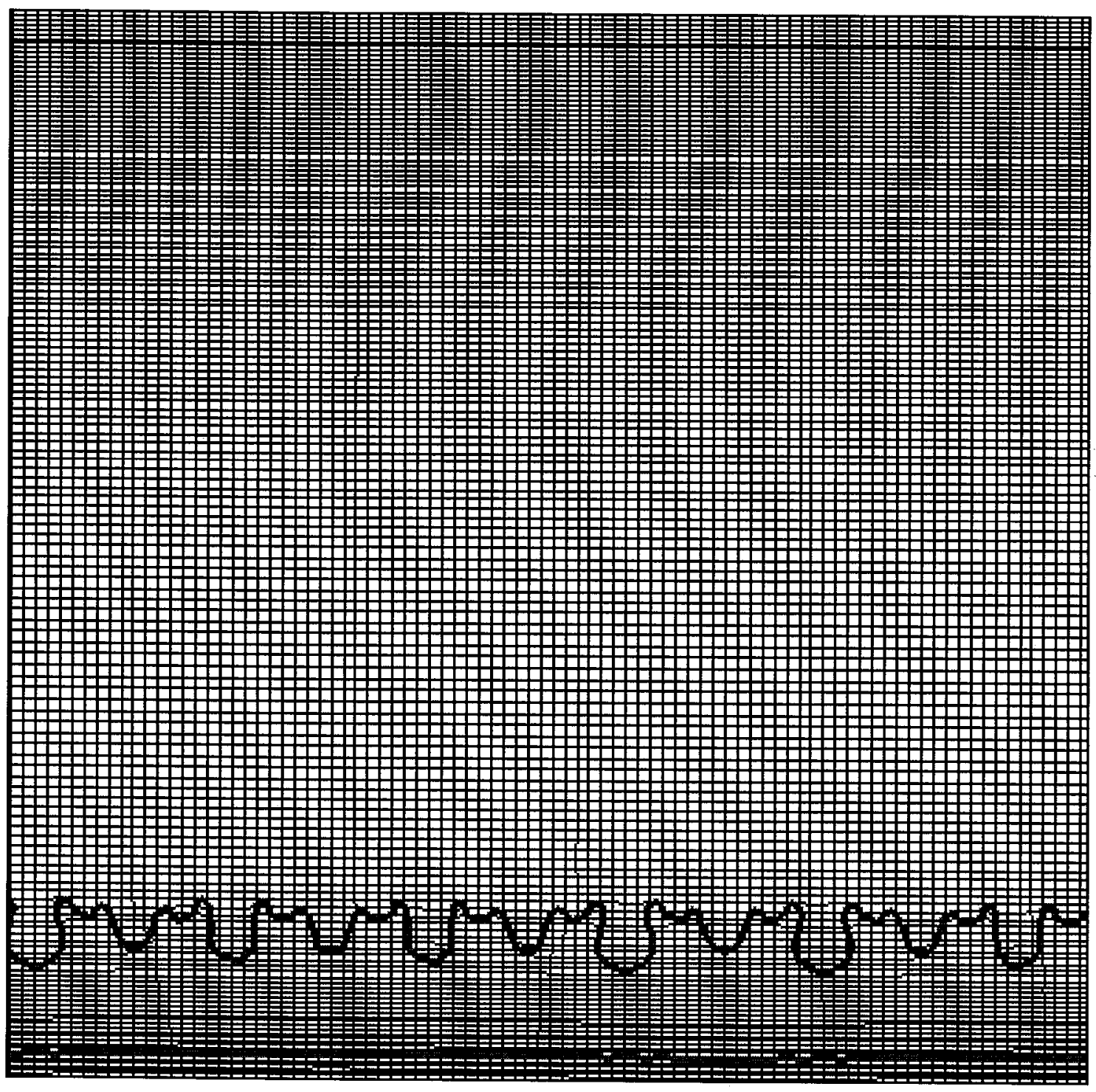

Fig. 50: Final mesh

The resulting interface clearly shows the presence of a mode that is larger than either of the constituent modes. This should be the beat mode at $20 \mu \mathrm{m}$. Analysis shows this mode to be about $33 \mu \mathrm{m}$, somewhat larger than the expected beat mode. 


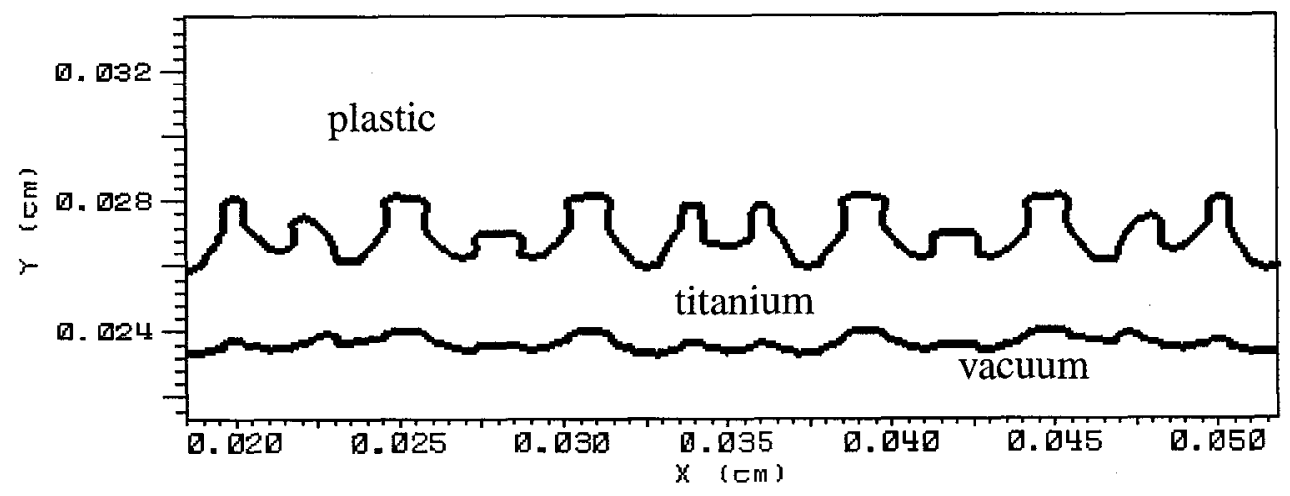

Fig. 51: Growth of two mode instability

\subsection{Conclusions}

The simulations of experiments in this chapter indicate that LES with ALE may be very useful in future applications. Both methods improve accuracy at lower resolutions, and this will be important until computers are large enough to run simulations that resolve all relevant scales. ALE follows the changing shape of the target, making lower resolution simulations more powerful, while LES simulates small scale motion.

Stochastic backscatter replaces specific unresolved interface modes with a spectrum of many unresolved modes. The resulting interface does not have the same shape as the fully resolved simulation, but the growth rate is more accurate. Therefore, stochastic backscatter can serve as a subgrid scale model for unresolved interface modes, allowing low resolution simulations to be more accurate.

There is a great deal of room for future research in this area. In particular, the behavior of the LES has not been tested in the ALE simulation of the laser driven RayleighTaylor instability experiment. Since the mesh motion is so much greater in these simulations than in test problems used previously, this would be a good test of the behavior of 
LES with ALE. 


\section{Conclusions}

The application of large eddy simulation (LES) to Arbitrary Lagrangian-Eulerian (ALE) simulations is a relatively new area of study. This research addresses three questions:

1. How does ALE mesh motion effect the simulation of Rayleigh-Taylor instability?

2. How does LES work with ALE mesh motion?

3. How can ALE mesh motion and LES be used together to improve experimental simulations?

The results of this research show that, without LES, ALE improves the ability of a mesh to capture a moving Rayleigh-Taylor interface. ALE more accurately captured the instability growth and energy balance than Eulerian simulations, as compared to higher resolution simulations.

LES worked well with ALE mesh motion in the cases studied. The performance of LES was similar with ALE and Eulerian mesh motion. Subgrid scale kinetic energy can be used as an indicator to the ALE mesh as to where zones are needed most. This response of the mesh to simulation conditions is impossible with fixed meshes.

Experimental simulations showed areas where LES can improve a simulation in conjunction with ALE. Stochastic backscatter in particular models the unresolved small scale instability modes at resolvable scales. Simulations of laser driven instability show the usefulness of ALE. The mesh zones were clustered in the target area, which spread in the course of the simulation to many times its original size. The zones also spread to follow the target, eliminating the need for high resolution throughout the simulation.

In conclusion, ALE improves simulation of Rayleigh-Taylor instability, and can be 
used in conjunction with LES to simulate unresolved flow features. The combination of ALE and LES should be important in future experimental simulations.

\subsection{Future research}

The behavior of LES in different ALE simulations could use more attention. This study concentrates the analysis of ALE and LES on simple simulations where ALE does not cause a great deal of mesh motion. Looking at the behavior of LES in simulations of laser driven Rayleigh-Taylor instabilities would show how they behave in the presence of greater mesh motion.

Stochastic backscatter is another area where improvements would be useful. Perturbing more fields than the velocity may increase the effect of the backscatter model. This model might then be more useful in creating the instability in Linear Flectric Motor experimental simulations, and in simulating unresolved modes in laser driven experimental simulations. 


\section{Appendix A: Rayleigh-Taylor Instability}

Rayleigh taylor instability occurs at a fluid interface if the pressure and density gradients are in opposite directions. That is, $\nabla p \cdot \nabla \rho<0$. If the interface between the two fluids is perfectly flat, the fluids remain in equilibrium with the pressure gradient balancing gravity. But infinitesimal perturbations in the interface grow exponentially. The heavy fluid moves into the light fluid, forming "spikes", and the light fluid moves into the heavy fluid, forming "bubbles". The shape of the interface can be Fourier analyzed to describe its component wavelengths.

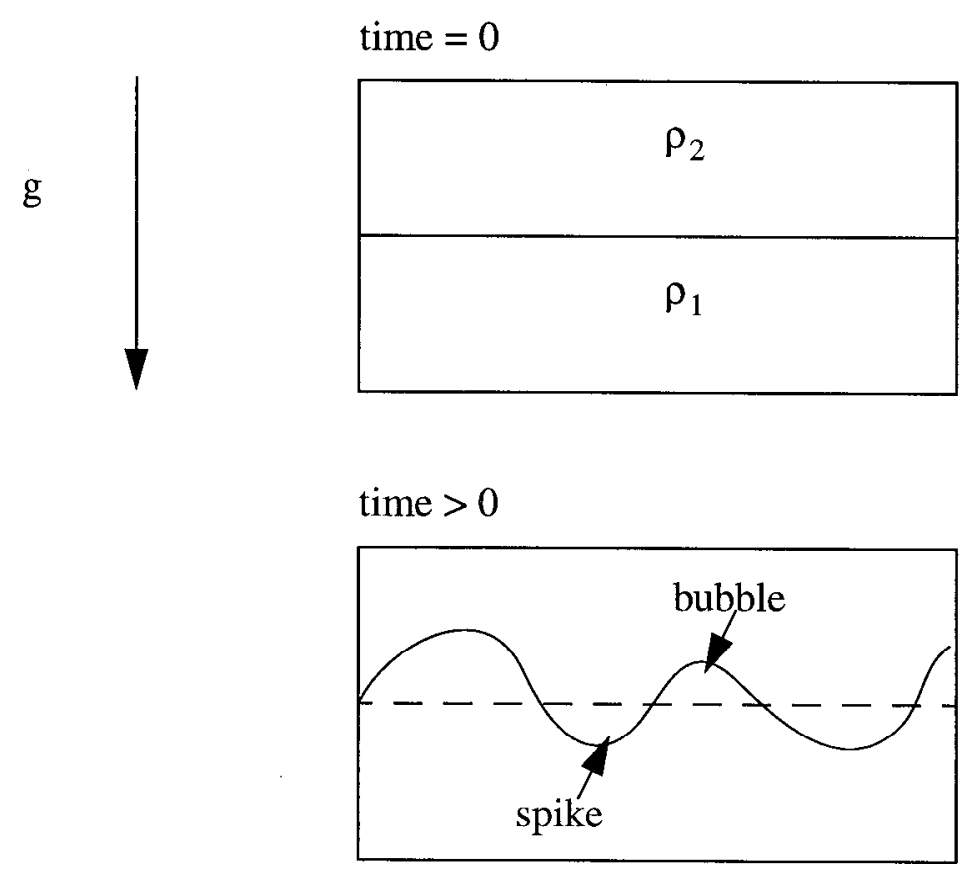

Fig. 1: Rayleigh-Taylor Instability

Rayleigh-Taylor instability has three stages [72]. First, in the linear stage, each perturbation wavelength grows independently according to linear theory. Next, in the nonlinear stage, the different wavelengths interact and slow individual growth. Finally, in 
the turbulent stage, all memory of initial conditions is lost.

\subsection{Linear stability analysis}

Chandrasekhar [9] describes the early stages of Rayleigh-Taylor instability by linear stability analysis for incompressible, inviscid fluids. Initially a stable equilibrium exists with the pressure gradient balancing gravity: $\nabla p=\rho g$. Gravity acts only in the $\mathrm{z}$ direction. A small perturbation in pressure and density is described by $p=p+\delta p$ and $\rho=\rho+\delta \rho$. Given $\frac{\partial}{\partial t} \delta \rho+u_{i} \frac{\partial \rho}{\partial x_{i}}=0$ the equations of motion become:

$$
\text { Conservation of mass: } \frac{\partial u_{i}}{\partial x_{i}}=0
$$

$$
\text { Conservation of momentum: } \quad \rho \cdot \frac{\partial u_{i}}{\partial t}=-\frac{\partial}{\partial x_{i}} \delta p-(g \delta \rho) \hat{z}
$$

Assuming a solution of the form $\exp \left(i k_{x} x+i k_{y} y+n t\right)$ for $\delta p$ and $\delta \rho$ the stability equation is

$$
\frac{d}{d z}\left(\rho \frac{d w}{d z}\right)-\rho k^{2} w=-\frac{k^{2}}{n^{2}} g \frac{d \rho}{d z} w
$$

where $w$ is the velocity in the $\mathrm{z}$ direction.

For two uniform fluids separated initially by a flat, horizontal boundary at $\mathrm{z}=0$, the stability equation becomes

$$
\frac{d^{2} w}{d x^{2}}-k^{2} w=0
$$


where $k^{2}=k_{x}^{2}+k_{y}^{2}=(2 \pi / \lambda)^{2}$. The boundary conditions are given by

$$
\lim _{\varepsilon \rightarrow 0}\left[\left.\left(\rho \frac{d w}{d z}\right)\right|_{0+\varepsilon}-\left.\left(\rho \frac{d w}{d z}\right)\right|_{0-\varepsilon}\right]=\frac{-k^{2}}{n^{2}}\left[g\left(\rho_{2}-\rho_{1}\right)-k^{2} T\right] w_{0}
$$

where $w$ vanishes at $\pm \infty$ and is continuous and equal to $w_{0}$ at $z=0 . T$ is surface tension. Therefore,

$$
n^{2}=g k\left[\frac{\rho_{2}-\rho_{1}}{\rho_{2}+\rho_{1}}-\frac{k^{2} T}{g\left(\rho_{2}+\rho_{1}\right)}\right]
$$

The fluids are Rayleigh-Taylor unstable if $\rho_{2}<\rho_{1}$ and $0<k<\sqrt{\frac{g}{T}\left(\rho_{2}-\rho_{1}\right)}$. Surface tension $T$ damps larger $\mathrm{k}$.

\subsection{Nonlinear growth}

Linear theory predicts an exponential growth rate for perturbations in a RayleighTaylor unstable fluid. However, in actual instabilities, linear theory only applies for a short time. In situations where the unstable interface is random, many unstable modes are present. The most unstable mode grows first, followed by other modes. Initially all modes grow independently according to linear theory, but they rapidly begin to interfere with each other and the linear solution no longer applies.

In single mode perturbations, the mode grows according to linear theory until it reaches about a tenth of its wavelength [52]. After this, nonlinear growth continues as additional unstable modes are produced. It has been found that single mode RayleighTaylor instabilities approach a constant velocity, while multimode or random instabili- 
ties approach a constant acceleration as they grow [1][38].

For a random interface [72], the most unstable wavelength grows first. Once the amplitude of the most unstable wavelength has reached approximately half its wavelength, the nonlinear stage begins. Finally, when the dominant wavelength's amplitude has reached about ten times the most unstable wavelength, a similarity solution can be applied.

\subsection{Similarity solution}

In the turbulent stage of Rayleigh-Taylor instability, the initial conditions are forgotten and surface tension is neglected. Youngs [72] assumes a similarity solution with $n t=$ const so that

$$
h=\alpha A_{t} g t^{2}
$$

The instability width is $h$, although this is usually applied to bubble growth rather than the entire instability growth. The Atwood number is defined as $A_{t}=\frac{\rho_{2}-\rho_{1}}{\rho_{2}+\rho_{1}}$. The constant $\alpha$ has been measured for many experiments and simulations, with results ranging between 0.03 and 0.07 . The effect of compressibility and viscosity is to reduce the growth rate [59]. 


\section{Appendix B: Solving the Equations of Motion}

\subsection{Euler equations}

Since molecular viscosity is neglected, the inviscid equations of motion are solved when subgrid scale models are not used. In the Lagrangian step of this Arbitrary Lagrangian-Eulerian (ALE) method, the grid moves with the fluid. The equations of motion are solved for one time step. This is done predictor corrector for second order accuracy. The inviscid equations of motion in Lagrangian coordinates are:

$$
\begin{gathered}
\text { Conservation of Mass: } \frac{1}{\rho} \frac{d \rho}{d t}=-\nabla \bullet u \\
\text { Conservation of Momentum: } \rho \frac{d u}{d t}=-\nabla p+\rho g \\
\text { Conservation of Internal Energy: } \rho \frac{d e}{d t}=-p \nabla \bullet u
\end{gathered}
$$

Definitions:

$\frac{d}{d t}=\frac{\partial}{\partial t}+u \cdot \nabla$ Lagrangian derivative

$t$ time

$\rho$ density

$u$ velocity vector

$p$ pressure (for example, by the ideal gas law $p=(\gamma-1) \rho e$ where $\gamma=\frac{5}{3}$ )

$g$ gravitational acceleration

$e$ internal energy per unit mass 


\subsection{Conservation of mass}

In solving the equations of motion, EQ 8 and EQ 10 are coupled together. First, EQ 8 is integrated from $t$ to $t^{\prime}$ over the appropriate time step, either $\frac{\Delta t}{2}$ for the predictor step or $\Delta t$ for the corrector step.

$$
\begin{gathered}
\int_{\rho}^{\rho^{\prime}} \frac{d \rho}{\rho}=-\int_{t}^{t^{\prime}} \nabla \bullet u d t \\
\rho^{\prime}=\rho \exp \left(-\int_{t}^{t^{\prime}} \nabla \bullet u d t\right)
\end{gathered}
$$

Since the mesh follows the fluid, the mass in a zone remains constant throughout the Lagrangian step so $\rho^{\prime}=\rho \frac{V}{V^{\prime}}$. Therefore,

$$
\begin{gathered}
\frac{V}{V^{\prime}}=\exp \left(-\int_{t}^{t^{\prime}} \nabla \bullet u d t\right) \text { or } \\
\frac{V^{\prime}}{V}=\exp \left(\int_{t}^{t^{\prime}} \nabla \bullet u d t\right)
\end{gathered}
$$

Expanding the exponential in a Taylor series to first order and solving for its argument,

$$
\int_{t}^{t^{\prime}} \nabla \bullet u d t=\frac{V^{\prime}-V}{V}
$$

This approximation is used to solve the internal energy equation (see Section 4.3).

\subsection{Conservation of momentum}

To advance the velocity equation in time from $t$ to $t^{\prime}, \mathrm{EQ} 9$ is discretized for the 
appropriate time step, either $\frac{\Delta t}{2}$ for the predictor step or $\Delta t$ for the corrector step.

$$
u^{\prime}=u+\Delta t a
$$

The acceleration $a$ is derived by a finite volume scheme developed by Wilkins for the HEMP code [69]. The momentum equation is integrated over the volume of a mesh zone, $V$, which has surface $S$. Using Green's theorem, the volume integral of the pressure gradient becomes a surface integral.

$$
\begin{aligned}
& \text { x momentum equation: } \int_{V} \rho \frac{d u_{x}}{d t} d V=-\int_{V} \frac{\partial p}{\partial x} d V=-\int_{S} p d y \\
& \text { y momentum equation: } \int_{V} \rho \frac{d u_{y}}{d t} d V=-\int_{V} \frac{\partial p}{\partial y} d V=\int_{S} p d x
\end{aligned}
$$

This process is repeated for each mesh zone. The density and velocily derivatives are assumed to be constant throughout the zone volume, so they come out of the integral. The nodes and zones in question are numbered as follows:

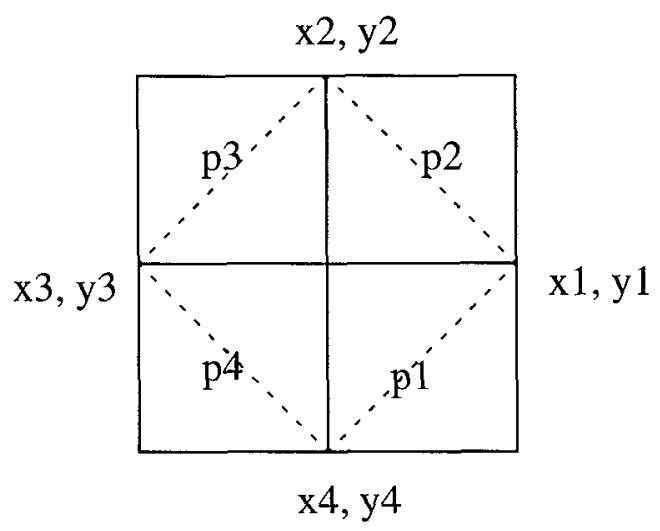

Using the midpoint rule, the acceleration is approximated in terms of the $\mathrm{x}$ or $\mathrm{y}$ values at the four nodes and the pressure values at the four zone centers. The resulting accelera- 
tion is node centered. Gravitational acceleration is added to the appropriate acceleration terms. In this case, gravity is in the y direction.

$$
\begin{gathered}
a_{x}=-\frac{1}{\rho A}[p 2(y 2-y 1)+p 3(y 3-y 2)+p 4(y 4-y 3)+p 1(y 1-y 4)] \\
a_{y}=-\frac{1}{\rho A}[p 2(x 1-x 2)+p 3(x 2-x 3)+p 4(x 3-x 4)+p 1(x 4-x 1)]+g
\end{gathered}
$$

The units of the acceleration should be $\frac{\text { velocity }}{\text { time }}=\frac{\text { pressure } \times \text { area }}{\text { mass }}$. This formulation uses the form $\frac{\text { pressure } \times \text { length }}{\text { density } \times \text { area }}$ instead, which amounts to the same units.

\subsection{Conservation of internal energy}

The internal energy equation (EQ 10) is first integrated over the zonal volume $V$ :

$$
\int_{V} \rho \frac{d e}{d t} d V=m \frac{d e}{d t}=-\int_{V} p \nabla \bullet u d V
$$

Next, EQ 21 is integrated over time and the order if integration on the right is

exchanged. Pressure is evaluated at time $t^{n}$ for the predictor step and time $t^{n+\frac{1}{2}}$ for the corrector step.

$$
\int_{t}^{t^{\prime}} m \frac{d e}{d t} d t=-\int_{t}^{t^{\prime}}\left(\int_{V} p \nabla \bullet u d V\right) d t=-\int_{V}\left(p \int_{t}^{t^{\prime}} \nabla \bullet u d t\right) d V
$$

Using EQ 15, the right hand side is simplified. The midpoint rule is used, so pressure is evaluated at the zone centroid.

$$
m\left(e^{\prime}-e\right)=-\int_{V} p \frac{\left(V^{\prime}-V\right)}{V} d V=-p \frac{\left(V^{\prime}-V\right)}{V} V
$$


The final form for the change in internal energy becomes $P \Delta V$.

$$
e^{\prime}=e-p \frac{\left(V^{\prime}-V\right)}{\rho V}
$$

This can be reached a simpler way through the second law of thermodynamics:

$T d s=d e+p d V$ where $s$ is entropy. In the case where $d s=0, d e=-p d V$.

\subsection{Predictor step}

In the predictor step, time is advanced from $t^{n}$ to $t^{n+\frac{1}{2}}$ using a forward difference approximation in time, giving first order accuracy.

Advance velocity in time: $u^{n+\frac{1}{2}}=u^{n}+\frac{\Delta t}{2} \cdot a^{n}$

Move grid to follow the fluid: $x^{n+\frac{1}{2}}=x^{n}+\frac{\Delta t}{2} \cdot u^{n}$

$$
\text { Advance density in time: } \rho^{n+\frac{1}{2}}=\frac{\rho^{n} V^{n}}{V^{n+\frac{1}{2}}}
$$

Advance internal energy in time: $e^{n+\frac{1}{2}}=e^{n}-p^{n}\left(\frac{V^{n+\frac{1}{2}}-V^{n}}{\rho^{n} V^{n}}\right)$

\subsection{Corrector step}

The corrector step uses the predictor step to make a central difference approximation in time, at second order accuracy. Time is advanced from $t^{n}$ to $t^{n+1}$. Then, to keep the half time step values at second order accuracy, the first order values are replaced with averages of second order values. 
Advance velocity in time: $u^{n+1}=u^{n}+\Delta t a^{n+\frac{1}{2}}$

Average half time velocity to second order accuracy: $u^{n+\frac{1}{2}}=\frac{1}{2}\left(u^{n}+u^{n+1}\right)$

Move grid to follow the fluid: $x^{n+1}=x^{n}+\Lambda t u^{n+\frac{1}{2}}$

$$
\text { Advance density in time: } \rho^{n+1}=\frac{\rho^{n} V^{n}}{V^{n+1}}
$$

Advance internal energy in time: $e^{n+1}=e^{n}-p^{n+\frac{1}{2}}\left(\frac{V^{n+1}-V^{n}}{\rho^{n} V^{n}}\right)$

\subsection{Equations of motion for the Cloutman model}

For the Cloutman model [12], the entire viscous compressible equations of motion apply.

$$
\begin{gathered}
\text { Conservation of Mass: } \frac{1}{\rho} \frac{d \rho}{d t}=-\nabla \bullet u \\
\text { Conservation of Momentum: } \rho \frac{d u}{d t}=-\nabla p+\nabla \bullet\left(\mu \varepsilon_{i j}\right)+\rho g
\end{gathered}
$$

$$
\text { Conservation of Internal Energy: } \rho \frac{d e}{d t}=\nabla \bullet(\kappa \nabla T)-p \nabla \bullet u+\mu \varepsilon_{i j}: \nabla u
$$

Definitions:

$$
\frac{d}{d t}=\frac{\partial}{\partial t}+u \cdot \nabla \text { Lagrangian derivative }
$$

$t$ time

$\rho$ density

$u$ velocity vector 
$p$ pressure (for example, by the ideal gas law $p=(\gamma-1) \rho e$ where $\gamma=\frac{5}{3}$ )

$\varepsilon_{i j}=\frac{\partial u_{i}}{\partial x_{j}}+\frac{\partial u_{j}}{\partial x_{i}}-\frac{2}{3} \nabla \bullet u \delta_{i j}$ strain rate tensor

$\tau_{i j}=-\delta_{i j} p+\mu\left(\frac{\partial u_{i}}{\partial x_{j}}+\frac{\partial u_{j}}{\partial x_{i}}\right)+\delta_{i j} \lambda \nabla \bullet u$ stress tensor

$\mu, \lambda$ dynamic viscosity of the fluid $\lambda=-\frac{2}{3} \mu$ by Stoke's hypothesis

$v=\mu / \rho$ kinematic viscosity of the fluid

$g$ gravitational acceleration

$e$ internal energy per unit mass

$\kappa$ internal energy diffusivity

$T$ tempcrature: $T=e / c_{v}$ in this case

$c_{v}=\frac{\partial e}{\partial T}$ heat capacity at constant volume

These equations are filtered to separate the resolved scales from the subgrid scales. A

new variable, $k$, the subgrid scale kinetic energy, is created. After numerous approximations (see Section 10.0 on page 42), the equations of motion become:

$$
\text { Conservation of Mass: } \frac{d}{d t} \tilde{\rho}=-\tilde{\rho} \nabla \bullet \bar{u}
$$

Conservation of Momentum: $\tilde{\rho} \frac{d}{d t} \bar{u}=-\nabla\left(\tilde{p}+p_{t}\right)+\nabla \bullet\left(\left(\mu+\mu_{t}\right) \bar{\varepsilon}_{i j}\right)+\tilde{\rho} \bar{g}$ 


$$
\begin{gathered}
\text { Conservation of Internal Energy: } \\
\tilde{\rho} \frac{d}{d t} \bar{e}=\nabla \bullet(\kappa \nabla \bar{T})+\nabla \bullet\left(\kappa_{t} \nabla \bar{e}\right)-\tilde{p} \nabla \bullet \bar{u}+\mu \bar{\varepsilon}_{i j}: \nabla \bar{u}+S I N K-S O U R C E \\
\text { Conservation of Turbulent Kinetic Energy: } \\
\tilde{\rho} \frac{d k}{d t}=\nabla \bullet\left(\left(\mu+\mu_{t}\right) \nabla k\right)-p_{t} \nabla \bullet \bar{u}+\mu_{t} \bar{\varepsilon}_{i j}: \nabla \bar{u}-S I N K+S O U R C E \\
S I N K=D \tilde{\rho} \frac{\frac{k}{2}}{\lambda} \\
\text { SOURCE }=-B \mu_{t}\left(\frac{\nabla \tilde{\rho}}{\tilde{\rho}} \cdot \frac{\nabla \tilde{p}}{\tilde{\rho}}\right)_{i f<0} \\
\text { Turbulent Pressure: } p_{t}=\frac{2}{3} \tilde{\rho} k \\
\text { Turbulent Viscosity: } \mu_{t}=A \tilde{\rho} \lambda \sqrt{k} \\
\text { Turbulent Thermal Diffusivity: } \kappa_{t}=\frac{\mu_{t}}{P r_{t}}
\end{gathered}
$$

Definitions:

$$
\begin{aligned}
& \langle f\rangle=\tilde{f}=\text { filtered } f \\
& \bar{f}=\langle\rho f\rangle /\langle\rho\rangle
\end{aligned}
$$

Eddy viscosity is assumed to be much larger than molecular viscosity. Therefore, $\mu$,

$$
\kappa=\frac{\mu c_{p}}{P r} \text {, and } D=\frac{\mu}{S c} \text { are neglected. This is a source of error as the grid resolution }
$$

becomes smaller. As resolution comes closer to the Kolmogorov scale, the subgrid scale models will turn off. At this point the molecular viscosity should become significant, but in this case the simulation will become inviscid. It is assumed the resolution will not approach the Kolmogorov scale. It is also assumed that $\operatorname{Pr}_{t}=S c_{t}=0.7$, the values 
for an ideal gas.

\subsection{Solving the equations of motion}

The method for solving the equations of motion is much the same as for the Euler equations, with a few added steps. The strain rate tensor is created using a variation of the finite volume method described in Section 4.2. The velocities are node centered, so their derivatives become zone centered. The momentum equation becomes:

$$
\begin{gathered}
\rho \frac{d u_{x}}{d t}=-\frac{\partial p}{\partial x}+\frac{\partial \tau_{x x}}{\partial x}+\frac{\partial \tau_{x y}}{\partial y} \\
\rho \frac{d u_{y}}{d t}=-\frac{\partial p}{\partial y}+\frac{\partial \tau_{y x}}{\partial x}+\frac{\partial \tau_{y y}}{\partial y}+\rho g
\end{gathered}
$$

The derivatives of the pressure and stress tensor are calculated with the method described in Scction 4.2.

For the internal energy equation and the turbulent kinetic energy equation, the stress tensor is contracted with the velocity gradient. The stress tensor is symmetric so $\tau_{i j}=\tau_{j i}$. The stress tensor is also defined to be traceless so $\tau_{i i}=0$. That is, the stress tensor we are actually using is:

$$
\left[\begin{array}{ccc}
\tau_{x x} & \tau_{x y} & 0 \\
\tau_{y x} & \tau_{y y} & 0 \\
0 & 0 & -\left(\tau_{x x}+\tau_{y y}\right)
\end{array}\right]
$$

This allows the simplification of the contraction.

$$
\tau_{i j}: \nabla u=\tau_{i j} \frac{\partial u_{i}}{\partial x_{j}}=\tau_{i j} \frac{1}{2}\left[\left(\frac{\partial u_{i}}{\partial x_{j}}+\frac{\partial u_{j}}{\partial x_{i}}\right)+\left(\frac{\partial u_{i}}{\partial x_{j}}-\frac{\partial u_{j}}{\partial x_{i}}\right)\right]
$$


Since $\tau_{i j}$ is symmetric and $\left(\frac{\partial u_{i}}{\partial x_{j}}-\frac{\partial u_{j}}{\partial x_{i}}\right)$ is antisymmetric, their product is zero. The product of $\tau_{i j}$ and $\frac{2}{3} \nabla \bullet u \delta_{i j}$ is also zero, since $\tau_{i j}$ is traceless. Therefore,

$$
\tau_{i j}: \nabla u=\frac{1}{2} \tau_{i j}\left(\frac{\partial u_{i}}{\partial x_{j}}+\frac{\partial u_{j}}{\partial x_{i}}-\frac{2}{3} \nabla \bullet u \delta_{i j}\right)=\frac{1}{2} \tau_{i j} \varepsilon_{i j}
$$

Since the components of the strain rate tensor were already calculated in creating the stress tensor, this saves computational time.

The Cloutman model adds diffusion, source, and sink terms to the internal and turbulent kinetic energy equations. The internal energy equation (EQ 39) and the turbulent kinetic energy equation (EQ 40) have very similar structure, and are solved in a similar way. Each equation is solved in three parts: diffusion, work, and source/sink. The changes to the energy fields incurred by each of the three processes are added together to give the total change in energy. This occurs for both the predictor and corrector step in solving the Lagrangian equations of motion. Using turbulent kinetic energy as an example, the three parts to the solution are:

1. Diffusion: $\tilde{\rho} \frac{d k}{d t}=\nabla \bullet\left(\mu_{t} \nabla k\right)$

2. Work: $\tilde{\rho} \frac{d k}{d t}=-p_{t} \nabla \bullet \bar{u}+\mu_{t} \bar{\varepsilon}_{i j}: \nabla \bar{u}$

3. Source/Sink: $\tilde{\rho} \frac{d k}{d t}=-D \tilde{\rho} \frac{k^{\frac{3}{2}}}{\lambda}-B \mu_{t}\left(\frac{\nabla \tilde{\rho}}{\tilde{\rho}} \cdot \frac{\nabla \tilde{p}}{\tilde{\rho}}\right)_{i f<0}$ 


\subsubsection{Diffusion}

The diffusion terms in the internal and turbulent kinetic energy equations are solved by an explicit finite element approach using a staggered mesh. The turbulent kinetic energy equation is used as an example. First, EQ 48 is integrated over the zonal volume V

$$
\int_{V} \tilde{\rho} \frac{d k}{d t}=\int_{V} \nabla \bullet\left(\mu_{t} \nabla k\right)
$$

The time derivative of $k$ is constant throughout $V$ so it is removed from the integral. Gauss's Law is used to convert the right hand side to an integral over the surface of the zone $S$.

$$
\frac{d k}{d t} m=\int_{S} \mu_{t} \nabla k \cdot d S
$$

A finite difference approximation is used for the time derivative, and a finite element approach is used for the surface integral. This converts EQ 51 to a matrix equation which can be solved explicitly.

$$
k^{\prime}=k+\frac{\Delta t}{m} A k
$$

\subsubsection{Work}

The pressure and stress work terms in EQ 40 are solved by a finite volume method (see Section 4.2).

Initially, turbulent kinetic energy $k$ is zero throughout the domain. It can be created by the buoyancy source term $-B \mu_{t}\left(\frac{\nabla \tilde{\rho}}{\tilde{\rho}} \cdot \frac{\nabla \tilde{p}}{\tilde{\rho}}\right)_{i f<0}$. A fluid which is Rayleigh-Taylor 
unstable will create $k$ through buoyancy, but a Kelvin-Helmholtz unstable fluid will not.

A seed term is needed to create $k$ in zones where the buoyancy source term does not apply, but $k$ is created due to viscous stress. The seed term for $k$ is developed by explicitly integrating the pressure and stress terms in the $k$ equation:

$$
\tilde{\rho} \frac{d k}{d t}=-p_{t} \nabla \bullet \bar{u}+\mu_{t} \overline{\varepsilon_{i j}}: \nabla \bar{u}
$$

Using EQ 41 and EQ 42 and cancelling density:

$$
\frac{d k}{d t}=-\frac{2}{3} k \nabla \bullet \bar{u}+A \sqrt{k} \lambda \overline{\varepsilon_{i j}}: \nabla \bar{u}=-\alpha k+\beta \sqrt{k}
$$

EQ 53 is integrated over one half time step for the predictor step.

$$
\int_{k^{n}}^{k^{n+\frac{1}{2}}} \frac{d k}{-\alpha k+\beta \sqrt{k}}=\int_{t}^{t+\frac{\Delta t}{2}} d t
$$

Solving for $k^{n+\frac{1}{2}}$ :

$$
k^{n+\frac{1}{2}}=\left[\left(\sqrt{k^{n}}-\frac{\beta}{\alpha}\right) \exp \left(-\alpha \frac{\Delta t}{2}\right)+\frac{\beta}{\alpha}\right]^{2}
$$

$k^{n}$ is zero, and the exponential is expanded in a Taylor series to second order. Terms involving $\alpha$ cancel, so the resulting seed term depends only on the viscous stress term $\beta$.

$$
k^{n+\frac{1}{2}}=\left(\beta \frac{\Delta t}{2}\right)^{2}
$$


This seed term is used only in the predictor step to seed $k$ only in zones where it is zero.

This produces a non-zero turbulent pressure and turbulent stress tensor which may be used in the corrector step. It couples the $k$ equation directly to the momentum equation for turbulent production.

\subsubsection{Source and sink}

The terms describing loss and gain of turbulent kinetic energy $k$ are solved explicitly.

Using $\mu_{t}=A \tilde{\rho} \lambda \sqrt{k}$, EQ 50 becomes:

$$
\tilde{\rho} \frac{d k}{d t}=-D \tilde{\rho} \frac{k^{\frac{3}{2}}}{\lambda}-B A \tilde{\rho} \lambda \sqrt{k}\left(\frac{\nabla \tilde{\rho}}{\tilde{\rho}} \cdot \frac{\nabla \tilde{p}}{\tilde{\rho}}\right)_{i f<0}
$$

Cancelling density, EQ 54 can be written as $\frac{d k}{d t}=\alpha k^{\frac{1}{2}}-\beta k^{\frac{3}{2}}$ where $\alpha=-B A \lambda\left(\frac{\nabla \tilde{\rho}}{\tilde{\rho}} \cdot \frac{\nabla \tilde{p}}{\tilde{\rho}}\right)_{i f<0}$ represents the source and $\beta=\frac{D}{\lambda}$ represents the sink. An explicit solution is found by integrating from time $t$ to $t^{\prime}$ over a half time step $\frac{\Delta t}{2}$ for the predictor step or a full time step $\Delta t$ for the corrector step.

$$
\int_{k^{n} \alpha k^{\frac{1}{2}}-\beta k^{\frac{3}{2}}}^{k^{\prime}}=\int_{t}^{t^{\prime}} d t
$$

Solving for $k^{\prime}$ : 


$$
k^{\prime}=\left(\frac{\sqrt{\frac{\alpha}{\beta}} \tanh \left(\frac{\Delta t}{2} \sqrt{\alpha \beta}\right)+\sqrt{k}}{1+\sqrt{\frac{\beta}{\alpha}} \tanh \left(\frac{\Delta t}{2} \sqrt{\alpha \beta}\right) \sqrt{k}}\right)^{2}
$$

In the course of a simulation, the source term may at any time decrease to zero, regardless of the value of $k$. But the sink term will always be present wherever there is turbulent kinetic energy. As it stands, the source and sink are linked so that if either is zero, both are zero. In the case where $\alpha$ is zero (no source) the hyperbolic tangent in EQ 55 is expanded in a Taylor series, decoupling the source and sink.

$$
k^{\prime}=\left(\frac{\alpha \frac{\Delta t}{2}+\sqrt{k}}{1+\beta \frac{\Delta t}{2} \sqrt{k}}\right)^{2}
$$

The change in energy due to the source and sink is added to the $k$ equation (EQ 40) and subtracted from the internal energy equation (EQ 39) to maintain energy conservation.

\subsection{Stochastic backscatter}

Subgrid scale turbulent fluctuations add a random component to large scale motion. Subgrid scale models without stochastic backscatter miss this component. A large scale flow which is initially symmetric remains symmetric throughout the simulation. This is not typical of real flows, so a stochastic backscatter term can be added to the model to simulate the effect of unresolved fluctuations on resolved scales.

A random perturbation is added to the velocity field in calculation of the strain rate tensor only. This preserves Galilean invariance. In the strain rate tensor 
$\bar{\varepsilon}_{i j}=\frac{\partial \bar{u}_{i}}{\partial x_{j}}+\frac{\partial \bar{u}_{i}}{\partial x_{i}}-\frac{2}{3} \frac{\partial \bar{u}_{k}}{\partial x_{k}} \delta_{i j}$ in the conservation equations for momentum (EQ 38), internal encrgy (EQ 39), and turbulent kinetic energy (EQ 40), a random velocity $u_{i}^{R}$ is added to the filtered velocity $\bar{u}_{i}=\bar{u}_{i}+u_{i}^{R}$.

The magnitude of $u_{i}^{R}$ is related to the subgrid scale kinetic energy $k$. We choose the cumulative distribution function of the random component of the velocity field to be:

$$
P\left(a, u_{i}^{R}\right)=\frac{\int_{0}^{u_{i}^{R}} x^{2} e^{-a x} d x}{\int_{0}^{\infty} x^{2} e^{-a x} d x}
$$

This distribution was chosen since it can be solved by integration by parts with the following results:

$$
P\left(a, u_{i}^{R}\right)=1-e^{-a u_{i}^{R}}\left(1+a u^{R}+\frac{1}{2}\left(\alpha u^{R}\right)^{2}\right)
$$

The mean value of $x$ is $\bar{x}=\frac{3}{a}$, so we chose $\bar{x}=\alpha_{B S} \sqrt{k}$ to determine $a=\frac{\alpha_{B S}}{3} \sqrt{k}$.

The constant $\alpha_{B S}$ is set by the user. To find the random velocity fluctuation, a random number $R$ between 0 and 1 is found (similar to Numerical Recipes [52] function ran2 p. 282) which replaces $P\left(a, u_{i}{ }^{R}\right.$. Solving for $u_{i}^{R}$,

$$
-a u_{i}^{R}+\ln \left(1+a u_{i}^{R}+\frac{1}{2}\left(a u_{i}^{R}\right)^{2}\right)=\ln (1-R)
$$

The natural log on the left is expanded in a Taylor Series to third order. Terms above cubic were discarded. The resulting magnitude of the velocity fluctuation is: 


$$
u_{i}^{R}=\frac{\alpha_{B S} \sqrt{k}}{3}(-6 \ln (1-R))^{1 / 3}
$$

More random numbers are chosen to give the direction of the velocity fluctuation over a unit sphere. In 2 dimensions, one random angle is required, and in 3 dimensions, two are required.

\subsection{Predictor step}

In the predictor step, time is advanced from $t^{n}$ to $t^{n+\frac{1}{2}}$ using a forward difference approximation in time, giving first order accuracy. The variables now represent filtered quantities, except for $k$ which is subgrid scale.

Advance velocity in time: $u^{n+\frac{1}{2}}=u^{n}+\frac{\Delta t}{2} \cdot a^{n}$

Move grid to follow the fluid: $x^{n+\frac{1}{2}}=x^{n}+\frac{\Delta t}{2} \cdot u^{n}$

$$
\text { Advance density in time: } \rho^{n+\frac{1}{2}}=\rho^{n} \cdot \frac{V^{n}}{V^{n+\frac{1}{2}}}
$$

Advance internal energy in time: $e^{n+\frac{1}{2}}=e^{n}+\Delta e_{1}+\Delta e_{2}+\Delta e_{3}$

Diffusion: $\Delta e_{1}=\frac{\Delta t}{2 m} A^{n} e^{n}$

$$
\operatorname{PdV} \text { work: } \Delta e_{2}=-p^{n}\left(\frac{V^{n+\frac{1}{2}}-V^{n}}{\rho^{n} V^{n}}\right)
$$




$$
\text { Source/sink: } \Delta e_{3}=\left(\frac{\sqrt{\frac{\alpha}{\beta}} \tanh \left(\frac{\Delta t}{4} \sqrt{\alpha \beta}\right)+\sqrt{k^{n}}}{1+\sqrt{\frac{\beta}{\alpha}} \tanh \left(\frac{\Delta t}{4} \sqrt{\alpha \beta}\right) \sqrt{k^{n}}}\right)^{2}-k^{n}
$$

Advance turbulent kinetic energy in time: $k^{n+\frac{1}{2}}=k^{n}+\Delta k_{1}+\Delta k_{2}+\Delta k_{3}+\Delta k_{4}$

$$
\begin{gathered}
\text { Diffusion: } \Delta k_{1}=\frac{\Delta t}{2 m} A^{n} k^{n} \\
\text { PdV work: } \Delta k_{2}=-p_{t}^{n}\left(\frac{V^{n+\frac{1}{2}}-V^{n}}{\rho^{n} V^{n}}\right) \\
\text { Stress work: } \Delta k_{3}=\frac{\Delta t}{2 m}\left(\frac{1}{2} \mu_{t} \varepsilon_{i j}^{n} \varepsilon_{i j}^{n}\right) V^{n} \\
\text { Source/sink: } \Delta k_{4}=\left(\frac{\sqrt{\frac{\alpha}{\beta}} \tanh \left(\frac{\Delta t}{4} \sqrt{\alpha \beta}\right)+\sqrt{k^{n}}}{1+\sqrt{\frac{\beta}{\alpha}} \tanh \left(\frac{\Delta t}{4} \sqrt{\alpha \beta}\right) \sqrt{k^{n}}}\right)^{2}-k^{n}
\end{gathered}
$$

\subsection{Corrector step}

The corrector step uses the predictor step to make a central difference approximation in time, at second order accuracy. Time is advanced from $t^{n}$ to $t^{n+1}$. Then, to keep the half time step values at second order accuracy, the first order values are replaced with averages of second order values.

$$
\text { Advance velocity in time: } u^{n+1}=u^{n}+\Delta t \cdot a^{n+\frac{1}{2}}
$$

Average half time velocity to second order accuracy: $u^{n+\frac{1}{2}}=\frac{1}{2} \cdot\left(u^{n}+u^{n+1}\right)$ 
Move grid to follow the fluid: $x^{n+1}=x^{n}+\Delta t \cdot u^{n+\frac{1}{2}}$

Average half time grid to second order accuracy: $x^{n+\frac{1}{2}}=\frac{1}{2} \cdot\left(x^{n}+x^{n+1}\right) \quad$ (EQ 74)

$$
\text { Advance density in time: } \rho^{n+1}=\rho^{n} \cdot \frac{V^{n}}{V^{n+1}}
$$

Advance internal energy in time: $e^{n+1}=e^{n}+\Delta e_{1}+\Delta e_{2}+\Delta e_{3}$

$$
\text { Diffusion: } \Delta e_{1}=\frac{\Delta t}{m} A^{n+\frac{1}{2}} e^{n+\frac{1}{2}}
$$

$$
\text { PdV work: } \Delta e_{2}=-p^{n+\frac{1}{2}}\left(\frac{V^{n+1}-V^{n}}{\rho^{n} V^{n}}\right)
$$

$$
\text { Source/sink: } \Delta e_{3}=\left(\frac{\sqrt{\frac{\alpha}{\beta}} \tanh \left(\frac{\Delta t}{2} \sqrt{\alpha \beta}\right)+\sqrt{k^{n}}}{1+\sqrt{\frac{\beta}{\alpha}} \tanh \left(\frac{\Delta t}{2} \sqrt{\alpha \beta}\right) \sqrt{k^{n}}}\right)^{2}-k^{n}
$$

Advance turbulent kinetic energy in time: $k^{n+1}=k^{n}+\Delta k_{1}+\Delta k_{2}+\Delta k_{3}+\Delta k_{4}$

$$
\begin{gathered}
\text { Diffusion: } \Delta k_{1}=\frac{\Delta t}{m} A^{n+\frac{1}{2}} k^{n+\frac{1}{2}} \\
\text { PdV work: } \Delta k_{2}=-p_{t}^{n+\frac{1}{2}}\left(\frac{V^{n+1}-V^{n}}{\rho^{n} V^{n}}\right) \\
\text { Stress work: } \Delta k_{3}=\frac{\Delta t}{m}\left(\frac{1}{2} \mu_{t} \varepsilon_{i j}^{\left.n+\frac{1}{2} \varepsilon_{i j}^{n+\frac{1}{2}}\right) V^{n+\frac{1}{2}}}\right.
\end{gathered}
$$




$$
\text { Source/sink: } \Delta k_{4}=\left(\frac{\sqrt{\frac{\alpha}{\beta}} \tanh \left(\frac{\Delta t}{2} \sqrt{\alpha \beta}\right)+\sqrt{k^{n}}}{1+\sqrt{\frac{\beta}{\alpha}} \tanh \left(\frac{\Delta t}{2} \sqrt{\alpha \beta}\right) \sqrt{k}^{n}}\right)^{2}-k^{n}
$$




\section{Appendix C: Grid Relaxation}

\subsection{Motivation for grid relaxation}

The Arbitrary Lagrangian-Eulerian (ALE) method in this study has two steps. In the Lagrangian step, the equations of motion are solved in order to advance the fluid from time $t$ to time $t+\Delta t$. The mesh moves with the fluid, but parts of it may need to be moved back, to reduce the distortion of the grid. This is the Eulerian or remap step, which allows the simulation to continue to follow a complicated flow in the next time step.

In the Eulerian step, a new grid is chosen, using information from the old grid. Then material is moved between grid zones in the new mesh, to respond to the new position of grid points in the fluid. The fluid does not advance in time during the Eulerian step, and the equations of motion are not solved. However, approximations are made when material is moved from one mesh zone to another. Therefore, density, velocity and energy may change slightly during the Eulerian step.

\subsection{Grid Generation}

The easiest grid to use mathematically is a uniform, orthogonal grid in a rectangular region. But fluid dynamics problems don't always conform to such a mesh. The Lagrangian step of an ALE code requires the mesh to follow the flow, which means the distribution of zones within the domain is constantly changing, and boundaries between fluids are moving.

An effective way to represent this is to transform a uniform, orthogonal mesh onto the 
physical space of the problem [17][34][63]. This adds a great deal of extra math to an already complicated problem. But it has several advantages. Suppose a grid (let's take an orthogonal one for example) were chosen to fit a curved boundary:

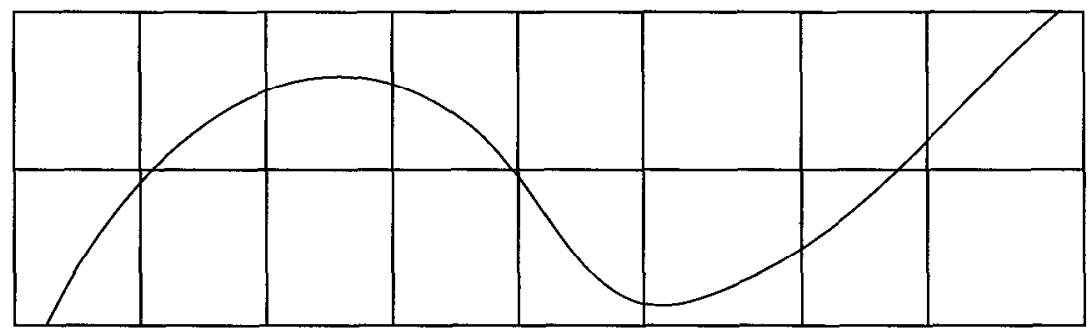

There are several problems with this:

1. It is hard to define the boundary, since it does not fall on coordinate lines.

2. The grid spacing next to the boundary is not even with the rest of the grid spacing. This causes centered difference approximations to lose accuracy.

3. Grid points are not evenly spaced on the boundary, making some areas poorly defined. To put extra grid points on boundary, extra grid points are also created inside, which makes the program slower.

These three problems can be solved by transforming a uniform, orthogonal grid in "logical space" onto the "physical space" of the actual fluid flow.

Logical Space $\quad(\xi, \eta)$

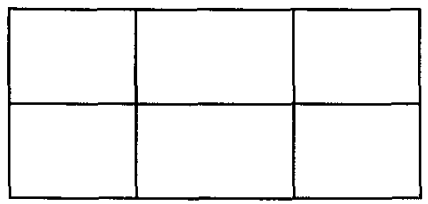

Physical Space (x,y)

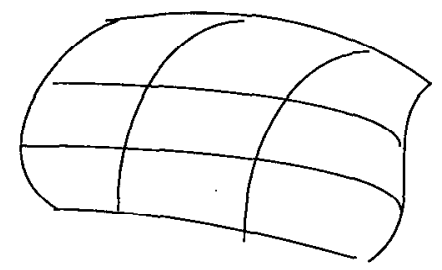

There are two ways to choose the coordinate transformation to connect the logical 
with the physical space. The first is to choose an algebraic relationship, such as a polynomial or a trigonometric function. This is easiest in one dimension, and is often used for boundaries. The second is to choose a PDE to represent the relationship between logical and physical space.

Elliptic PDE's work best because of their properties:

1. The solutions are smooth (have continuous functions and derivatives) if the coefficients are smooth.

2. The solutions are determined by the values on the boundary. (The grid point distribution on the boundary must be chosen before the PDE is solved on the interior grid points. The algebraic method is often used for the 1 dimensional boundary to a 2 dimensional problem. Either method can be used in the 3 dimensional case).

3. The maximum and minimum of the solution will occur on the boundaries.

In choosing which elliptic equation to use, there are three important considerations:

1. Each point in each space must map to a point which is within the other space.

2. The transformation must be "one to one". That is, each point must map into one and only one point.

3. The transformation must be "onto". That is, if one point in one space maps to another point in the other space, the reverse transformation must map back to the first point.

This makes the transformation isomorphic.

The Jacobian matrix is an important tool in coordinate transformations. For the mapping from physical to logical space, the Jacobian matrix is:

$$
\left[\begin{array}{l}
\frac{\partial x}{\partial \xi} \frac{\partial x}{\partial \eta} \\
\frac{\partial y}{\partial \xi} \frac{\partial y}{\partial \eta}
\end{array}\right]
$$

The determinant of this matrix, $\mathrm{J}$, represents the volume of a mesh zone in the physi- 
cal space. The "inverse mapping" theorem [17] states that the transformation is one to one if and only if $\mathrm{J}$ is non-zero.

Consider Laplace's equation in a two dimensional example. There are two possible mappings between the logical and physical space using this equation:

$$
\text { 1. } \frac{\partial^{2} \xi}{\partial x^{2}}+\frac{\partial^{2} \xi}{\partial y^{2}}=0 \text { and } \frac{\partial^{2} \eta}{\partial x^{2}}+\frac{\partial^{2} \eta}{\partial y^{2}}=0
$$

or

$$
\text { 2. } \frac{\partial^{2} x}{\partial \xi^{2}}+\frac{\partial^{2} x}{\partial \eta^{2}}=0 \text { and } \frac{\partial^{2} y}{\partial \xi^{2}}+\frac{\partial^{2} y}{\partial \eta^{2}}=0
$$

The second mapping looks more useful, since the object is to find $(\mathrm{x}, \mathrm{y})$. The logical grid $(\xi, \eta)$ is fixed, and is used to generate a new physical mesh at each remap step of the ALE code. The second mapping also involves easier math because $(\xi, \eta)$ are orthogonal and evenly spaced, so the derivatives are simple finite difference approximations.

However, it has been shown [17] that the second mapping gives $J=0$ if the physical region is not convex. So the first Laplace's equation mapping, known as the equipotential method, is used instead. This means the transformation from logical to physical space must also be found in order to find $x$ and $y$ in terms of $(\xi, \eta)$.

In order to perform finite difference approximations for derivatives in $(x, y)$ space, the nearest neighbors of each grid point need to be well defined. In cases where they are not, calculus of variations can be used to change the differential equation into an integral one. 
The equipotential method is a simple PDE griding technique, with the advantage of guaranteed "one to one." But it has the disadvantage of spreading mesh points evenly across the domain. For example, if extra mesh points have been clustered in one part of the domain to describe an interface, the equipotential method will spread them out so that they are more evenly distributed across the domain. The equipotential method also tends to cluster grid lines over convex boundaries, and spread them out over concave boundaries.

An ALE code also wants to create each new mesh in response to the condition of the flow in the most recent time step. Grid points should move only if they are becoming so tangled that the program is imperiled. Some regions may behave in a pure Eulerian fashion, with no grid motion, while others follow the flow in a completely Lagrangian fashion. Still other points may move at some pace in between, determined by the needs of the flow.

The most recent grid needs to determine how the new grid will be generated. There are two ways that Laplace's equation can be modified to allow control of the node spacing. One is to use Poisson's equation instead of Laplace's:

$$
\frac{\partial^{2} \xi}{\partial x^{2}}+\frac{\partial^{2} \xi}{\partial y^{2}}=F(x, y) \text { and } \frac{\partial^{2} \eta}{\partial x^{2}}+\frac{\partial^{2} \eta}{\partial y^{2}}=G(x, y)
$$

But it is harder to have a selective influence considering local conditions with Poisson's equation. $F(x, y)$ and $G(x, y)$ act as source terms in a heat conduction type equation. They make hot parts hotter.

A better way to modify the equipotential method is to use a weighted Laplace's equation: 


$$
\begin{aligned}
& \frac{\partial}{\partial x}\left(W_{1}(x, y) \cdot \frac{\partial \xi}{\partial x}\right)+\frac{\partial}{\partial y}\left(W_{1}(x, y) \cdot \frac{\partial \xi}{\partial y}\right)=0 \\
& \frac{\partial}{\partial x}\left(W_{2}(x, y) \cdot \frac{\partial \eta}{\partial x}\right)+\frac{\partial}{\partial y}\left(W_{2}(x, y) \cdot \frac{\partial \eta}{\partial y}\right)=0
\end{aligned}
$$

As with the heat example, diffusion coefficients cause the heat to move from hot to cold, creating an even continuum between extremes. Information from the most recent grid can be used in calculating $W_{1}$ and $W_{2}$, allowing the mesh to respond to the most recent mesh motion. The Jacobian J, representing the zonal volume, can be used, as well as physical quantities such as pressure. The solution to this equation is taken iteratively [63]. Once the new grid is defined, density, momentum and energy are redistributed on the new mesh with an advection algorithm. 


\section{References}

1. U. Alon, J. Hecht, D. Ofer, and D. Shvarts, Power Laws and Similarity of RayleighTaylor and Richtmyer-Meshkov Mixing Fronts at All Density Ratios, Physical Review Letters, vol 74 no 4, 23 Jan 1995

2. Paul A.K. Amala, Large-Eddy Simulation of the Rayleigh-Taylor Instability on a Massively Parallel Computer, Ph.D. Thesis, UC Davis, Davis CA, 1995

3. R.T. Barton, Development of a Multimaterial Two-Dimensional, Arbitrary Lagrangian-Eulerian Mesh Computer Program, in J.M. Centrella, J.M. LeBlanc, and R.L. Bowers, Numerical Astrophysics, Jones and Bartlett Publishers, Inc. Boston 1985

4. K.S. Budil et al., Experimental Comparison of Classical versus Ablative RayleighTaylor Instability, Physical Review Letters, vol. 76 no. 24, 10 June 1996

5. K.S. Budil et al., A novel method for diagnosing the growth of subresolution-scale perturbations, Rev. Sci. Instrum., vol 68 no 1, January 1997

6. E.M. Campbell, Recent results from the Nova program at LLNL, Laser and Particle Beams, vol 9 no 2, p. 209, 1991

7. V.M. Canuto, Large eddy sirnulation of turbulence: a subgrid scale model including shear, vorticity, rotation and buoyancy, The Astrophysical Journal, 428, p. 729-752, June 201994

8. E.J. Caramana and M.J. Shaskov, Elimination of artificial grid distortion and hourglass-type motions by means of Lagrangian subzonal masses and pressures, Journal of Computational Physics, 142, p. 521-561, 1998

9. S. Chandrasckhar, Hydrodynamic and Hydromagnetic Stability, Dover Publications, New York, 1961

10. Y.C. Chang, T. Y. Hou, B. Merriman and S. Osher, A Level set Formulation of Eulerian Interface Capturing Methods for Incompressible Flows, Journal of Computational Physics, 124, p.449-464, 1996

11. C. Cherfils and K.O. Mikaelian, Simple model for the turbulent mixing width at an ablating surface, Physics of Fluids, vol 8 no 2, Feb 1996

12. Lawrence D. Cloutman, The LUVD11 Large Eddy Simulation Model, Lawrence Livermore National Laboratory, internal report UCRL-107128, 1991

13. R.D. Cook, D.S. Malkus and M.E. Plesha, Concepts and Applications of Finite Element Analysis, John Wiley \& Sons, 1989

14. S. Corrsin, Limitations of gradient transport models in random walks and in turbulence, in F.N. Frenkiel and R.E. Munn, editors, Trubulent Diffusion in Environmental Pollution, Academic Press, New York, 1974

15. G. Dimonte and M. Schneider, Density ratio dependence of Rayleigh-Taylor mixing for sustained and impulsive acceleration histories, (unpublished) 1998 
16. Guy Dimonte, Nonlinear evolution of the Rayleigh-Taylor and Richtmyer-Meshkov instabilities, Physics of Plasmas, vol 6 no 5, May 1999

17. Eveline I. Dube, Diffusive Mesh Relaxation in ALE Finite Element Numerical Simulations, Ph.D. Thesis, UC Davis, Davis CA, 1996

18. J.H. Ferziger, Higher-level Simulations of Turbulent Flows, in J.A. Essers (editor) Computational Methods for Turbulent. Transonic, and Viscous Flows, Hemisphere Publishing Corp., New York, 1983

19. C.A.J. Fletcher, Computational Techniques for Fluid Dynamics Vol. 1, second edition, Springer-Verlag, New York, 1991

20. R.M. Frank and R.B. Lazarus, Mixed Eulerian-Lagrangian Method in B. Alder et al. (editors), Methods in Computational Physics Vol 3, Fundamental Methods in Hydrodynamics, Academic Press, San Francisco, 1964

21. N. Freed, D. Ofer, D. Shvarts, and S. Orsag, Two-phase flow analysis of self-similar turbulent mixing by Rayleigh-Taylor instability, Physics of Fluids A, vol 3 no 5, May 1991

22. C. Fureby et al., A comparative study of subgrid scale models in homogeneous isotropic turbulence, Physics of Fluids, vol 9 no 5, May 1997

23. C.L. Gardner, J. Glimm, O. McBryan, R. Menikoff, D.H. Sharp, and Q. Zhang, The Dynamics of bubble growth for Rayleigh-Taylor unstable interfaces, Physics of Fluids, vol 31 no 3, March 1988

24. Curtis F. Gerald and Patric O. Wheatly, Applied Numerical Analysis, fourth edition, Addison-Wesley Publishing Co., New York, 1989

25. M. Germano, U. Piomelli, P. Moin, and W.H. Cabot, A dynamic subgrid-scale eddy viscosity model, Physics of Fluids, vol 3 no 7, p. 1760-1765, July 1991

26. S. Ghosal and P. Moin, The Basic Equations for the Large Eddy Simulation of Turbulent Flows in Complex Geometry, Journal of Computational Physics, 118, p. 24-37, 1995

27. J. Glimm, X.L. Li, R. Menikoff, D. Sharp, and Q.A. Zhang, Numerical study of bubble interactions in Rayleigh-Taylor instability for compressible fluids, Physics of Fluids A, vol 2 no 11, Nov 1990

28. J. Glimm, Q. Zhang, and D.H. Sharp, The renormalization group dynamics of chaotic mixing of unstable interfaces, Physics of Fluids A, vol 3 no 5, May 1991

29. J. Glimm, J. Grove, X. Li, K. Shyue. Y. Zeng and Q. Zhang, Three-dimensional Front Tracking, SIAM J. Sci. Comput., vol 19 no 3, p. 703-727, May 1998

30. S.W. Haan, Onset of nonlinear saturation for Rayleigh-Taylor growth in the presence of a full spectrum of modes, Physical Review A, vol 39 no 11, June 11989

31. J. Hecht, D. Ofer, U. Alon, D. Shvarts, S.A. Orsag, and R.L. McCory, Three-Dimensional simulations and analysis of the nonlinear stage of the Rayeligh-Taylor instability, Laser and Particle Beams, vol 13 no 3, p 423-440, 1995 
32. Charles Hirsch, Numerical Computation of Internal and External Flows Vol 1: Fundamentals of Numerical Discretization , John Wiley \& Sons, New York, 1988

33. C.W. Hirt, A.A. Amsden, and J.L. Cook, An Arbitrary Lagrangian-Eulerian Computing Method for All Flow Speeds, Journal of Computational Physics, 14, p. 227253, 1974

34. Joe D. Hoffman, Numerical Methods for Engineers and Scientists, McGraw Hill, San Francisco, 1992

35. T.J. Hughes, I. Levit and J. Winget, An element-by-element algorithm for problems of structural and solid mechanics, Comp Meth. in Appl. mech. and Eng., 36, p. 241254,1983

36. O.P. Jacquotte and T.J. Oden, Analysis of hourglass instabilities and control of underintgrated finite element methods, Comp. Meth. in App. Mech. \& Eng., 44, p. 339-363, 1984

37. Richard Johnsonbaugh and Martin Kalin, Applications Programming in ANSI C, second edition, Macmillan Publishing Company, New York, 1993

38. J. Kane et al., Supernova-relevant hydrodynamic instability experiments on the NOVA laser, The Astrophysical Journal, 478, p. L75-L78, April 11997

39. J.D. Kilkenny et al., A review of the ablative stabilization of the Rayleigh-Taylor instability in regimes relevant to inertial confinement fusion, Physics of Plasmas, vol 1 no 5, p. 1379-1389, May 1994

40. C.E. Leith, Stochastic Backscatter Formulation for Three-Dimensional Compressible Flow, in B. Galerpin and S.A. Orszag (editors), Large Eddy Simulation of Complex Engineering and Geophysical Flows, Cambridge University Press, New York, 1993

41. Marcel Lesieur, Turbulence in Fluids, Martinus Nijhoff Publishers, Boston, 1987

42. D.C. Leslie, Simulation Methods for Turbulent Flows, in K.W. Morton and M.J. Baines, Numerical Methods for Fluid Dynamics, Academic Press, New York, 1982

43. Randall J. LeVeque, Numerical Methods for Conservation Laws, second edition, Birkhauser Verlag, Boston, 1992

44. X.L. Li, Study of three-dimensional Rayleigh-Taylor Instability in compressible fluids through level set method and parallel computation, Physics of Fluids A, vol 5 no 8, Aug 1993

45. X.L. Li, B.X. Jin, and J. Glimm, Numerical Study for the Three-Dimensional Rayleigh-Taylor Instability through the TVD/AC Scheme and Parallel Compuatation, Journal of Computational Physics, 126, p. 343-355, 1996

46. P.F. Linden, J.M. Redondo, and D.L. Youngs, Molecular Mixing in Rayleigh-Taylor Instability, Journal of Fluid Mechanics, vol 256, p. 97-124, 1994

47. P.J. Mason and D.J. Thompson, Stochastic backscatter in large-eddy simulations of boundary layers, Journal of Fluid Mechanics, vol 242, p. 51-78, 1992 
48. P.J. Mason, Large-eddy simulation: A critical review of the technique, Q.J.R. Meteorological Society, 120, p. 1-26, 1994

49. R.C. McCallen, Large-Eddy Simulation of Turbulent Flow Using the Finite Element Method, Ph.D. Thesis, UC Davis, Davis CA, 1993

50. P. Moin and J. Kim, Numerical investigation of turbulent channel flow, Journal of Fluid Mechanics, vol 118, p. 341-377, 1982

51. W.F. Noh, CEL: a Time-Dependant, Two-Space_Dimensional, Coupled EulerianLagrange Code, in B. Alder et al. (editors), Methods in Computational Physics Vol 3, Fundamental Methods in Hydrodynamics, Academic Press, San Francisco, 1964

52. D. Ofer, D. Shvarts, Z. Zinamon, and S. Orszag, Mode coupling in nonlinear Rayleigh-Taylor instability, Phys. Fluids B, vol 4 no 11, p. 3549-3561, Nov 1992

53. W.H. Press et al., Numerical Recipes in C, second edition, Cambridge University Press, New York, 1992, p. 282

54. K.I. Read, Experimental Investigation of Turbulent Mixing by Rayleigh-Taylor Instability, Physica D, 12, p. 45-58, 1984

55. P.L. Roe, Some contributions to the modeling of discontinuous flows, Lecture Notes in Applied Mathematics, 22, p. 163-193, 1985

56. R. Richtmyer and K.W. Morton, in Difference Methods for Intial Value Problems, Interscience, New York, 1957

57. M.B. Schneider, G. Dimonte, and B. Remington, Large and Small Scale Structure in Rayleigh-Taylor Mixing, Physical Review Letters, vol 80 no 16, April 201998

58. U. Schumann, Stochastic backscatter of turbulent energy and scalar variance from random subgrid-scale fluxes, submitted to Physics of Fluids A, December 1992

59. D.H. Sharp, An overview of Rayleigh-Taylor instability, Physica 12 D, p. 3-18, 1984

60. J. Smagorinsky, General circulation experiments with the primitive equations I. The basic experiment, Monthly Weather Review, vol 91 no 3, p. 99-166, 1963

61. L. Smarr, J.R. Wilson, R.T. Barton, and R.L. Bowers, Rayleigh-Taylor overturn in supernova core collapse, The Astrophysical Journal, 246, p.515-525, June 11981

62. Gilbert Strang, On the construction and comparison of difference schemes, SIAM J. Numer. Anal., vol. 5 no. 3, Sept 1968

63. Robert E. Tipton, Grid Optimization by Equipotential Relaxation, Lawrence Livermore National Laboratory, unpublished, 1992

64. Robert E. Tipton, The Eulerian Interface Advection Scheme in CALE, Lawrence Livermore National Laboratory, unpublished, 1994

65. H. Tennekes and J.L. Lumley, A First Course in Turbulence, The MIT Press, Cambridge MA, 1972

66. Eleuterio F. Toro, Rieman Solvers and Numerical Methods for Fluid Dynamics, Springer, New York, 1997 
67. Bram Van Leer, Towards the Ultimate Conservative Difference Scheme. IV A New Approach to Numerical Convection, Journal of Computational Physics, 23, p. 276288, 1977

68. Frank M. White, Viscous Fluid Flow, second edition, McGraw-Hill, San Francisco, 1991

69. Mark L. Wilkins, Calculation of Elastic-Plastic Flow, in B. Alder et al. (editors), Methods in Computational Physics Vol 3, Fundamental Methods in Hydrodynamics, Academic Press, San Francisco, 1964

70. D.A. Young and E.M. Corey, A new global equation of state model for hot, dense matter, J. Appl. Phys., vol 78 no 6, 15 Sept 1995

71. D.L. Youngs, Time Dependant Multi-Matreial Flow with Large Fluid Distortion, in K.W. Morton and M.J. Baines, Numerical Methods for Fluid Dynamics, Academic Press, New York, 1982

72. D.L. Youngs, Numerical Simulation of Turbuelnt Mixing by Rayleigh-Taylor Instability, Physica D, 12, p. 32-44, 1984

73. D.L. Youngs, Modeling turbulent mixing by Rayleigh-Taylor instability, Physica D, vol 37, p. 270-287, 1989

74. D.L. Youngs, Three-dimensional numerical simulation of turbulent mixing by Rayleigh-Taylor instability, Phys. Fluids A, vol 2 no 5, p. 1312-1319, May 1991.

75. D.L. Youngs, Numerical Simulation of Mixing by Rayleigh-Taylor and RichtmyerMeshkov Instabilities, Laser and Particle Beams, vol 12, no 4, p 725-750, 1994

76. Ya. B. Zel'dovich and Yu. P. Raizer, Physics of Shock Waves and High-Temperature Hydrodynamic Phenomena (Vol 1), Academic Press, New York, 1966 
